\title{
Corner defects in almost planar interface propagation
}

\section{Défauts faibles en propagation d'interfaces planes}

\author{
Mariana Haragus ${ }^{\mathrm{a}}$, Arnd Scheel ${ }^{\mathrm{b}, *}$ \\ a Université de Franche-Comté, Département de Mathématiques, 16, route de Gray, 25030 Besançon cedex, France \\ ${ }^{\mathrm{b}}$ University of Minnesota, School of Mathematics, 206 Church St. S.E., Minneapolis, MN 55455, USA
}

Received 21 June 2003; accepted 18 March 2005

Available online 14 July 2005

\begin{abstract}
We study existence and stability of interfaces in reaction-diffusion systems which are asymptotically planar. The problem of existence of corners is reduced to an ordinary differential equation that can be viewed as the travelling-wave equation to a viscous conservation law or variants of the Kuramoto-Sivashinsky equation. The corner typically, but not always, points in the direction opposite to the direction of propagation. For the existence and stability problem, we rely on a spatial dynamics formulation with an appropriate equivariant parameterization for relative equilibria.
\end{abstract}

(c) 2006 L'Association Publications de l'Institut Henri Poincaré. Published by Elsevier B.V. All rights reserved

\section{Résumé}

Nous étudions l'existence et la stabilité des interfaces asymptotiquement planes dans des systèmes de réaction-diffusion. Le problème de l'existence des défauts est réduit à l'étude d'une équation différentielle ordinaire qui est, selon le cas, approchée par l'équation stationnaire d'une loi de conservation scalaire ou d'une variante de l'équation de Kuramoto-Sivashinsky. Typiquement, les défauts pointent dans la direction opposée à la direction de propagation. Pour l'analyse des problèmes d'existence et de stabilité, nous utilisons une formulation de type dynamique spatiale combinée avec une paramétrisation adéquate d'équilibres relatifs.

(C) 2006 L'Association Publications de l'Institut Henri Poincaré. Published by Elsevier B.V. All rights reserved

Keywords: Interfaces; Stability; Reaction-diffusion systems; Burgers equation; Kuramoto-Sivashinsky equation; Quadratic systems

\footnotetext{
* Corresponding author.

E-mail address: scheel@math.umn.edu (A. Scheel).
} 


\section{Introduction}

Characterizing propagation of interfaces in spatially extended systems is a major challenge in the applied sciences. Flame fronts in solid and gaseous combustion have stimulated a variety of different approaches to interface formation and propagation $[17,44,64]$. Experimental observation and theoretical predictions range from rigid plane front propagation over periodically oscillating speeds and cellular patterns on the interface, to seemingly chaotic motion of the interface. In a slightly different context, front and pulse propagation turns out to be crucial for the dynamics of many self-organizing chemical reactions, such as the carbon-monoxide oxidation on platinum surfaces [30] or the Belousov-Zhabotinsky reaction [66]. One-dimensional interfaces have been observed in spiral wave patterns, where interfacial corners naturally arise at the domain boundaries between different spiral cores [66]. More recently, oscillatory front propagation [34] and interfaces between homogeneous and patterned states [22] have been studied in the Belousov-Zhabotinsky reaction. Propagation and reflective or annihilation collision of 2-dimensional pulse trains has also been observed in the CO-oxidation [25]. In the CIMA reaction, famous for exhibiting stationary Turing patterns, propagation and propagation failure of a one-dimensional interface separating a region occupied by a hexagonal lattice built with isolated Turing spots, into an unpatterned region governs the later stage of spot replication; see [9] for an experimental survey and [46] for (mostly one-dimensional) theoretical approaches. Interfaces between patterned states arise in many other applied areas. We mention semiconductors [58], viscous shock waves [41], Rayleigh-Bénard convection [48] or certain models for extended lasers [40].

In a singular perturbation approach to interface propagation, spatial variables are scaled such that the interface becomes a sharp line, for which a geometric evolution equation can be derived from inner and outer expansions at the interfacial region; see $[15,61]$ for a variety of applications of this method. In many cases, the formal asymptotics can be justified, either in a general dynamical setup [6], or in specific contexts [29]. The most general results are available when a comparison principle is at hand [2].

More recently, interface propagation has been addressed from a different perspective. The common feature is that existence and stability are considered in unbounded domains, corresponding to the inner expansion in the sharp interface limit. For various reasons, however, a scaling cannot be rigorously justified such that interfaces have to be studied in the original equations. We mention recent work on propagation of fronts in discrete two-dimensional lattices with possible pinning of interfaces [7], stability of plane viscous shocks [28,36], and existence and stability of conically shaped fronts in scalar reaction-diffusion models for combustion [3,20,21].

We refer the reader to the beautiful review [63] as a guide to the tremendous amount of work on (mostly onedimensional) front propagation in the physics literature.

In this article, we focus on existence and stability of almost planar interfaces. Almost planar here refers to the angle of the interface at each point, relative to a fixed planar interface. Most of the interfaces that we construct will be planar at infinity, with possibly different orientations at $+\infty$ and $-\infty$ in an arclength parameterization. We refer to all these types of interfaces as corner defects. Different angles at $\pm \infty$ result in conically shaped interfaces, like for example the travelling waves constructed in [20]. In an isotropic sharp interface scaling, conical interfaces correspond to corners. Equal angles at $\pm \infty$ may result in infinitesimal step discontinuities, when the position of the interface differs at $\pm \infty$. We construct corner defects as perturbations of a planar interface. Assumptions are solely on the existence of a primary planar travelling-wave solution and spectral properties of the linearization at the planar wave. All interfaces that we construct in the present article are stationary or time-periodic patterns in an appropriately comoving frame. However, we give stability results which show that "open" classes of initial conditions actually converge to the corner-shaped interfaces we constructed before. The results are stated for reaction-diffusion systems but the method is sufficiently general to cover most applications mentioned above. In particular we do not rely on monotonicity arguments or comparison principles such that we can naturally include the case of interfaces separating patterned states from spatially homogeneous states.

The method we use is based on the (essentially one-dimensional) dynamical systems approach to the existence of bounded solutions to elliptic equations in cylinders introduced by Kirchgässner [39]. Later this approach has been used to construct nontrivial transverse modulations of one-dimensional waves, such as pulses or periodic 
solutions, a phenomenon the authors referred to as dimension breaking [23,24]. The main idea is to consider an elliptic equation, posed on the $(x, y)$-plane in a neighborhood of an $x$-independent wave $q_{*}(y)$ as a dynamical system in the $x$-variable and rely on dynamical systems tools such as center-manifold reduction and bifurcation theory to construct bounded solutions to the elliptic equation in a neighborhood of the original wave. Nontrivial, that is nonequilibrium, $x$-"dynamics" then correspond to nontrivial $x$-profiles.

In the present work, we extend these ideas, incorporating the shift of the $y$-profile $q_{*}(y)$ into the reduced dynamics. We then respect this affine action of the symmetry group in the construction and parameterization of the center-manifold such that the reduced equations take a skew-product form. The concept of an equivariant reduction and skew-product description of bifurcations in the presence of noncompact, nonsmooth group actions has been introduced in [14,55] in order to describe meandering and drift motion of spiral waves under the presence of the Euclidean group of rotations and translations in the plane. The construction of the center-manifold is "semi-global" in the sense that a neighborhood of all translates of the primary solution is described by the reduced equations. For example, constant drift $\xi^{\prime}=\eta$ in $x$-dynamics along the translates $q_{*}(y+\xi)$, corresponds to the original front inclined by an angle $\vartheta=\arctan \eta$.

The methods and results are related to recent work in [11,54] on dynamics of defects in oscillatory media. The common feature between the present work and the study of wave trains is the presence of a neutral eigenvalue induced by the translation of a primary profile, the Goldstone mode. A major difference lies in the fact that wave trains possess a noncompact isotropy generated by translations of one period, such that the resulting symmetry action is isomorphic to the circle group, whereas in our case, the isotropy of the travelling wave is trivial. As a consequence, we have to study bifurcations from a noncompact group orbit, isomorphic to $\mathbb{R}$, whereas the group orbit in $[11,54]$ is compact, a circle.

We develop the idea, prove a reduction theorem, and describe the most basic shock-type corner solutions in Section 2. We prove asymptotic stability of these structures in Section 3. We then consider more complicated scenarios, where the front undergoes a transverse long-wavelength instability, in Section 4, and when the primary front is pulsating, in Section 5. We conclude with a discussion, pointing out possible generalizations and open questions in Section 6.

\section{Existence of corners}

We introduce the general setup for travelling waves in reaction-diffusion systems and define the typical types of corners one might expect to find. We then state and prove the first main result on existence and nonexistence of corner defects for interfaces separating two homogeneous states. We conclude the section with several examples and possible extensions.

Throughout the paper, we consider the reaction-diffusion system

$$
u_{t}=D \Delta_{x, y} u+c \partial_{y} u+f(u),
$$

where $u \in \mathbb{R}^{N}$ is a vector of $N$ chemical species, $D=\operatorname{diag}\left(D_{1}, \ldots, D_{N}\right)>0$ is a positive, diagonal diffusion matrix, and $(x, y) \in \mathbb{R}^{n} \times \mathbb{R}$. The reaction kinetics $f$ are assumed to be smooth. The Laplacian is assumed to be isotropic $\Delta_{x, y}=\Delta_{x}+\partial_{y y}$. The speed $c>0$ is assumed to be positive, such that bounded solutions to the stationary equation

$$
D \Delta_{x, y} u+c \partial_{y} u+f(u)=0,
$$

are (right-)travelling-wave solutions $u(x, y-c t)$ of the reaction-diffusion system in the steady frame

$$
u_{t}=D \Delta_{x, y} u+f(u),
$$

with direction of propagation in the positive $y$-direction. We will assume $n=1$ such that $(x, y) \in \mathbb{R}^{2}$, throughout. We briefly comment on higher space dimensions in Section 6. 
Throughout this section, we will assume existence of a planar travelling wave connecting two homogeneous equilibria.

Hypothesis 2.1 (Existence). We assume that there exists $c_{*}>0$ and asymptotic states $q_{ \pm}$such that there exists an $x$-independent planar travelling-wave solution $q_{*}(y)$ of (2.2)

$$
D q_{*}^{\prime \prime}+c_{*} q_{*}^{\prime}+f\left(q_{*}\right)=0,
$$

connecting $q_{-}$and $q_{+}$, i.e.

$$
q_{*}(y) \rightarrow q_{+} \quad \text { for } y \rightarrow+\infty, \quad q_{*}(y) \rightarrow q_{-} \text {for } y \rightarrow-\infty .
$$

We emphasize that we allow for the possibility of pulses, $q_{+}=q_{-}$.

The second assumption in this section is concerned with stability of the above travelling wave solution. Therefore, consider the linearized operator

$$
\mathcal{L}_{*}: H^{2}\left(\mathbb{R}, \mathbb{R}^{N}\right) \subset L^{2}\left(\mathbb{R}, \mathbb{R}^{N}\right) \rightarrow L^{2}\left(\mathbb{R}, \mathbb{R}^{N}\right), \quad u \mapsto-\partial_{y y} u-D^{-1}\left(c_{*} \partial_{y} u+f^{\prime}\left(q_{*}(\cdot)\right) u\right) .
$$

Notice that under suitable decay assumptions, $q_{*}^{\prime}$ belongs to the kernel of $\mathcal{L}_{*}$ due to the translation invariance in $y$.

Hypothesis 2.2 (Zero-stability). We assume that $\mathcal{L}_{*}-\lambda$ id is invertible for all $\lambda<0$ and that $\lambda=0$ is an isolated eigenvalue with algebraic multiplicity one.

Although this might not seem obvious, Hypothesis 2.2 is intimately related to stability properties of the travelling wave $q_{*}(\cdot)$. Consider the linearized operator

$$
\mathcal{M}_{*}: H^{2}\left(\mathbb{R}^{2}, \mathbb{R}^{N}\right) \subset L^{2}\left(\mathbb{R}^{2}, \mathbb{R}^{N}\right) \rightarrow L^{2}\left(\mathbb{R}^{2}, \mathbb{R}^{N}\right), \quad u \mapsto D \Delta_{x, y} u+c_{*} \partial_{y} u+f^{\prime}\left(q_{*}(\cdot)\right) u,
$$

and its Fourier conjugates

$$
\mathcal{M}_{k}: H^{2}\left(\mathbb{R}, \mathbb{R}^{N}\right) \subset L^{2}\left(\mathbb{R}, \mathbb{R}^{N}\right) \rightarrow L^{2}\left(\mathbb{R}, \mathbb{R}^{N}\right), \quad u \mapsto D\left(\partial_{y y}-k^{2}\right) u+c_{*} \partial_{y} u+f^{\prime}\left(q_{*}(\cdot)\right) u .
$$

Hypothesis 2.3 (Transverse asymptotic stability). Assume that the travelling wave is asymptotically stable in one space dimension, that is, the essential spectrum of $\mathcal{M}_{0}$ is strictly contained in the left complex half plane and zero is the only eigenvalue in the closed right half plane, with algebraic multiplicity one. Furthermore, assume that the spectra of $\mathcal{M}_{k}$, for $k \neq 0$ are strictly contained in the left half plane and that the unique eigenvalue $\lambda_{\mathrm{d}}(k), k \sim 0$ with $\lambda_{d}(0)=\lambda_{d}^{\prime}(0)=0$ satisfies $\lambda_{d}^{\prime \prime}(0)<0$; see Fig. 1 .

\section{Remark 2.4.}

(i) This hypothesis and, in particular, the quadratic tangency of the dispersion relation $\lambda_{d}^{\prime \prime}(0)<0$ implies asymptotic stability of the travelling wave with respect to perturbations that are sufficiently localized in the transverse, $x$-direction $[28,35,36]$.
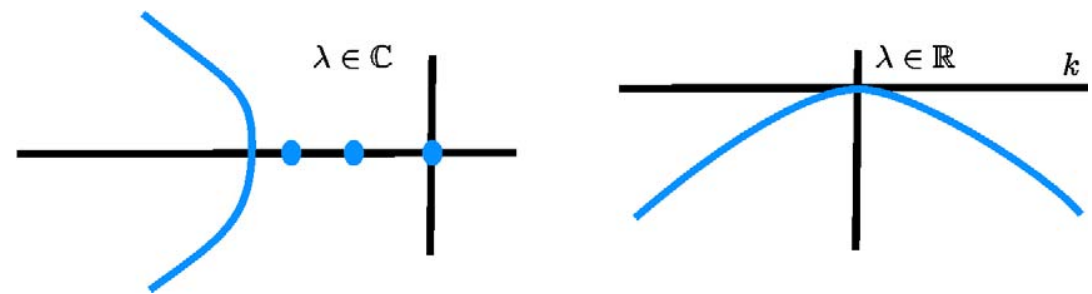

Fig. 1. To the left the spectrum of $\mathcal{M}_{0}$ and to the right the critical spectra of the $\mathcal{M}_{k}$ parameterized by $k$. 
(ii) For equal diffusion constants, $D=d_{1}$ id, the second part of Hypothesis 2.3 is a consequence of the first part, on the spectrum of $\mathcal{M}_{0}$. However, this is not always the case when the diffusion constants are not equal; see also Section 4.

Lemma 2.5. Hypothesis 2.3 implies that Hypothesis 2.2 on stability holds.

Proof. The proof is similar to [57, Lemma 2.11, Remark 2.12]. Since $\mathcal{M}_{k}$ is invertible for all $k \neq 0$, we immediately conclude, upon multiplying by the inverse of the diffusion matrix $D$, that $\mathcal{L}_{*}+k^{2}$ is invertible for all $k \neq 0$. Similarly, the kernels of $\mathcal{M}_{0}$ and $\mathcal{L}_{*}$ coincide. Denote by $u(k)$ the (normalized) unique family of eigenvectors to the eigenvalue $\lambda_{\mathrm{d}}(k)$ of $\mathcal{M}_{k}$,

$$
D\left(\partial_{y y}-k^{2}\right) u(k)+c_{*} \partial_{y} u(k)+f^{\prime}\left(q_{*}(\cdot)\right) u(k)=\lambda_{\mathrm{d}}(k) u(k) .
$$

Differentiating this equality twice, and evaluating in $k=0$ with $u(0)=q_{*}^{\prime}, u^{\prime}(0)=0, \lambda_{\mathrm{d}}(0)=0$, and $\lambda_{\mathrm{d}}^{\prime}(0)=0$, we find

$$
D \partial_{y y} u^{\prime \prime}(0)+c_{*} \partial_{y} u^{\prime \prime}(0)+f^{\prime}\left(q_{*}(\cdot)\right) u^{\prime \prime}(0)=\lambda_{\mathrm{d}}^{\prime \prime}(0) q_{*}^{\prime}+2 D q_{*}^{\prime},
$$

where $\lambda_{d}^{\prime \prime}(0)$ is the unique Lagrange multiplier such that (2.9) possesses a nontrivial solution. A vector $\tilde{u}$ in the generalized kernel of $\mathcal{L}_{*}$ solves

$$
\partial_{y y} \tilde{u}+D^{-1}\left(c_{*} \partial_{y} \tilde{u}+f^{\prime}\left(q_{*}(\cdot)\right) \tilde{u}\right)=q_{*}^{\prime} .
$$

Upon comparing (2.10) and (2.9), where $\lambda_{\mathrm{d}}^{\prime \prime}(0) \neq 0$, we conclude that (2.10) does not possess a solution and $\lambda=0$ is algebraically simple as stated in Hypothesis 2.2 .

\section{Remark 2.6.}

(i) The converse implication generally fails. For example, after a temporal Hopf bifurcation of a one-dimensional propagating front, caused by point spectrum crossing the imaginary axis, Hypothesis 2.2 would still hold, whereas Hypothesis 2.3 would fail. However, Hypothesis 2.2 does imply the quadratic tangency of the dispersion relation $\lambda_{d}^{\prime \prime}(0)<0$. This fact will be used later in Theorem 1 .

(ii) It is straightforward to verify that in the case of a fourth order tangency $\lambda_{\mathrm{d}}(k) \sim-\left(\lambda_{\mathrm{d}}^{(4)}(0) / 4\right.$ !) $k^{4}, \lambda=0$ is algebraically double as an eigenvalue of $\mathcal{L}_{*}$; see [57, Remark 2.12]. This fact will become relevant in Section 4.2.

The following classification of corner defects is much inspired by the classification of defects in oscillatory media; see [54].

Definition 2.7 (Corner defects). A solution $u(x, y)$ of the travelling-wave problem is called an almost planar travelling-wave solution $\delta$-close to $q_{*}$, if $u$ is of the form

$$
u(x, y)=q_{*}(y+\xi(x))+w(x, y),
$$

with $\xi \in C^{2}(\mathbb{R})$ and

$$
\sup _{x}\left|\xi^{\prime}(x)\right|<\delta, \quad \sup _{x}|w(x, \cdot)|_{H^{1}\left(\mathbb{R}, \mathbb{R}^{N}\right)}<\delta, \quad\left|c-c_{*}\right|<\delta .
$$

We say that $u$ is trivial if $u$ is a rotated planar interface $u=q_{*}((\cos \vartheta) x+(\sin \vartheta) y)$, for some $\vartheta \in \mathbb{R}$.

We say that $u$ is a corner defect if it is of the form (2.11) and $\xi^{\prime}(x) \rightarrow \eta_{ \pm} \in \mathbb{R}$, as $x \rightarrow \pm \infty$. We distinguish corner defects according to the following list: 


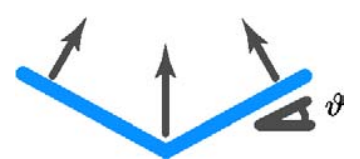

(a)

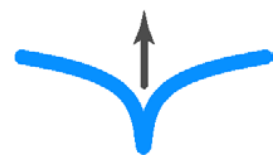

(b)

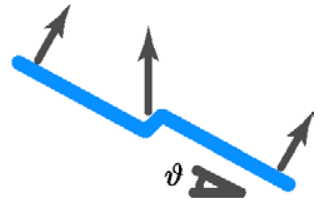

(c)

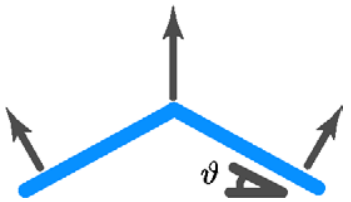

(d)

Fig. 2. Schematic plot of the four different types of corner defects, interior corner (a), hole (b), step (c), and exterior corner (d). The middle arrows indicate the speed of the defect, whereas the left and right (smaller) arrows indicate the normal speed of propagation of the interface. The angle $\vartheta$ is given by $\tan \vartheta=\eta_{+}$.

- if $\eta_{+}<\eta_{-}$we say that the corner defect is an interior corner;

- if $\eta_{+}=\eta_{-}=0$ we say that the corner defect is a hole;

- if $\eta_{+}=\eta_{-} \neq 0$ we say that the corner defect is a step;

- if $\eta_{+}>\eta_{-}$we say that the corner defect is an exterior corner;

see Fig. 2.

Remark 2.8. If we think of individual points on the interface evolving with the normal speed $c_{*}$, we notice that interface is consumed on both sides of interior corners and interface is generated at exterior corners. At a step, interface is consumed on one side and generated on the other side, whereas at a hole, interfacial points neither enter nor leave the defect at leading order. In addition to the geometric characterization in Definition 2.7 we therefore suggest the following dynamic characterization, building a closer analogy to [54]:

$\begin{array}{lll}\text { interior corner } & \longleftrightarrow & \text { sink } \\ \text { hole } & \longleftrightarrow & \text { contact defect } \\ \text { step } & \longleftrightarrow & \text { transmission defect } \\ \text { exterior corner } & \longleftrightarrow \text { source }\end{array}$

Thinking in terms of interfacial energy, energy is lost at a sink, generated at a source, transmitted at a transmission defect and preserved at a contact defect.

There is yet another motivation for this terminology. We will later see that all defects possess a natural characterization as heteroclinic and homoclinic orbits. In this terminology, they coincide with the localized defects in spatially one-dimensional oscillatory media, which have been previously classified in the terminology of sink, contact, transmission, and source in [54]. To make the analogy clearer, transport of points on the interface has to be phrased in terms of group velocities. Since the dispersion relation $\lambda_{\mathrm{d}}(k)$ at the interface is symmetric in $k$, the group velocity $\lambda_{d}^{\prime}(0)$ in the tangential direction vanishes and transport is generated solely by geometry. In oscillatory media, transport is induced by group velocities of wave trains and described at small amplitudes by a viscous Burgers equation. Defects are then classified in [54] according to the relative slope of characteristics with respect to the speed of the defect. The correspondence actually goes much further, since spectra of linearized operators at corner defects and at defects in oscillatory media qualitatively agree.

Remark 2.9. Throughout the paper, we consider propagation in a direction normal to the primary interface. Most of the defects we find are actually symmetric with respect to $x \mapsto-x$. A slightly more general characterization of almost planar interfaces would allow for a propagation in the $x$-direction, as well. This would contribute a term $c_{x} \partial_{x} u$ in the equation, with an additional parameter $c_{x}$. Equivalently, we can rotate the plane by an angle $\varphi$ such that the speed of propagation points again in the $y$-direction.

We are now ready to state our main result of this section. 

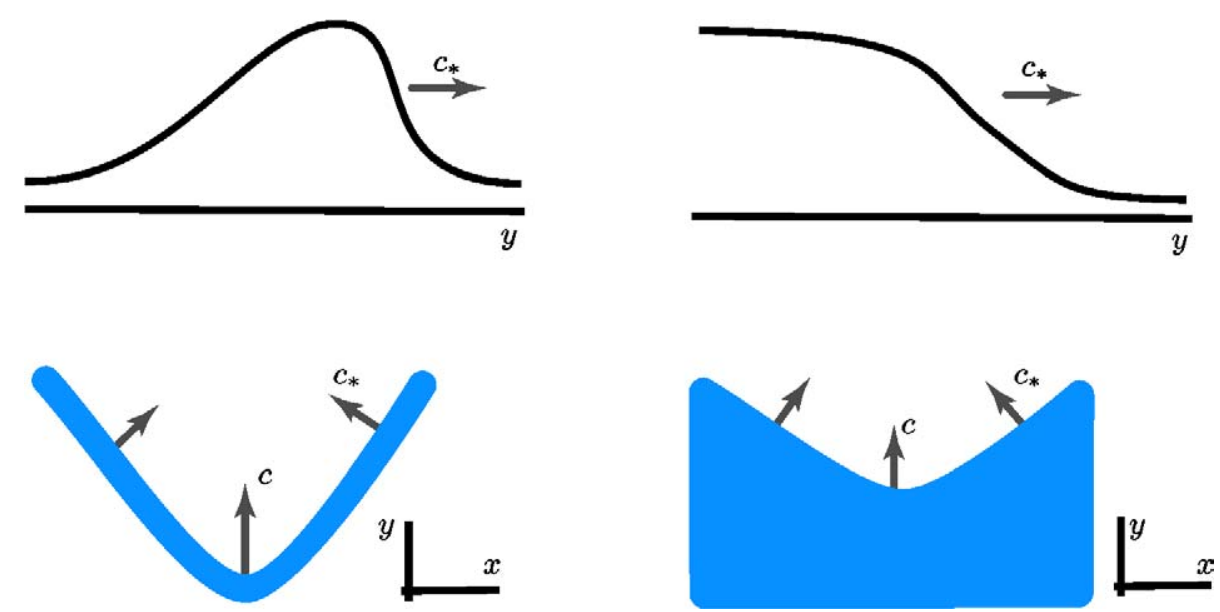

Fig. 3. One-dimensional pulses and fronts (top) generate two-dimensional corner defects in line- and in invasion patterns, respectively (bottom).

Theorem 1. Assume existence and zero-stability of a planar travelling wave $q_{*}(\cdot)$, Hypotheses 2.1 and 2.2. Then there is $\delta>0$ such that for each $c$ with $\left|c-c_{*}\right|<\delta, c>c_{*}$, there exists an interior corner defect. The defect is unique in the class of nontrivial almost planar corner defects up to translation in $x$. Moreover, it is invariant under reflection $x \mapsto x_{0}-x$ for an appropriate $x_{0}$ and is to leading order given through

$$
\begin{aligned}
& q(x, y ; c)=q_{*}(y+\xi(x))+\mathrm{O}\left(\left|c-c_{*}\right|\right), \\
& \xi^{\prime}(x)=\sqrt{\frac{2\left(c-c_{*}\right)}{c_{*}}} \tanh (\beta x)+\mathrm{O}\left(\left|c-c_{*}\right| \mathrm{e}^{-2|\beta x|}\right), \quad \beta=\frac{\sqrt{2 c_{*}\left(c-c_{*}\right)}}{\lambda_{\mathrm{d}}^{\prime \prime}(0)}<0 .
\end{aligned}
$$

For $c \leqslant c_{*}$, there are no nontrivial almost planar corner defects.

Note that the theorem does not require Hypothesis 2.3; see Remark 2.6(i).

We give a sketch of these interior corner defects in Fig. 3; see also Fig. 4. Note that the speed of propagation of the asymptotically planar interface with angle $\vartheta=\arctan \eta_{ \pm}$is given by the simple geometric condition $c=$ $c_{*} / \cos \vartheta$; see also [3] and the references therein. The existence and nonexistence part in Theorem 1 coincide with the results in [3] for scalar reaction-diffusion systems. We emphasize, however, that the results there cover large angles $\vartheta$, as well.

We outline first the proof of Theorem 1. We rewrite the travelling-wave equation (2.2) as a dynamical system in the direction $x$, perpendicular to the direction of propagation. We then parameterize solutions similarly to (2.11) exploiting the translation invariance $y \mapsto y+\xi$ of (2.2). The main step then is a dynamic center-manifold reduction to a two-dimensional center-manifold diffeomorphic to a strip $(\xi, \eta) \in \mathbb{R} \times(-\delta, \delta)$ and flow given by

$$
\xi^{\prime}=\eta+\mathrm{O}\left(\left|c-c_{*}\right||\eta|+|\eta|^{3}\right), \quad \eta^{\prime}=\frac{2}{\lambda_{\mathrm{d}}^{\prime \prime}(0)}\left(c-c_{*}-\frac{c_{*}}{2} \eta^{2}\right)+\mathrm{O}\left(\left|c-c_{*}\right|^{2}+|\eta|^{4}\right) .
$$

In particular, the right-hand sides of these equations do not depend on $\xi$. Moreover, they commute with the reversibility symmetry $x \mapsto-x, \xi \mapsto \xi, \eta \mapsto-\eta$. Bounded solutions are the well-known Burgers shocks.

Note that the reduced equation reflects steady-state profiles of the viscous Burgers' equation

$$
\eta_{t}=-\lambda_{\mathrm{d}}^{\prime \prime}(0) \eta_{x x}-c_{*}\left(\eta^{2}\right)_{x}
$$

This equation has been derived formally as a modulation equation for planar interfaces, previously, but we are not aware of any rigorous results in this direction; see however [11] for a justification in a different context. The 


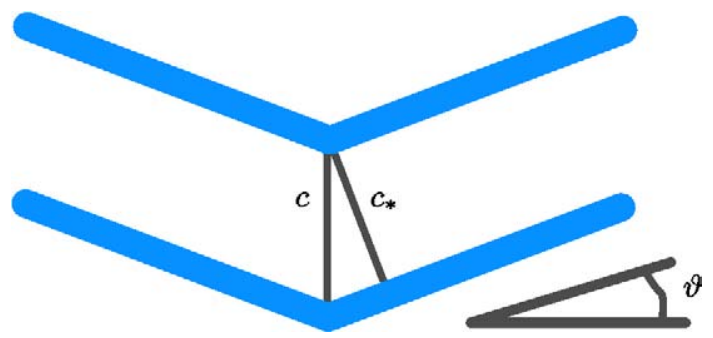

Fig. 4. Interface plotted at time $t=0$ and time $t=1$. The normal speed of propagation $c_{*}$ and the speed of the defect form a rectangular triangle with angle $\vartheta$.

Rankine-Hugoniot condition for the jump of the shock reduces to the purely geometric condition that the speed of the corner is determined by the condition that the orthogonal projection of the velocity of the corner on the (exterior) normal of the interfaces on both sides equals $c_{*}$; see Fig. 4.

Proof of Theorem 1. We rewrite the travelling-wave equation (2.2) as a first-order system in $x$

$$
\begin{aligned}
& u_{x}=v, \\
& v_{x}=-\partial_{y y} u-D^{-1}\left(c \partial_{y} u+f(u)\right),
\end{aligned}
$$

on the Hilbert space $Y=\left(H^{1} \times L^{2}\right)\left(\mathbb{R}, \mathbb{R}^{N}\right)$, or, in short notation,

$$
\boldsymbol{u}_{x}=\mathcal{A}(c) \boldsymbol{u}+\mathcal{F}(\boldsymbol{u}),
$$

where $\boldsymbol{u}=(u, v)^{T}$,

$$
\mathcal{A}(c)=\left(\begin{array}{cc}
0 & \mathrm{id} \\
-\partial_{y y}-D^{-1} c \partial_{y} & 0
\end{array}\right), \quad \mathcal{F}(\boldsymbol{u})=\left(\begin{array}{c}
0 \\
-D^{-1} f(u)
\end{array}\right) .
$$

Note that $\mathcal{A}(c)$ is closed on $Y$ with domain of definition $Y^{1}=\left(H^{2} \times H^{1}\right)\left(\mathbb{R}, \mathbb{R}^{N}\right)$. The nonlinearity $\mathcal{F}$ is smooth as a map from $Y$ to $Y$.

Eq. (2.16) possesses a continuous translation symmetry, induced by the $y$-shift $\xi: \boldsymbol{u}(\cdot) \mapsto \boldsymbol{u}(\cdot+\xi)$. Moreover, the equation possesses a reversibility symmetry $(u, v)^{T} \mapsto R(u, v)^{T}:=(u,-v)^{T}, x \mapsto-x$.

Hypothesis 2.1 on existence of planar interfaces shows that (2.17) possesses a family of equilibria

$$
\boldsymbol{q}_{*}^{\xi}=\left(\begin{array}{c}
q_{*}^{\xi}(\cdot) \\
0
\end{array}\right)=\left(\begin{array}{c}
q_{*}(\cdot+\xi) \\
0
\end{array}\right)
$$

The linearization of (2.16) about $\boldsymbol{q}_{*}^{0}$ is given by the operator

$$
\mathcal{A}_{*}=\left(\begin{array}{cc}
0 & \mathrm{id} \\
\mathcal{L}_{*} & 0
\end{array}\right)
$$

with $\mathcal{L}_{*}$ the linear operator defined in (2.6). Note that $\mathcal{A}_{*}$ is also closed in $Y$ with domain of definition $Y^{1}$. Due to the second order structure of (2.16), the spectrum of $\mathcal{A}_{*}$ coincides with the multi-valued squareroot of the spectrum of $\mathcal{L}_{*}$. From Hypothesis 2.2 we therefore conclude that

$$
\operatorname{spec} \mathcal{A}_{*} \cap\{|\operatorname{Re} \lambda| \leqslant \epsilon\}=\{0\},
$$

for some $\epsilon>0$, and that

$$
\left|\left(\mathrm{i} k-\mathcal{A}_{*}\right)^{-1}\right|_{Y \rightarrow Y} \leqslant \frac{C(1+|k|)}{|k|^{2}}, \quad \text { for any } k \in \mathbb{R}^{*},
$$


for some $C>0$. The eigenvalue in the origin is algebraically double with kernel and generalized kernel spanned by

$$
\operatorname{Ker} \mathcal{A}_{*}=\operatorname{span}\left(\boldsymbol{e}_{0}\right), \quad \boldsymbol{e}_{0}=\left(\begin{array}{c}
q_{*}^{\prime}(\cdot) \\
0
\end{array}\right), \quad \operatorname{gker} \mathcal{A}_{*}=\operatorname{span}\left(\boldsymbol{e}_{0}, \boldsymbol{e}_{1}\right), \quad \boldsymbol{e}_{1}=\left(\begin{array}{c}
0 \\
q_{*}^{\prime}(\cdot)
\end{array}\right),
$$

with $\mathcal{A}_{*} \boldsymbol{e}_{1}=\boldsymbol{e}_{0}$.

In order to find the spectral projection onto the generalized kernel, we construct the $L^{2} \times L^{2}$-adjoint

$$
\mathcal{A}_{*}^{\mathrm{ad}}=\left(\begin{array}{cc}
0 & \mathcal{L}_{*}^{\mathrm{ad}} \\
\mathrm{id} & 0
\end{array}\right),
$$

where $\mathcal{L}_{*}^{\text {ad }}$ is the $L^{2}$-adjoint of $\mathcal{L}_{*}$. The kernel and generalized kernel are given by

$$
\operatorname{Ker} \mathcal{A}_{*}^{\mathrm{ad}}=\operatorname{span}\left(\boldsymbol{e}_{0}^{\mathrm{ad}}\right), \quad \boldsymbol{e}_{0}^{\mathrm{ad}}=\left(\begin{array}{c}
0 \\
q_{*}^{\mathrm{ad}}(\cdot)
\end{array}\right), \quad \operatorname{gker} \mathcal{A}_{*}^{\mathrm{ad}}=\operatorname{span}\left(\boldsymbol{e}_{0}^{\mathrm{ad}}, \boldsymbol{e}_{1}^{\mathrm{ad}}\right), \quad \boldsymbol{e}_{1}^{\mathrm{ad}}=\left(\begin{array}{c}
q_{*}^{\mathrm{ad}}(\cdot) \\
0
\end{array}\right),
$$

with $\mathcal{A}_{*}^{\text {ad }} \boldsymbol{e}_{1}^{\text {ad }}=\boldsymbol{e}_{0}^{\text {ad }}$ and $\mathcal{L}_{*}^{\text {ad }} q_{*}^{\text {ad }}=0$. We assume that the adjoint eigenvectors are normalized such that

$$
\left\langle\boldsymbol{e}_{j}^{\mathrm{ad}}, \boldsymbol{e}_{j}\right\rangle_{L^{2} \times L^{2}}=0, \quad\left\langle\boldsymbol{e}_{j}^{\mathrm{ad}}, \boldsymbol{e}_{1-j}\right\rangle_{L^{2} \times L^{2}}=1, \quad j=0,1,
$$

by taking $\left(q_{*}^{\text {ad }}, q_{*}^{\prime}\right)_{L^{2}}=1$. The projection on the generalized kernel is then given as a bounded operator on $Y$ through

$$
P: Y \rightarrow Y, \quad P \boldsymbol{u}=\left\langle\boldsymbol{e}_{1}^{\mathrm{ad}}, \boldsymbol{u}\right\rangle_{L^{2} \times L^{2}} \boldsymbol{e}_{0}+\left\langle\boldsymbol{e}_{0}^{\mathrm{ad}}, \boldsymbol{u}\right\rangle_{L^{2} \times L^{2}} \boldsymbol{e}_{1} .
$$

Similarly, to the shifted equilibria $\boldsymbol{q}_{*}^{\xi}$ we introduce the shifted linear operator $\mathcal{A}_{*}^{\xi}$, the shifted eigenvector $\boldsymbol{e}_{0}^{\xi}=$ $\left(\left(q_{*}^{\prime}\right)^{\xi}(\cdot), 0\right)^{T}$, and analogously $\boldsymbol{e}_{1}^{\xi}, \boldsymbol{e}_{j}^{\mathrm{ad}, \xi}, P^{\xi}, \mathcal{A}_{*}^{\mathrm{ad}, \xi}$.

Following the general strategy for reduction around a continuous family of equilibria [55], we decompose

$$
\boldsymbol{u}=\boldsymbol{q}_{*}^{\xi}+\eta \boldsymbol{e}_{1}^{\xi}+\boldsymbol{w}^{\xi}, \quad \text { with } P^{\xi} \boldsymbol{w}^{\xi}=P \boldsymbol{w}=0 .
$$

Here $\xi$ and $\eta$ are real functions depending upon $x$. This provides us with coordinates in a neighborhood of the family of equilibria $\boldsymbol{q}_{*}^{\xi}$. Substituting (2.21) into (2.17), we find

$$
\xi_{x} \boldsymbol{e}_{0}^{\xi}+\eta_{x} \boldsymbol{e}_{1}^{\xi}+\eta \xi_{x} \partial_{\xi} \boldsymbol{e}_{1}^{\xi}+\left(\boldsymbol{w}^{\xi}\right)_{x}=\mathcal{A}_{*}^{\xi}\left(\eta \boldsymbol{e}_{1}^{\xi}+\boldsymbol{w}^{\xi}\right)+\left(\mathcal{A}(c)-\mathcal{A}\left(c_{*}\right)\right)\left(\boldsymbol{q}_{*}^{\xi}+\boldsymbol{w}^{\xi}\right)+\mathcal{G}^{\xi}\left(\boldsymbol{w}^{\xi}\right),
$$

where

$$
\mathcal{G}^{\xi}\left(\boldsymbol{w}^{\xi}\right)=\mathcal{F}\left(\boldsymbol{q}_{*}^{\xi}+\boldsymbol{w}^{\xi}\right)-\mathcal{F}\left(\boldsymbol{q}_{*}^{\xi}\right)-D_{\boldsymbol{u}} \mathcal{F}\left(\boldsymbol{q}_{*}^{\xi}\right) \boldsymbol{w}^{\xi},
$$

such that $\mathcal{G}^{\xi}$ is smooth on $Y$ and $\mathcal{G}^{\xi}(\boldsymbol{w})=\mathrm{O}\left(|\boldsymbol{w}|_{Y}^{2}\right)$, uniformly in $\xi$. Notice that $\mathcal{G}^{\xi}$ does not depend upon $\eta$ since the first component of $\boldsymbol{e}_{1}$ is zero.

We next take the scalar product of (2.22) with $\boldsymbol{e}_{1}^{\mathrm{ad}, \xi}$ and exploit the fact that the second component of $\boldsymbol{e}_{1}^{\mathrm{ad}, \xi}$ vanishes to obtain

$$
\xi_{x}+\left\langle\boldsymbol{e}_{1}^{\mathrm{ad}, \xi},\left(\boldsymbol{w}^{\xi}\right)_{x}\right\rangle=\eta
$$

From $\left\langle\boldsymbol{e}_{1}^{\mathrm{ad}, \xi}, \boldsymbol{w}^{\xi}\right\rangle_{x}=0$ and the invariance of the $L^{2} \times L^{2}$-scalar product under the $y$-shift $(\cdot)^{\xi}$ we obtain

$$
\left\langle\boldsymbol{e}_{1}^{\mathrm{ad}, \xi},\left(\boldsymbol{w}^{\xi}\right)_{x}\right\rangle=-\left\langle\xi_{x} \partial_{\xi} \boldsymbol{e}_{1}^{\mathrm{ad}, \xi}, \boldsymbol{w}^{\xi}\right\rangle=-\left\langle\xi_{x} \partial_{\xi} \boldsymbol{e}_{1}^{\mathrm{ad}}, \boldsymbol{w}\right\rangle
$$

We therefore find

$$
\xi_{x}=\left(1-\left\langle\partial_{\xi} \boldsymbol{e}_{1}^{\mathrm{ad}}, \boldsymbol{w}\right\rangle\right)^{-1} \eta=\eta+\mathrm{O}\left(|\eta||\boldsymbol{w}|_{Y}\right) .
$$


Taking the scalar product of (2.22) with $\boldsymbol{e}_{0}^{\text {ad, } \xi}$ yields

$$
\eta_{x}+\eta \xi_{x}\left\langle\boldsymbol{e}_{0}^{\mathrm{ad}}, \partial_{\xi} \boldsymbol{e}_{1}\right\rangle+\left\langle\boldsymbol{e}_{0}^{\mathrm{ad}, \xi},\left(\boldsymbol{w}^{\xi}\right)_{x}\right\rangle=\left\langle\boldsymbol{e}_{0}^{\mathrm{ad}},\left(\mathcal{A}(c)-\mathcal{A}\left(c_{*}\right)\right)\left(\boldsymbol{q}_{*}^{0}+\boldsymbol{w}\right)\right\rangle+\left\langle\boldsymbol{e}_{0}^{\mathrm{ad}}, \mathcal{G}^{0}(\boldsymbol{w})\right\rangle,
$$

in which we used the invariance of the $L^{2} \times L^{2}$-scalar product under the $y$-shift $(\cdot)^{\xi}$. We claim that

$$
\left\langle\boldsymbol{e}_{0}^{\mathrm{ad}}, \partial_{\xi} \boldsymbol{e}_{1}\right\rangle=\frac{c_{*}}{\lambda_{\mathrm{d}}^{\prime \prime}(0)} .
$$

To see this, first note that

$$
\left\langle\boldsymbol{e}_{0}^{\mathrm{ad}}, \partial_{\xi} \boldsymbol{e}_{1}\right\rangle=\left\langle q_{*}^{\mathrm{ad}}, q_{*}^{\prime \prime}\right\rangle=\frac{1}{2}\left(\left\langle q_{*}^{\mathrm{ad}}, q_{*}^{\prime \prime}\right\rangle-\left\langle q_{*}^{\mathrm{ad}^{\prime}}, q_{*}^{\prime}\right\rangle\right) .
$$

A short, direct calculation shows that

$$
\frac{\mathrm{d}}{\mathrm{d} y}\left(q_{*}^{\mathrm{ad}} \cdot q_{*}^{\prime \prime}-q_{*}^{\mathrm{ad}^{\prime}} \cdot q_{*}^{\prime}\right)=-\frac{\mathrm{d}}{\mathrm{d} y}\left(D^{-1} c_{*} q_{*}^{\mathrm{ad}} \cdot q_{*}^{\prime}\right) .
$$

Integration with respect to $y$ gives

$$
\left\langle q_{*}^{\mathrm{ad}}, q_{*}^{\prime \prime}\right\rangle-\left\langle q_{*}^{\mathrm{ad}^{\prime}}, q_{*}^{\prime}\right\rangle=-c_{*}\left\langle D^{-1} q_{*}^{\mathrm{ad}}, q_{*}^{\prime}\right\rangle .
$$

Finally, if we take the scalar product of (2.9) with $D^{-1} q_{*}^{\text {ad }}$ and exploit the fact that the left hand side vanishes, we obtain

$$
\left\langle D^{-1} q_{*}^{\text {ad }}, q_{*}^{\prime}\right\rangle=-\frac{2}{\lambda_{\mathrm{d}}^{\prime \prime}(0)},
$$

which proves our claim (2.25).

As already observed before, we have $\left\langle\boldsymbol{e}_{0}^{\mathrm{ad}, \xi},\left(\boldsymbol{w}^{\xi}\right)_{x}\right\rangle=-\xi_{x}\left\langle\partial_{\xi} \boldsymbol{e}_{0}^{\mathrm{ad}}, \boldsymbol{w}\right\rangle$, and

$$
\left\langle\boldsymbol{e}_{0}^{\mathrm{ad}},\left(\mathcal{A}(c)-\mathcal{A}\left(c_{*}\right)\right) \boldsymbol{q}_{*}^{0}\right\rangle=-\left(c-c_{*}\right)\left\langle q_{*}^{\mathrm{ad}}, D^{-1} q_{*}^{\prime}\right\rangle=\frac{2}{\lambda_{\mathrm{d}}^{\prime \prime}(0)}\left(c-c_{*}\right),
$$

such the equality (2.24) can be rewritten as

$$
\eta_{x}=\frac{2}{\lambda_{\mathrm{d}}^{\prime \prime}(0)}\left(c-c_{*}\right)-\frac{c_{*}}{\lambda_{\mathrm{d}}^{\prime \prime}(0)} \xi_{x} \eta+\xi_{x}\left\langle\partial_{\xi} \boldsymbol{e}_{0}^{\mathrm{ad}}, \boldsymbol{w}\right\rangle+\left\langle\boldsymbol{e}_{0}^{\mathrm{ad}},\left(\mathcal{A}(c)-\mathcal{A}\left(c_{*}\right)\right) \boldsymbol{w}+\mathcal{G}^{0}(\boldsymbol{w})\right\rangle .
$$

Note that this becomes a first order differential equation for $\eta$ once we substitute the expression for $\xi_{x}$ from (2.23).

Having derived equations for $\xi_{x}$ and $\eta_{x}$, we now derive an equation for $\boldsymbol{w}_{x}$, the hyperbolic part. We therefore project (2.22) with (id $\left.-P^{\xi}\right)$ onto the hyperbolic subspace of $\mathcal{A}_{*}$ and find

$$
\boldsymbol{w}_{x}=-\xi_{x} \partial_{\xi} \boldsymbol{w}-\xi_{x}\left(\partial_{\xi} P\right) \boldsymbol{w}+\mathcal{A}_{*} \boldsymbol{w}+(\mathrm{id}-P)\left(-\eta \xi_{x} \partial_{\xi} \boldsymbol{e}_{1}+\left(\mathcal{A}(c)-\mathcal{A}\left(c_{*}\right)\right)\left(\boldsymbol{q}_{*}^{0}+\boldsymbol{w}\right)+\mathcal{G}^{0}(\boldsymbol{w})\right) .
$$

If we substitute the expressions (2.27) for $\eta_{x}$ and (2.23) for $\xi_{x}$, this equality provides us with a first order differential equation for $\boldsymbol{w}$.

Summarizing, we have found a first order quasilinear system of differential equations

$$
\begin{aligned}
& \eta_{x}=\frac{2}{\lambda_{\mathrm{d}}^{\prime \prime}(0)}\left(c-c_{*}\right)-\frac{c_{*}}{\lambda_{\mathrm{d}}^{\prime \prime}(0)} \eta^{2}+\mathrm{O}\left(\left|c-c_{*}\right||\boldsymbol{w}|_{Y}+|\eta||\boldsymbol{w}|_{Y}+|\boldsymbol{w}|_{Y}^{2}\right), \\
& \boldsymbol{w}_{x}=\mathcal{A}_{*} \boldsymbol{w}+\mathrm{O}\left(\left|c-c_{*}\right|+|\eta|^{2}+|\boldsymbol{w}|_{Y}^{2}+|\eta||\boldsymbol{w}|_{Y^{1}}\right),
\end{aligned}
$$

posed on the Hilbert space $\mathbb{R} \times Y_{h}$, with $Y_{h}=(\mathrm{id}-P) Y$. A key point in this choice of coordinates is that this system is independent of $\xi$, such that we can solve for $\xi$, separately. Since $P \boldsymbol{w}=0$, the linearized equation is hyperbolic in the $\boldsymbol{w}$-component. We may now evoke a center-manifold reduction theorem for quasilinear systems [45, Theorem 1] to conclude that for $c$ close to $c_{*}$, all solutions with $\eta, \boldsymbol{w}$ sufficiently small are solutions to a 
reduced differential equation for $\eta$, obtained by substituting $\boldsymbol{w}=h\left(\boldsymbol{\eta} ; c-c_{*}\right)=\mathrm{O}\left(\left|c-c_{*}\right|+|\eta|^{2}\right)$ into (2.28). The reduction theorem ensures that all nonlinear functions and their dependences on parameters are of class $C^{k}$ for an arbitrary but fixed $k<\infty$. Moreover, the reduction procedure preserves the action of the reversibility $R$, such that the function $h$ commutes with the action of $R$ restricted to center and hyperbolic subspaces. Since $R \boldsymbol{e}_{1}=-\boldsymbol{e}_{1}$, we can conclude that the right-hand side of the reduced equation on the center subspace is an even function of $\eta$, with expansion

$$
\eta_{x}=\frac{2}{\lambda_{\mathrm{d}}^{\prime \prime}(0)}\left(c-c_{*}-\frac{c_{*}}{2} \eta^{2}\right)+\mathrm{O}\left(\left|c-c_{*}\right|^{2}+|\eta|^{4}\right) .
$$

We can determine the location of the equilibria in (2.30) a priori and to any order if we exploit the rotational invariance of the reaction-diffusion system in the steady frame (2.3). Together with the primary plane interface, there are rotated planar interface, parameterized by the angle $\vartheta$ relative to the original planar interface. In our reduced system (2.30), these rotated interfaces yield equilibria $\eta=\tan \vartheta$ (here $\eta>0$ corresponds to the rotated interface being shifted backwards as $x$ increases). The rotated planar interfaces travel with normal speed $c_{\perp}=c_{*}$. The (nonnormal) speed of propagation $c$ in the $y$-direction, imposed in (2.2), induces a projected normal speed of propagation $c_{\perp}=c \cos \vartheta$; see Fig. 4. Therefore the equilibria to (2.30) are explicitly given by

$$
\eta_{ \pm}^{2}=\frac{c^{2}-c_{*}^{2}}{c^{2}}
$$

Nontrivial, small bounded solutions for the scalar reduced ODE (2.30) exist precisely when there are at least two equilibria, that is, when $c>c_{*}$. All small solutions are then given by either one of the equilibria, or the heteroclinic orbit connecting $\eta_{+}<0$, as $x \rightarrow \infty$, with $\eta_{-}>0$, as $x \rightarrow-\infty$. It is straightforward to verify that, to leading order, the heteroclinic takes the explicit form (2.13). This concludes the proof of Theorem 1.

We conclude this section with several examples of reaction-diffusion systems for which such corner defects exist.

The simplest example is found in the classical Nagumo equation

$$
u_{t}=\Delta u+u(u-a)(1-u) .
$$

Existence and stability of propagating fronts, Hypotheses 2.1 and 2.2 are satisfied for $a \in(0,1), a \neq 1 / 2$, since the front is stable in one space-dimension and the diffusion matrix is trivial.

Another example is provided by the Gray-Scott equation

$$
\begin{aligned}
& \partial_{t} u_{1}=d_{1} \Delta u_{1}-u_{1} u_{2}^{2}+F\left(1-u_{1}\right), \\
& \partial_{t} u_{2}=d_{2} \Delta u_{2}+u_{1} u_{2}^{2}-(F+k) u_{1} .
\end{aligned}
$$

For equal diffusion constants and particular parameter values $k, F$, an explicit expression for a front solution was given in $[18,19]$. Numerical evidence strongly suggests that the planar fronts are asymptotically stable with dispersion relation $\lambda_{\mathrm{d}}^{\prime \prime}(0)<0$, although no analytical proof of this fact seems to be available.

Existence and stability of a one-dimensional pulse is known for the FitzHugh-Nagumo equation [1,26,32,60,67]

$$
\begin{aligned}
& \partial_{t} u_{1}=\Delta u_{1}+u_{1}\left(1-u_{1}\right)\left(u_{1}-a\right)-u_{2}, \\
& \partial_{t} u_{2}=\delta \Delta u_{2}+\varepsilon\left(u_{1}-\gamma u_{2}\right),
\end{aligned}
$$

where $\delta \geqslant 0$ and $\varepsilon$ are sufficiently small, $0<a<1 / 2$, and $\gamma$ is large.

Lemma 2.10 ([60]). The (fast) planar pulse for the FitzHugh-Nagumo equation with $0 \leqslant \delta \ll \varepsilon \ll 1$ is stable in two space-dimensions, that is $\lambda_{\mathrm{d}}^{\prime \prime}(0)<0$. In particular, Hypotheses 2.1 and 2.2 hold for $\delta>0$, sufficiently small. 
Proof. The proof for $\delta=0$ is a consequence of the discussion in [60], where arbitrary periodic boundary conditions in the $x$-direction are imposed. A perturbation argument similar to the one presented in [1] shows that the same stability properties hold for $\delta>0$, as well.

As an immediate consequence, Theorem 1 shows that interior corner defects actually exist as pure travellingwave solutions. They are observed typically at collision points of wave-trains emitted by spiral waves; see [66] for illustration and background on spiral waves in the Belousov-Zhabotinsky reaction.

A minor modification actually allows us to apply Theorem 1 to the case $\delta=0$, as well. Therefore, note that in the travelling-wave equation

$$
\begin{aligned}
& c \partial_{y} u_{1}=\Delta u_{1}+u_{1}\left(1-u_{1}\right)\left(u_{1}-a\right)-u_{2}, \\
& c \partial_{y} u_{2}=\varepsilon\left(u_{1}-\gamma u_{2}\right),
\end{aligned}
$$

we may solve the second equation for $u_{1}$ as a (nonlocal) function of $u_{2}$ and end up with a single travelling-wave equation. The proof of Theorem 1 then goes through for this single equation.

A number of fronts satisfy Hypothesis 2.2 only in function spaces equipped with an exponential weight. In oversimplified models for combustion, $u$ is scalar and $f(u) \equiv 0$ for $u \in[0, \delta], f(1)=0$, and $f(u)>0$ on $(\delta, 1)$ [3]. The reaction front connecting $u=1$ at $-\infty$ to $u=0$ then satisfies Hypothesis 2.2 on stability in a space $X_{\eta} \subset L_{\text {loc }}^{2}$ of functions with exponential weight

$$
|u|_{X_{\eta}}^{2}:=\int_{\mathbb{R}}\left|u(y)\left(1+\mathrm{e}^{\eta y}\right)\right|^{2} \mathrm{~d} y,
$$

for some positive rate $\eta>0$.

A simple example is provided by a variant of the Nagumo equation (2.31) when $a<0$. For $c_{*}=\sqrt{2}\left(\frac{1}{2}-a\right)$, there exists an explicit front solution, which satisfies Hypothesis 2.2 in $X_{\eta}$ for $\eta \in(1 / \sqrt{2},-2 a / \sqrt{2})$ if $1<-2 a$ and $\eta \in(-2 a / \sqrt{2}, 1 / \sqrt{2})$ if $1>-2 a$; see [62]. Actually, fronts exist for all $c \geqslant 2 \sqrt{-a}$. However, all fronts with $c<c_{*}$ are unstable in any exponentially weighted function space, whereas the front with $c=c_{*}$ is the unique front which possesses a single eigenvalue $\lambda=0$ in an exponentially weighted spaces. More information on the selection mechanisms for fronts can be found in [62]. In the terminology employed there, we construct interior corners for pushed fronts. We do not know if interior corners exist for pulled fronts; see also [63, §5.2]

Another example is provided by the cubic-quintic Ginzburg-Landau equation for complex valued function $A$ and parameters $\mu, \gamma \in \mathbb{R}$,

$$
A_{t}=\Delta A+\mu A+\gamma A|A|^{2}-A|A|^{4} .
$$

Of interest are fronts between patterned states $A(y)=|A| \mathrm{e}^{\mathrm{i}\left(k_{x} x+k_{y} y\right)}$ and the trivial solution in this equation. Here, we specialize to real solutions satisfying

$$
u_{t}=\Delta u+\mu u+\gamma u^{3}-u^{5},
$$

and briefly discuss the case $k \neq 0$ in Section 6. For $\mu<0$ and $\gamma>0$, there exist fronts of the same type as in the Nagumo equation connecting the (stable) trivial solution $u=0$ to the zero $u_{+}$of $\mu u+\gamma u^{3}-u^{5}$ with maximal modulus. If $\mu>0$, the origin is unstable, but there may still be interfaces between $u_{+}$spreading into $u=0$. Phaseplane analysis and Sturm-Liouville theory show that for $\gamma>2 / \sqrt{3}$, a front solution of the type described for the Nagumo equation exists and satisfies Hypothesis 2.2.

Remark 2.11 (Periodic wave-trains). Theorem 1 can also be applied to $y$-periodic, $x$-independent wave-trains. For this, we impose periodic boundary conditions in the $y$-direction and follow the proof. Examples of wave-trains for which the stability hypothesis can be verified arise in the FitzHugh-Nagumo equation [13,53]. The transverse stability assumption in the regime of large period in $y$ can be concluded from the transverse stability of the pulse 
using the results in [53]. The spatial patterns that are described by the heteroclinics on the center manifold are periodic juxtapositions in the direction of propagation of the picture for a single interior corner, Fig. 2(a). We note that these types of patterns can be observed in spiral wave interaction, where wave trains, emitted from two spiral cores, collide.

\section{Stability of corners}

The goal of this section is to show stability of interior corner defects as found in Theorem 1 . We assume that Hypotheses 2.1 and 2.3 hold. Here, the strengthened stability Hypothesis 2.3 is needed as compared to the weaker zero stability Hypothesis 2.2. The results below show that interior corner defects are asymptotically stable under perturbations which are exponentially localized in the direction of the primary interface. Roughly speaking, localization refers to the position of the interface in Theorem 2 and to the angle in the change of the orientation of the interface in Theorem 3. The perturbations are fully localized in Theorem 2, and localized in each spatial direction except for those parallel to the asymptotic straight lines of the interface in Theorem 3.

In order to state the results we first introduce the space of exponentially localized functions $\mathcal{X}_{\eta} \subset L_{\text {loc }}^{2}\left(\mathbb{R}^{2}, \mathbb{R}^{N}\right.$ ) and $\mathcal{X}_{\eta}^{2} \subset H_{\text {loc }}^{2}\left(\mathbb{R}^{2}, \mathbb{R}^{N}\right)$, with norms

$$
\begin{aligned}
& |u|_{\mathcal{X}_{\eta}}^{2}:=\int_{x, y}|u(x, y) \cosh (\eta x)|^{2} \mathrm{~d} y \mathrm{~d} x<\infty, \\
& |u|_{\mathcal{X}_{\eta}^{2}}^{2}:=|u|_{\mathcal{X}_{\eta}}^{2}+|\Delta u|_{\mathcal{X}_{\eta}}^{2} .
\end{aligned}
$$

Note that perturbations contained in $\mathcal{X}_{\eta}$ change the local shape of the corner but do not alter the position of the asymptotic straight lines of the interface

Theorem 2. Assume existence and stability of a planar travelling wave, Hypotheses 2.1 and 2.3. Then there exist positive constants $\varepsilon_{0}$ and $\eta_{0}$ such that the (unique) interior corner defects $q(\cdot, \cdot ; c)$ ), described in Theorem 1 for $c-c_{*} \in\left(0, \varepsilon_{0}\right)$ are asymptotically stable under perturbations $u_{0} \in \mathcal{X}_{\eta}^{2}$, as defined in (3.1)-(3.2), for all $0<\eta<$ $\eta_{0}\left(c-c_{*}\right)^{1 / 2}$. More precisely, there exist positive constants $\delta=\delta(\eta, c), d=d(\eta, c)$, and $C=C(\eta, c)$ such that for any initial condition $q(\cdot, \cdot ; c)+u_{0}$ with $\left|u_{0}\right|_{\mathcal{X}_{\eta}^{2}}<\delta$, the solution $u$ to (2.1) satisfies

$$
|u(t, \cdot, \cdot)-q(\cdot, \cdot ; c)|_{\mathcal{X}_{\eta}^{2}} \leqslant C \delta \mathrm{e}^{-d t}
$$

for any $t>0$.

The stability properties are best understood in the context of the stability properties of the viscous shock profile $q(x)=\tanh (x / 2)$ in Burgers equation [56],

$$
u_{t}=u_{x x}+u u_{x} \text {. }
$$

In our context, the velocity $u$ corresponds to the inclination $\eta$ of the interface (see (2.15)), such that the actual difference between two solutions $u$ and $q$ is measured by the integral $\Phi=\int_{x} u$, which corresponds to the position $\xi$ of the interface. The variable $\Phi$ solves a viscous eikonal equation

$$
\Phi_{t}=\Phi_{x x}+\frac{1}{2} \Phi_{x}^{2}
$$

If we linearize (3.5) about $\Phi=\int_{x} q$, we find

$$
\Psi_{t}=\Psi_{x x}+\tanh \left(\frac{x}{2}\right) \Psi_{x} .
$$


This linear equation generates a contraction semi-group on the function space $X_{\eta_{-}, \eta_{+}}$with $-1<\eta_{-}<0<\eta_{+}<1$. Since nonlinearities are differentiable on interpolation spaces defined relative to the exponentially weighted spaces $X_{\eta_{-}, \eta_{+}}$, standard results on nonlinear semigroup theory [27] give an asymptotic stability result in the spirit of Theorem 2. Note however that the function $\Phi$ itself is not bounded and nonlinearities are well-defined since they do only depend on the derivative $\Phi$. An alternative proof could therefore operate on Burgers equation (3.4), on the same space of exponentially weighted functions. Localization of the perturbation in the $\Phi$-variables corresponds to zero mass perturbations in Burgers equation $\int u=0$. If we allow for nonzero mass, the linearization about the shock in Burgers equation possesses a neutral eigenvalue. Stability with respect to these perturbations now holds if we shift the profile $q\left(\cdot+x_{0}\right)$ by an appropriate constant $x_{0}$ to compensate for the additional mass. The next natural question now arises whether a similar result holds for the stability of corners.

We therefore introduce the function space $\tilde{\mathcal{X}}_{\eta}=\mathcal{X}_{\eta} \oplus \mathcal{X}_{\text {step }} \subset L_{\text {loc }}^{2}\left(\mathbb{R}^{2}, \mathbb{R}^{N}\right)$ with

$$
\mathcal{X}_{\text {step }}=\left\{u_{\text {step }}(x, y)=u_{-}(y)(1-\theta(x))+u_{+}(y) \theta(x) ; u_{ \pm} \in L^{2}\left(\mathbb{R}, \mathbb{R}^{N}\right)\right\} .
$$

Here, $\theta(\cdot)$ denotes the Heavyside step function. We equip $\tilde{\mathcal{X}}_{\eta}$ with the Euclidean product norm

$$
\left|u+u_{\text {step }}\right|_{\tilde{\mathcal{X}}_{\eta}}^{2}:=|u|_{\mathcal{X}_{\eta}}^{2}+\left|u_{-}\right|_{L^{2}}^{2}+\left|u_{+}\right|_{L^{2}}^{2} .
$$

We also consider $\tilde{\mathcal{X}}_{\eta}^{2}=\mathcal{X}_{\eta}^{2} \oplus \mathcal{X}_{\text {step }}^{2} \subset H_{\text {loc }}^{2}\left(\mathbb{R}^{2}, \mathbb{R}^{N}\right)$ with

$$
\mathcal{X}_{\text {step }}^{2}=(\text { id }-\Delta)^{-1} \mathcal{X}_{\text {step }} \subset H_{\text {loc }}^{2}\left(\mathbb{R}^{2}, \mathbb{R}^{N}\right) .
$$

Note that any function $v_{\text {step }} \in \mathcal{X}_{\text {step }}^{2}$ is obtained through

$$
v_{\text {step }}(x, y)=(\mathrm{id}-\Delta)^{-1}\left(u_{-}(y)(1-\theta(x))+u_{+}(y) \theta(x)\right), \quad u_{ \pm} \in L^{2}\left(\mathbb{R}, \mathbb{R}^{N}\right),
$$

and can be computed explicitly in terms of $u_{-}$and $u_{+}$with the help of the Green's function for (id $\left.-\Delta\right)^{-1}$. In particular, any $v_{\text {step }} \in \mathcal{X}_{\text {step }}^{2}$ belongs to $C^{1}\left(\mathbb{R}^{2}, \mathbb{R}^{N}\right)$ and has the asymptotic behavior

$$
v_{\text {step }}(x, y)=\left(\mathrm{id}-\partial_{y y}\right)^{-1} u_{-}(y)+\mathrm{O}\left(\mathrm{e}^{x}\left(\left|u_{-}\right|_{L^{2}}+\left|u_{+}\right|_{L^{2}}\right)\right),
$$

as $x \rightarrow-\infty$, and

$$
v_{\text {step }}(x, y)=\left(\mathrm{id}-\partial_{y y}\right)^{-1} u_{+}(y)+\mathrm{O}\left(\mathrm{e}^{-x}\left(\left|u_{-}\right|_{L^{2}}+\left|u_{+}\right|_{L^{2}}\right)\right),
$$

as $x \rightarrow \infty$. We equip $\tilde{\mathcal{X}}_{\eta}^{2}$ with the product norm

$$
\left|v+v_{\text {step }}\right|_{\tilde{\mathcal{X}}_{\eta}^{2}}^{2}:=|v|_{\mathcal{X}_{\eta}^{2}}^{2}+\left|v_{-}\right|_{H^{2}}^{2}+\left|v_{+}\right|_{H^{2}}^{2}
$$

where

$$
v_{ \pm}(\cdot)=\lim _{x \rightarrow \pm \infty} v_{\text {step }}(x, \cdot) \in H^{2}\left(\mathbb{R}, \mathbb{R}^{N}\right) .
$$

For $\eta \in(0,1)$ the operator $\Delta-$ id is sectorial on $\tilde{\mathcal{X}}_{\eta}$ with domain of definition $\tilde{\mathcal{X}}_{\eta}^{2}$ and its spectrum is upper semi-continuous with respect to the parameter $\eta$.

The interior corner $q(\cdot, \cdot ; c)$ found in Theorem 1 is of the form

$$
q(x, y ; c)=q_{*}(y+\xi(x))+\mathrm{O}\left(\left|c-c_{*}\right|\right)=: \tilde{q}(x, y+\xi(x) ; c),
$$

where $\xi(x)$ is the $y$-dependent position of the interface. Then $\tilde{q} \in \tilde{\mathcal{X}}_{\eta}^{2}$, and the same is true for the family of translates $q\left(x+x_{0}, y+y_{0} ; c\right)$, which generates a two-dimensional surface of equilibria to (2.1) in the function space $\tilde{\mathcal{X}}_{\eta}^{2}$ (this is not the case for the function space $\mathcal{X}_{\eta}^{2}$, since the differences $q\left(x+x_{0}, y ; c\right)-q(x, y ; c)$ and $q\left(x, y+y_{0} ; c\right)-q(x, y ; c)$ are not contained in $\left.\mathcal{X}_{\eta}^{2}\right)$. In Theorem 3 we consider perturbations of the form $u_{0}(x, y)=$ $\tilde{u}_{0}(x, y+\xi(x))$ with $\tilde{u}_{0}(\cdot, \cdot) \in \tilde{\mathcal{X}}_{\eta}^{2}$, and $\xi(x)$ given by the fixed reference interface. Then $u_{0}$ is asymptotically 
constant along the curves $y+\xi(x) \equiv$ const parallel to the interface, and localized elsewhere. Such perturbations allow for changing the position of the corner, but not its angle. Note that if $\tilde{u} \in \mathcal{X}_{\eta}$ then $u$ belongs to $\mathcal{X}_{\eta}$ as well, but this is not true for functions $u$ with $\tilde{u}$ in the extended space $\tilde{\mathcal{X}}_{\eta}$.

Remark 3.1. Initial conditions $u_{0}(x, y)=\tilde{u}_{0}(x, y+\xi(x))$ with $\tilde{u}_{0} \in \tilde{\mathcal{X}}_{\eta}^{2}$ as before could be also described using the $\xi$-dependent space $\mathcal{X}_{\eta} \oplus \tilde{\mathcal{X}}_{\text {step }}$,

$$
\tilde{\mathcal{X}}_{\text {step }}=\left\{\tilde{u}_{\text {step }}(x, y)=u_{-}\left(y+\eta_{*} x\right)(1-\theta(x))+u_{+}\left(y-\eta_{*} x\right) \theta(x) ; u_{ \pm} \in L^{2}\left(\mathbb{R}, \mathbb{R}^{N}\right)\right\},
$$

in which $\eta_{*}=\lim _{|x| \rightarrow \infty}\left|\xi^{\prime}(x)\right|$.

Theorem 3. Assume existence and stability of a planar travelling wave, Hypotheses 2.1 and 2.3. Then there exist positive constants $\varepsilon_{0}$ and $\eta_{0}$ such that the family of (unique) interior corner defects $q\left(\cdot+x_{0}, \cdot+y_{0} ; c\right.$ ), described in Theorem 1 is asymptotically stable with asymptotic phase, under perturbations $u_{0}$ with $\tilde{u}_{0} \in \tilde{\mathcal{X}}_{\eta}^{2}$, for all $0<\eta<$ $\eta_{0}\left(c-c_{*}\right)^{1 / 2}$ when $c-c_{*} \in\left(0, \varepsilon_{0}\right)$. More precisely, there exist positive constants $\delta=\delta(\eta, c), d=d(\eta, c)$, and $C=C(\eta, c)$ such that for any initial condition $q(x, y ; c)+\tilde{u}_{0}(x, y+\xi(x))$ with $\left|\tilde{u}_{0}\right|_{\tilde{\mathcal{X}}_{\eta}^{2}}<\delta$, there exist $x_{0}$ and $y_{0}$ such that the solution $u$ to (2.1) satisfies

$$
\left|u(t, \cdot, \cdot)-q\left(\cdot+x_{0}, \cdot+y_{0} ; c\right)\right|_{\tilde{\mathcal{X}}_{\eta}^{2}} \leqslant C \delta \mathrm{e}^{-d t},
$$

for all $t>0$.

The remainder of this section is occupied by the proofs for Theorems 2 and 3 . The main part of the analysis is concerned with the study of the spectrum of the linearization about the interior corner,

$$
\mathcal{L} u=D \Delta u+c \partial_{y} u+f^{\prime}(q(\cdot, \cdot ; c)) u,
$$

where the function $q(\cdot, \cdot ; c)$ is given by Theorem 1 on existence of interior corners for wave speeds $c>c_{*}$. In the case of fully localized perturbations, Theorem 2, we consider $\mathcal{L}$ on $\mathcal{X}_{\eta}$ with domain of definition $\mathcal{X}_{\eta}^{2}$ (Proposition 3.2). For Theorem 3 we have to consider a slightly modified operator. Recall that in this case the perturbations are of the form

$$
u(x, y)=\tilde{u}(x, y+\xi(x))=: \tilde{u}^{\xi}(x, y),
$$

with $\tilde{u} \in \tilde{\mathcal{X}}_{\eta}^{2}$. On the linear level, the stability analysis reduces to the eigenvalue problem

$$
(\mathcal{L}-\lambda \text { id }) \tilde{u}^{\xi}=\tilde{h}^{\xi}
$$

for $\tilde{u} \in \tilde{\mathcal{X}}_{\eta}^{2}$ and $\tilde{h} \in \tilde{\mathcal{X}}_{\eta}$. We may then transform the independent variables to obtain

$$
(\tilde{\mathcal{L}}-\lambda \mathrm{id}) \tilde{u}=\tilde{h},
$$

where

$$
\tilde{\mathcal{L}} \tilde{u}=D\left(\left(\partial_{x}+\xi_{x} \partial_{y}\right)^{2}+\partial_{y y}\right) \tilde{u}+c \partial_{y} \tilde{u}+f^{\prime}(\tilde{q}(\cdot, \cdot ; c)) \tilde{u},
$$

and $\tilde{q}(x, y+\xi(x) ; c)=q(x, y ; c)$ (on $\mathcal{X}_{\eta}$ the two eigenvalues problems are equivalent). Note that $\tilde{\mathcal{L}}$ is a small, relatively bounded perturbation of the linearization $\mathcal{M}_{*}$ about $q_{*}$ given in (2.7); see (3.7). Again, we decompose the proof into a statement on the linearized equation (Proposition 3.4) and a short separate argument for the nonlinear part.

Proposition 3.2. Under the assumptions of Theorem 2, there exist positive constants $\varepsilon_{0}, \eta_{0}$ and $\delta_{0}$ such that for any $c-c_{*} \in\left(0, \varepsilon_{0}\right)$, and any weight $\eta=\tilde{\eta}\left(c-c_{*}\right)^{1 / 2}$ with $\tilde{\eta} \in\left(0, \eta_{0}\right)$, the spectrum of $\mathcal{L}$ in $\mathcal{X}_{\eta}$ satisfies

$\operatorname{spec} \mathcal{L} \subset\left\{\lambda \in \mathbb{C} ; \operatorname{Re} \lambda \leqslant-\delta_{0} \tilde{\eta}\left(\eta_{0}-\tilde{\eta}\right)\left(c-c_{*}\right)<0\right\}$. 
Proof of Theorem 2. The perturbation $u_{0}$ evolves in time as the solution to

$$
u_{t}=\mathcal{L} u+\mathcal{G}(u), \quad u_{\mid t=0}=u_{0},
$$

with $\mathcal{L}$ given by (3.9) and

$$
\mathcal{G}(u)=f(q+u)-f(q)-f^{\prime}(q) u .
$$

Note that $\mathcal{G}: \mathcal{X}_{\eta}^{2} \rightarrow \mathcal{X}_{\eta}^{2}$ is smooth since $f$ is smooth and $\mathcal{X}_{\eta}^{2}$ embeds continuously into the bounded continuous functions of the plane. Moreover, $\mathcal{G}(u)=\mathrm{O}\left(|u|^{2}\right)$ in $\mathcal{X}_{\eta}^{2}$. Given the spectral result in Proposition 3.2, the convergence estimate (3.8) is a simple consequence of the variation of constant formula for the solution $u$ to (3.10) and the contraction mapping principle.

Proof of Proposition 3.2. It is straightforward to check that $\mathcal{L}$ is sectorial, so it is sufficient to consider spectral values $\lambda$ in a bounded subset of the complex plane.

We write $\varepsilon^{2}=c-c_{*}>0$. Note that we can express the solution $q(x, y ; c)$ alternatively as a smooth function of $\varepsilon$, which we denote by $q(x, y ; \varepsilon)$, slightly abusing notation.

We have to solve

$$
D\left(\partial_{x x} u+\partial_{y y} u\right)+f^{\prime}(q(x, y ; c)) u+c \partial_{y} u-\lambda u=h(x, y),
$$

with bound

$$
|u|_{\mathcal{X}_{\eta}} \leqslant C(\lambda)|h|_{\mathcal{X}_{\eta}},
$$

for all $\lambda$ with $\operatorname{Re} \lambda \geqslant 0$, and sufficiently small weights $\eta \neq 0$. We therefore rewrite (3.12) as a first order system in the "evolution" variable $x$

$$
\begin{aligned}
& u_{x}=v, \\
& v_{x}=-\partial_{y y} u-D^{-1}\left(f^{\prime}(q(x, y ; c)) u+c \partial_{y} u-\lambda u\right)+D^{-1} h(x, y) .
\end{aligned}
$$

Set

$$
\tilde{q}^{\xi}(x, y ; \varepsilon)=\tilde{q}(x, y+\xi(x) ; \varepsilon):=q(x, y ; \varepsilon), \quad \tilde{h}^{\xi}(x, y):=D^{-1} h(x, y) .
$$

We have

$$
|\tilde{h}|_{\mathcal{X}_{\eta}} \leqslant C|h|_{\mathcal{X}_{\eta}}
$$

such that it is sufficient to bound the solution $u$ to (3.14) in terms of $\tilde{h}$. The eigenvalue problem we have to consider has been transformed to

$$
\begin{aligned}
& u_{x}=v, \\
& v_{x}=-\partial_{y y} u-D^{-1}\left(f^{\prime}\left(\tilde{q}^{\xi}(x, y ; \varepsilon)\right) u+c \partial_{y} u-\lambda u\right)+\tilde{h}^{\xi}(x, y) .
\end{aligned}
$$

Note that

$$
\tilde{q}^{\xi}(x, y ; \varepsilon)=q_{*}^{\xi}(y)+\mathrm{O}\left(\varepsilon^{2}\right),
$$

uniformly in $x, y$; see Theorem 1 . Then the linearization can be written as

$$
\begin{aligned}
& u_{x}=v, \\
& v_{x}=-\partial_{y y} u-D^{-1}\left(f^{\prime}\left(q_{*}^{\xi}(y)\right) u+c \partial_{y} u-\lambda u\right)+r^{\xi}(x, y) u+\tilde{h}^{\xi}(x, y),
\end{aligned}
$$

with $r^{\xi}=\mathrm{O}\left(\varepsilon^{2}\right)$. We write (3.18) in the abstract form

$$
\boldsymbol{u}_{x}=\mathcal{A}_{\lambda, \varepsilon}^{\xi}(x) \boldsymbol{u}+\boldsymbol{r}^{\xi}(x) \boldsymbol{u}+\tilde{\boldsymbol{h}}^{\xi}(x),
$$


on the Hilbert space $Y$ defined in the proof of Theorem 1.

Let us first consider the case $|\lambda| \geqslant \delta>0, \operatorname{Re} \lambda \geqslant 0$. Upon substituting $\boldsymbol{u}=\boldsymbol{v}^{\xi}$ into (3.19), we arrive at

$$
\boldsymbol{v}_{x}+\xi_{x} \partial_{y} \boldsymbol{v}=\mathcal{A}_{\lambda, \varepsilon}^{0} \boldsymbol{v}+\boldsymbol{r}^{0}(x) \boldsymbol{v}+\tilde{\boldsymbol{h}}(x),
$$

where we shifted back the equation by the $x$-dependent $y$-shift $\xi(\cdot)$. This equation is a small relatively bounded perturbation of the equation

$$
\boldsymbol{v}_{x}=\mathcal{A}_{\lambda, 0}^{0} \boldsymbol{v}+\tilde{\boldsymbol{h}}(x),
$$

where

$$
\mathcal{A}_{\lambda, 0}^{0}=\left(\begin{array}{cc}
0 & \mathrm{id} \\
-\partial_{y y}-D^{-1}\left(f^{\prime}\left(q_{*}(\cdot)\right)+c_{*} \partial_{y}-\lambda\right) & 0
\end{array}\right) .
$$

This equation can readily be solved by using the Fourier transform with respect to $x$, for any $\tilde{\boldsymbol{h}} \in L^{2}(\mathbb{R}, Y)$, and any $|\lambda| \geqslant \delta>0, \operatorname{Re} \lambda \geqslant 0$. We obtain the uniform bounds on the solution

$$
|\boldsymbol{v}|_{H^{1}(\mathbb{R}, Y)}+|\boldsymbol{v}|_{L^{2}\left(\mathbb{R}, Y^{1}\right)} \leqslant C|\tilde{\boldsymbol{h}}|_{L^{2}(\mathbb{R}, Y)} .
$$

Note that it is here that we need the strengthened stability Hypothesis 2.3 instead of the weaker zero stability Hypothesis 2.2, which was sufficient for the existence theorem. In particular, this hypothesis guarantees that $\mathcal{A}_{\lambda, 0}^{0}-\mathrm{i} k$ is invertible for all $k \in \mathbb{R}$ and any $\lambda \neq 0, \operatorname{Re} \lambda \geqslant 0$, since the problem $\left(\mathcal{A}_{\lambda, 0}^{0}-\mathrm{i} k\right)\left(v_{1}, v_{2}\right)^{T}=\left(h_{1}, h_{2}\right)^{T}$ has a unique solution

$$
\begin{aligned}
& v_{1}=-\left(\mathcal{M}_{k}-\lambda\right)^{-1} D\left(\mathrm{i} k h_{1}+h_{2}\right) \in H^{2}\left(\mathbb{R}, \mathbb{R}^{N}\right), \\
& v_{2}=\mathrm{i} k v_{1}-h_{1} \in H^{1}\left(\mathbb{R}, \mathbb{R}^{N}\right)
\end{aligned}
$$

for $\left(h_{1}, h_{2}\right) \in Y$. Standard perturbation theory then shows that the same bounds (3.21) hold for small relatively bounded perturbations and for the weighted spaces $L_{\eta}^{2}(\mathbb{R}, Y), H_{\eta}^{1}(\mathbb{R}, Y)$, and $L_{\eta}^{2}\left(\mathbb{R}, Y^{1}\right)$, with $\eta \neq 0$ small, where $L_{\eta}^{2}$ is defined similarly to (2.32) with norm $|\boldsymbol{w}|_{L_{\eta}^{2}}:=|\boldsymbol{w} / \cosh (\eta \cdot)|_{L^{2}}$. Therefore the estimates hold for the solutions $\boldsymbol{v}$ to (3.20), as well. Going back the change of variables to the $\boldsymbol{u}$-coordinates, we note that the norms in $\mathcal{X}_{\eta}$ are invariant under the $x$-dependent $y$-shift such that we have found a uniform bound on $u$ in $\mathcal{X}_{\eta}$ in terms of the norm of $h$ in $\mathcal{X}_{\eta}$. This proves the proposition in the parameter regime $|\lambda| \geqslant \delta$, for any fixed $\delta$ sufficiently small.

Consider $|\lambda| \ll 1$ in (3.19), next. We decompose

$$
\boldsymbol{u}=\sigma \boldsymbol{e}_{0}^{\xi}+\tau \boldsymbol{e}_{1}^{\xi}+\boldsymbol{w}^{\xi}, \quad \text { with } P^{\xi} \boldsymbol{w}^{\xi}=0 .
$$

Here, $\sigma$ and $\tau$ are real functions depending upon $x$, and the eigenvectors $\boldsymbol{e}_{j}^{\xi}$ and the projection $P^{\xi}$ have been defined in the proof of Theorem 1. Upon substituting this decomposition into (3.18), we find

$$
\begin{aligned}
& \sigma_{x} \boldsymbol{e}_{0}^{\xi}+\sigma \xi_{x}\left(\partial_{\xi} \boldsymbol{e}_{0}^{\xi}\right)+\tau_{x} \boldsymbol{e}_{1}^{\xi}+\tau \xi_{x}\left(\partial_{\xi} \boldsymbol{e}_{1}^{\xi}\right)+\left(\boldsymbol{w}^{\xi}\right)_{x} \\
& \quad=\tau \boldsymbol{e}_{0}^{\xi}+\mathcal{A}_{0,0}^{\xi} \boldsymbol{w}^{\xi}+\left(\mathcal{A}_{\lambda, \varepsilon}^{\xi}(x)-\mathcal{A}_{0,0}^{\xi}+\boldsymbol{r}^{\xi}(x)\right)\left(\sigma \boldsymbol{e}_{0}^{\xi}+\boldsymbol{w}^{\xi}\right)+\tilde{\boldsymbol{h}}^{\xi}(x),
\end{aligned}
$$

where we have used the fact that the first component of $\boldsymbol{e}_{1}^{\xi}$ vanishes.

We take the scalar product of (3.23) with $e_{1}^{\text {ad, } \xi}$ and $\boldsymbol{e}_{0}^{\text {ad, }, \xi}$, and project onto the hyperbolic part with (id $-P^{\xi}$ ). We find the equivalent system

$$
\begin{aligned}
& \sigma_{x}=\tau-\frac{c_{*}}{\lambda_{\mathrm{d}}^{\prime \prime}(0)} \xi^{\prime} \sigma+\mathrm{O}\left(|\varepsilon||\boldsymbol{w}|_{Y}\right), \\
& \tau_{x}=-\frac{c_{*}}{\lambda_{\mathrm{d}}^{\prime \prime}(0)} \xi^{\prime} \tau-\frac{2}{\lambda_{\mathrm{d}}^{\prime \prime}(0)} \lambda \sigma+\mathrm{O}\left(|\varepsilon|^{2}|\sigma|+(|\varepsilon|+|\lambda|)|\boldsymbol{w}|_{Y}\right)+h_{\tau}, \\
& \boldsymbol{w}_{x}=\mathcal{A}_{0,0}^{0} \boldsymbol{w}+\mathrm{O}\left((|\varepsilon|+|\lambda|)|\sigma|+|\varepsilon||\tau|+(|\varepsilon|+|\lambda|)|\boldsymbol{w}|_{Y^{1}}\right)+\boldsymbol{h}_{w}
\end{aligned}
$$


in which we shifted back the equation for $\boldsymbol{w}^{\xi}$ by the $x$-dependent $y$-shift $\xi(\cdot)$, and used the invariance of the norm in $Y$ under this shift. The error terms stand for bounded linear operators acting on $\sigma, \tau$, and $\boldsymbol{w}$ with bounds as indicated, and the functions $h_{\tau}$ and $\boldsymbol{h}_{w}$ belong to $L_{\eta}^{2}(\mathbb{R})$ and $L_{\eta}^{2}(\mathbb{R}, Y)$, respectively, with $L_{\eta}^{2}$-norms bounded by $C|h| \mathcal{X}_{\eta}$.

We set $\boldsymbol{w}_{0}=(\sigma, \tau), \mu=(\varepsilon, \lambda)$, and rewrite this system in the abstract form

$$
\begin{aligned}
& \boldsymbol{w}_{0 x}=L_{0} \boldsymbol{w}_{0}+\mu L_{00}(x) \boldsymbol{w}_{0}+\mu L_{01}(x) \boldsymbol{w}+\boldsymbol{h}_{0}(x), \\
& \boldsymbol{w}_{x}=\mathcal{A}_{0,0}^{0} \boldsymbol{w}+\mu L_{10}(x) \boldsymbol{w}_{0}+\mu L_{11}(x) \boldsymbol{w}+\boldsymbol{h}_{1}(x),
\end{aligned}
$$

where

$$
L_{0}=\left(\begin{array}{ll}
0 & 1 \\
0 & 0
\end{array}\right)
$$

$L_{i j}$ are $\mathrm{O}(1)$-bounded linear operators, and $\boldsymbol{h}_{0}=\left(0, h_{\tau}\right)^{T}, \boldsymbol{h}_{1}=\boldsymbol{h}_{w}$.

For $\boldsymbol{h}_{0} \equiv 0$ and $\boldsymbol{h}_{1} \equiv 0$ we have a nonautonomous, linear, homogeneous system. A center manifold reduction then shows that for small $\mu$ bounded solutions $\left(\boldsymbol{w}_{0}(x), \boldsymbol{w}(x)\right)$ to (3.27), (3.28) with $\boldsymbol{h}_{0} \equiv 0$ and $\boldsymbol{h}_{1} \equiv 0$ are of the form

$$
\left(\boldsymbol{w}_{0}(x), \boldsymbol{w}(x)=\phi(x ; \mu) \boldsymbol{w}_{0}(x)\right),
$$

with $\phi(x ; \mu): \mathbb{R}^{2} \rightarrow(\mathrm{id}-P) Y^{1}$ bounded linear operators with norms $|\phi(x ; \mu)|_{\mathcal{L}\left(\mathbb{R}^{2}, Y^{1}\right)} \leqslant C|\mu|$, for any $x \in \mathbb{R}$ and $\mu$ small; the dependence on $x$ and $\mu$ is $C^{k}$ for any finite $k$. Note that the hyperbolic part $\boldsymbol{w}$ of the solutions depends linearly upon $\boldsymbol{w}_{0}$, since the system is linear, and that the operators $\phi$ satisfy

$$
\begin{aligned}
\phi_{x}(x ; \mu)= & \mathcal{A}_{0,0}^{0} \phi(x ; \mu)-\phi(x ; \mu) L_{0}+\mu L_{10}(x)+\mu L_{11}(x) \phi(x ; \mu) \\
& -\mu \phi(x ; \mu) L_{00}(x)-\mu \phi(x ; \mu) L_{01}(x) \phi(x ; \mu),
\end{aligned}
$$

for any $x$ and $\mu$.

We now use the operators $\phi(x ; \mu)$ to construct bounded solutions to the inhomogeneous system (3.27), (3.28). We set

$$
\boldsymbol{w}(x)=\phi(x ; \mu) \boldsymbol{w}_{0}(x)+H(x) .
$$

Substituting (3.30) into (3.27), (3.28) and using (3.29) we find that the function $H$ solves the linear equation

$$
H^{\prime}(x)=\mathcal{A}_{0,0}^{0} H(x)+\mu L_{11}(x) H(x)-\mu \phi(x ; \mu) L_{01}(x) H(x)-\phi(x ; \mu) \boldsymbol{h}_{0}(x)+\boldsymbol{h}_{1}(x),
$$

and that the central part $\boldsymbol{w}_{0}$ satisfies the reduced system

$$
\boldsymbol{w}_{0 x}=L_{0} \boldsymbol{w}_{0}+\mu L_{00}(x) \boldsymbol{w}_{0}+\mu L_{01}(x) \phi(x ; \mu) \boldsymbol{w}_{0}+\mu L_{01} H(x)+\boldsymbol{h}_{0}(x) .
$$

Since (id $-P) \mathcal{A}_{0,0}^{0}-\mathrm{i} k$ is invertible on $\operatorname{Ker} P$ for all $k \in \mathbb{R}$, a standard perturbation argument shows that (3.31) has a unique solution $H(x)=H\left[\boldsymbol{h}_{0}, \boldsymbol{h}_{1}\right](x) \in \operatorname{Ker} P$ with bounds

$$
|H|_{H_{\eta}^{1}(\mathbb{R}, Y)}+|H|_{L_{\eta}^{2}\left(\mathbb{R}, Y^{1}\right)} \leqslant C\left(\left|\boldsymbol{h}_{0}\right|_{L_{\eta}^{2}\left(\mathbb{R}, \mathbb{R}^{2}\right)}+\left|\boldsymbol{h}_{1}\right|_{L_{\eta}^{2}(\mathbb{R}, Y)}\right) \leqslant C|h|_{\mathcal{X}_{\eta}} .
$$

Going back to the system (3.24)-(3.26) we find

$$
\boldsymbol{w}(x)=\phi(x ; \varepsilon, \lambda)(\sigma(x), \tau(x))+H(x),
$$

with $H$ as above, and

$$
|\phi(x ; \varepsilon, \lambda)(\sigma(x), \tau(x))|_{Y^{1}} \leqslant C(|\varepsilon|+|\lambda|)(|\sigma(x)|+|\tau(x)|) .
$$

Substitution of (3.32) into (3.24), (3.25) gives 


$$
\begin{aligned}
& \sigma_{x}+\frac{c_{*}}{\lambda_{\mathrm{d}}^{\prime \prime}(0)} \xi^{\prime} \sigma=\tau+\mathrm{O}\left(\left(|\varepsilon|^{2}+|\varepsilon \lambda|\right)(|\sigma|+|\tau|)\right)+\tilde{h}_{\sigma}(x), \\
& \tau_{x}+\frac{c_{*}}{\lambda_{\mathrm{d}}^{\prime \prime}(0)} \xi^{\prime} \tau=\alpha_{\varepsilon} \varepsilon^{2} \sigma-\frac{2}{\lambda_{\mathrm{d}}^{\prime \prime}(0)} \lambda \sigma+\mathrm{O}\left(\left(|\varepsilon \lambda|+|\lambda|^{2}\right)|\sigma|+(|\varepsilon|+|\lambda|)^{2}|\tau|\right)+\tilde{h}_{\tau}(x) .
\end{aligned}
$$

Here, $\alpha_{\varepsilon} \in \mathbb{R}$, and $\tilde{h}_{\sigma} \in H_{\eta}^{1}(\mathbb{R})$ and $\tilde{h}_{\tau} \in L_{\eta}^{2}(\mathbb{R})$ with norm bounded by $C|h|_{\mathcal{X}_{\eta}}$.

Note that for $\lambda=0$, and $h \equiv 0$ the $y$-derivative of the corner is a solution of (3.12). The corresponding solution to (3.33), (3.34) is

$$
\sigma=1+\mathrm{O}\left(\varepsilon^{2}\right), \quad \tau=\mathrm{O}(\varepsilon)
$$

and therefore $\sigma \equiv 1$ necessarily solves the truncated equation

$$
\begin{aligned}
\sigma_{x}+\frac{c_{*}}{\lambda_{\mathrm{d}}^{\prime \prime}(0)} \xi^{\prime} \sigma & =\tau, \\
\tau_{x}+\frac{c_{*}}{\lambda_{\mathrm{d}}^{\prime \prime}(0)} \xi^{\prime} \tau & =\alpha_{\varepsilon} \varepsilon^{2} \sigma .
\end{aligned}
$$

This gives

$$
\frac{c_{*}}{\lambda_{\mathrm{d}}^{\prime \prime}(0)} \xi^{\prime \prime}+\left(\frac{c_{*} \xi^{\prime}}{\lambda_{\mathrm{d}}^{\prime \prime}(0)}\right)^{2}-\alpha_{\varepsilon} \varepsilon^{2} \equiv 0,
$$

from which we compute the constant $\alpha_{\varepsilon}$ by the help of (2.30), and find

$$
\alpha_{\varepsilon}=\frac{2 c_{*}}{\left(\lambda_{d}^{\prime \prime}(0)\right)^{2}} .
$$

In order to prove the proposition, it is now sufficient to show that the homogeneous equation corresponding to (3.33), (3.34) possesses an exponential dichotomy relative to the exponential weight $\eta$ for all $\lambda$ and $\varepsilon$ sufficiently small.

The natural scaling of (3.35), (3.36) predicts $\lambda=\tilde{\lambda} \varepsilon^{2}$ and $\tilde{\lambda}$ bounded. Let us justify this scaling. Suppose therefore that $\varepsilon^{2}=\delta^{2}|\lambda|$ with $\delta$ small. Substituting the scaling into the homogeneous part of (3.33), (3.34), and scaling $\zeta=|\lambda|^{1 / 2} x, \tau=|\lambda|^{1 / 2} \tilde{\tau}$ we find

$$
\begin{aligned}
& \sigma_{\zeta}=\tilde{\tau}+\mathrm{O}(|\delta|), \\
& \tilde{\tau}_{\zeta}=-\frac{2}{\lambda_{\mathrm{d}}^{\prime \prime}(0)} \mathrm{e}^{\mathrm{i} \arg (\lambda)} \sigma+\mathrm{O}(|\delta|+|\lambda|)
\end{aligned}
$$

so that

$$
\sigma_{\zeta \zeta}+\frac{2}{\lambda_{d}^{\prime \prime}(0)} \mathrm{e}^{\mathrm{i} \arg (\lambda)} \sigma=\mathrm{O}(|\delta|+|\lambda|) .
$$

Since in $|\lambda|=0$ and $\delta=0$, this equation possesses an exponential dichotomy, a robustness result gives invertibility of the linearization outside the "natural" scaling. We therefore scale $\lambda=\tilde{\lambda} \varepsilon^{2}$ with $\tilde{\lambda}$ bounded, $\zeta=\varepsilon x, \tau=\varepsilon \tilde{\tau}$, and we compute

$$
\begin{aligned}
& \sigma_{\zeta}+\frac{c_{*}}{\lambda_{\mathrm{d}}^{\prime \prime}(0)} \frac{\xi^{\prime}}{\varepsilon} \sigma=\tilde{\tau}+\mathrm{O}(|\varepsilon|) \\
& \tilde{\tau}_{\zeta}+\frac{c_{*}}{\lambda_{\mathrm{d}}^{\prime \prime}(0)} \frac{\xi^{\prime}}{\varepsilon} \tilde{\tau}=\alpha_{\varepsilon}-\frac{2}{\lambda_{\mathrm{d}}^{\prime \prime}(0)} \tilde{\lambda} \sigma+\mathrm{O}(|\varepsilon|),
\end{aligned}
$$

so that

$$
\sigma_{\zeta \zeta}+2 \frac{c_{*}}{\lambda_{\mathrm{d}}^{\prime \prime}(0)} \frac{\xi^{\prime}}{\varepsilon} \sigma_{\zeta}+\frac{2}{\lambda_{\mathrm{d}}^{\prime \prime}(0)} \tilde{\lambda} \sigma=\mathrm{O}(\varepsilon)
$$


In the limit $\varepsilon=0$, we find the linearization about the Burgers shock in the integrated form

$$
\sigma_{\zeta \zeta}+2 \frac{\sqrt{2 c_{*}}}{\lambda_{\mathrm{d}}^{\prime \prime}(0)} \tanh \left(\frac{\sqrt{2 c_{*}}}{\lambda_{\mathrm{d}}^{\prime \prime}(0)} \zeta\right) \sigma_{\zeta}+\frac{2}{\lambda_{\mathrm{d}}^{\prime \prime}(0)} \tilde{\lambda} \sigma=0 ;
$$

see (3.5), (3.6). Since the spectrum of this equation is contained in the region

$$
\operatorname{Re} \tilde{\lambda} \leqslant \frac{\lambda_{\mathrm{d}}^{\prime \prime}(0)}{2} \tilde{\eta}\left(-2 \frac{\sqrt{2 c_{*}}}{\lambda_{\mathrm{d}}^{\prime \prime}(0)}-\tilde{\eta}\right)<0,
$$

for any positive weight $\tilde{\eta} \in\left(0,-2 \sqrt{2 c_{*}} / \lambda_{\mathrm{d}}^{\prime \prime}(0)\right)$, a standard perturbation argument concludes the proof of the proposition.

Remark 3.3. The proof of Proposition 3.2 actually gives much more insight into spectral properties of the linearization. To leading order, we found that the extended point spectrum is trivial and the dispersion relation to the essential spectrum possesses a quadratic expansion; see [16,37,50] for background on extensions of point spectra and Evans function into the essential spectrum. We expect that stability results in spaces with algebraic weights, similar to the ones in [33,35], hold for the weak interior corners, constructed in the present paper; see also [12].

Proposition 3.4. Under the assumptions of Theorem 2, there exist positive constants $\varepsilon_{0}, \eta_{0}$ and $\delta_{0}$ such that for any $c-c_{*} \in\left(0, \varepsilon_{0}\right)$, and any weight $\eta=\tilde{\eta}\left(c-c_{*}\right)^{1 / 2}$ with $\tilde{\eta} \in\left(0, \eta_{0}\right)$, the spectrum of $\tilde{\mathcal{L}}$ in $\tilde{\mathcal{X}}_{\eta}$ satisfies

$$
\operatorname{spec} \tilde{\mathcal{L}} \cap\left\{\lambda \in \mathbb{C} ; \operatorname{Re} \lambda>-\delta_{0} \tilde{\eta}\left(\eta_{0}-\tilde{\eta}\right)\left(c-c_{*}\right)\right\}=\{0\},
$$

with $\lambda=0$ being an isolated eigenvalue of geometric and algebraic multiplicity two.

Proof of Theorem 3. The proof is similar to the proof of Theorem 2. Note that the nonlinearity $\mathcal{G}$ as defined in (3.11) smoothly maps $\tilde{\mathcal{X}}_{\eta}^{2}$ into itself. Also note that the family of translates $q\left(x+x_{0}, y+y_{0} ; c\right)$ generates a plane of equilibria to (3.10) in the function space $\tilde{\mathcal{X}}_{\eta}^{2}$. The statement of the theorem is therefore a simple consequence of the stable manifold theorem [27].

Proof of Proposition 3.4. The operator $\mathcal{L}$ is sectorial on $\tilde{\mathcal{X}}_{\eta}$ with domain of definition $\tilde{\mathcal{X}}_{\eta}^{2}$, so it is sufficient to consider spectral values $\lambda$ in a bounded subset of the complex plane. We have to solve

$$
D\left(\left(\partial_{x x}+\xi_{x} \partial_{y y}\right)^{2} \tilde{u}+\partial_{y y} \tilde{u}\right)+c \partial_{y} \tilde{u}+f^{\prime}(\tilde{q}(\cdot, \cdot ; c)) \tilde{u}-\lambda \tilde{u}=\tilde{h}(x, y),
$$

with bound

$$
|\tilde{u}|_{\tilde{\mathcal{X}}_{\eta}} \leqslant C(\lambda)|\tilde{h}|_{\tilde{\mathcal{X}}_{\eta}}
$$

for all $\lambda$ with $\operatorname{Re} \lambda \geqslant 0$, and sufficiently small weights $\eta \neq 0$. Set $\varepsilon^{2}=c-c_{*}>0$, so that $\tilde{q}(\cdot, \cdot ; c)=q_{*}(\cdot)+\mathrm{O}\left(\varepsilon^{2}\right)$. Since on $\mathcal{X}_{\eta}$ this eigenvalue problem is equivalent to the one for $\mathcal{L}$ it is enough to take $\tilde{h} \in \mathcal{X}_{\text {step. }}$. As in the proof of Proposition 3.2 we write the eigenvalue problem as a first order system in the "evolution" variable $x$

$$
\begin{aligned}
& \tilde{u}_{x}+\xi_{x} \tilde{u}_{y}=\tilde{v}, \\
& \tilde{v}_{x}+\xi_{x} \tilde{v}_{y}=-\partial_{y y} \tilde{u}-D^{-1}\left(f^{\prime}\left(q_{*}(\cdot)\right) \tilde{u}+c \partial_{y} \tilde{u}-\lambda \tilde{u}\right)+r^{0}(x, y) \tilde{u}+\tilde{h},
\end{aligned}
$$

with $r^{0}=\mathrm{O}\left(\varepsilon^{2}\right)$, or in the abstract form

$$
\boldsymbol{v}_{x}+\xi_{x} \partial_{y} \boldsymbol{v}=\mathcal{A}_{\lambda, \varepsilon}^{0}(x) \boldsymbol{v}+\boldsymbol{r}^{0}(x) \boldsymbol{v}+\tilde{\boldsymbol{h}}(x)
$$

see also (3.20). We introduce the function space

$$
L_{\text {step }}(\mathbb{R}, Y)=\left\{\boldsymbol{v}(x, y)=\boldsymbol{v}_{-}(y)(1-\theta(x))+\boldsymbol{v}_{+}(y) \theta(x) ; \boldsymbol{v}_{ \pm} \in Y\right\},
$$


and similarly $L_{\text {step }}\left(\mathbb{R}, Y^{1}\right)$. Then it is enough to find a solution $\boldsymbol{v} \in L_{\eta}^{2}\left(\mathbb{R}, Y^{1}\right) \cap L_{\text {step }}\left(\mathbb{R}, Y^{1}\right)$ to (3.39), for $\tilde{\boldsymbol{h}} \in$ $L_{\text {step }}(\mathbb{R}, Y)$.

For $|\lambda| \geqslant \delta>0, \operatorname{Re} \lambda \geqslant 0$ we solve (3.39) as in Proposition 3.4 by using standard perturbation theory after solving the problem at $\varepsilon=0$,

$$
\boldsymbol{v}_{x}=\mathcal{A}_{\lambda, 0}^{0} \boldsymbol{v}+\tilde{\boldsymbol{h}}(x),
$$

for $\tilde{\boldsymbol{h}} \in L_{\text {step }}(\mathbb{R}, Y)$, and weight $\eta=0$. In order to solve this equation we write

$$
\tilde{\boldsymbol{h}}(x, y)=\tilde{\boldsymbol{h}}_{c}(y)+\tilde{\boldsymbol{h}}_{s}(y)(2 \theta(x)-1),
$$

and solve (3.40) for $\tilde{\boldsymbol{h}}_{c}$ and $\tilde{\boldsymbol{h}}_{s}(2 \theta-1)$ separately. For the $x$-independent part $\tilde{\boldsymbol{h}}_{c}$ the solution is given by

$$
\boldsymbol{v}_{c}=\left(\mathcal{A}_{\lambda, 0}^{0}\right)^{-1} \tilde{\boldsymbol{h}}_{c} \in Y^{1}
$$

since $\mathcal{A}_{\lambda, 0}^{0}$ is invertible. For the second term we use the Fourier transform in $x$ and obtain a solution $\boldsymbol{v}_{s}=\left(v_{1}, v_{2}\right)$ given by

$$
\begin{aligned}
& v_{1}(x)=-\sqrt{\frac{2}{\pi}} \mathcal{F}^{-1}\left[\left(\mathcal{M}_{k}-\lambda\right)^{-1} D\left(h_{s 1}+\frac{1}{\mathrm{i} k} h_{s 2}\right)\right], \\
& v_{2}(x)=-\sqrt{\frac{2}{\pi}} \mathcal{F}^{-1}\left[\left(\mathcal{M}_{k}-\lambda\right)^{-1} D\left(\mathrm{i} k h_{s 1}+h_{s 2}\right)\right]-h_{s 1}
\end{aligned}
$$

where $\tilde{\boldsymbol{h}}_{s}=\left(h_{s 1}, h_{s 2}\right)$, and $\mathcal{F}^{-1}$ denotes the inverse Fourier transform. We write

$$
\begin{aligned}
v_{1}(x)= & -\left(\mathcal{M}_{0}-\lambda\right)^{-1}\left(D h_{s 2}\right)(2 \theta(x)-1) \\
& -\sqrt{\frac{2}{\pi}} \mathcal{F}^{-1}\left[\left(\mathcal{M}_{k}-\lambda\right)^{-1} D h_{s 1}+\frac{1}{\mathrm{i} k}\left(\left(\mathcal{M}_{k}-\lambda\right)^{-1}-\left(\mathcal{M}_{0}-\lambda\right)^{-1}\right) D h_{s 2}\right] .
\end{aligned}
$$

Then the first term in the right-hand side of this equality belongs to $L_{\text {step }}\left(\mathbb{R}, H^{2}\left(\mathbb{R}, \mathbb{R}^{N}\right)\right)$, and it is straightforward to check that the second term belongs to $L^{2}\left(\mathbb{R}, H^{2}\left(\mathbb{R}, \mathbb{R}^{N}\right)\right)$. Similarly we find that $v_{2} \in L_{\text {step }}\left(\mathbb{R}, H^{1}\left(\mathbb{R}, \mathbb{R}^{N}\right)\right) \oplus$ $L^{2}\left(\mathbb{R}, H^{1}\left(\mathbb{R}, \mathbb{R}^{N}\right)\right)$, so that $\boldsymbol{v} \in L_{\text {step }}\left(\mathbb{R}, Y^{1}\right) \oplus L^{2}\left(\mathbb{R}, Y^{1}\right)$. This proofs the result in the Proposition for $|\lambda| \geqslant \delta>0$.

For $|\lambda| \ll 1$ the proof follows verbatim the proof of Proposition 3.2. After justifying the scaling, we find to leading order the eigenvalue problem for the eikonal equation in the space of exponentially decreasing functions, augmented by arbitrary step functions. Recall that the reduced linearized operator is Fredholm with index zero and actually invertible when restricted to the subspace of exponentially decreasing functions. In the full space, augmented with the step functions $\theta(x)$ and $(1-\theta(x))$, the reduced operator possesses block-diagonal structure and therefore is Fredholm of index zero when augmented by a finite-dimensional operator. As a consequence, the spectrum of the linearization can be determined by finding the values of $\lambda$ such that the reduced problem possesses a kernel, i.e. we have to determine the bounded solutions to (3.37). For the truncated equation (3.38), given as the linearization about the shock solution in the eikonal equation, the only bounded solutions in $\operatorname{Re} \lambda \geqslant-\delta, \delta>0$ occur for $\lambda=0$ and are given by

$$
\sigma_{1}(\zeta)=1, \quad \sigma_{2}(\zeta)=\tanh \left(\frac{\sqrt{2 c_{*}}}{\lambda_{\mathrm{d}}^{\prime \prime}(0)} \zeta\right)
$$

Therefore, zero is an algebraically and geometrically double eigenvalue which persists under the perturbation, as the unique eigenvalue in $\operatorname{Re} \lambda \geqslant-\delta$, with eigenfunctions given by the translational derivatives of the corner, $\partial_{y} q(x, y ; c)$ and $\partial_{x} q(x, y ; c)$, respectively. This concludes the proof of Proposition 3.4.

Remark 3.5. It seems natural to extend the function space further to include rotations of the corner defect, with linear growth of the difference in $x$. This would correspond to perturbations in Burgers' equation with infinite 
$L^{2}$-norm, allowing to change the asymptotic states of the shock. To our knowledge, stability of Burgers' shock with respect to these perturbation is not known. In our set-up, perturbations would select a different angle at $x=+\infty$ or $x=-\infty$, such that propagation would not occur in the $y$-direction any more.

\section{Defects generated by transverse instabilities}

In this section we replace the Hypotheses 2.2 and 2.3 on transverse stability of the planar travelling waves by an instability assumption, $\lambda_{d}^{\prime \prime}(0)>0$. Then the travelling waves can be stable in one space-dimension but they are unstable with respect to a band of wave numbers in the transverse direction. We discuss two scenarios, here. In the first case, a fully developed instability, $\lambda_{d}^{\prime \prime}(0)>0$, we show that exterior corners in (unstable) flat interfaces do exist to any order in an asymptotic expansion of the reduced system, but typically do not persist for the full system. For the full system, the interface of the corner defect looses its flatness at infinity, where small periodic modulations appear on either one or both sides of the corner. We then address the onset of instability $\lambda_{\mathrm{d}}^{\prime \prime}(0)=0$, driving the instability with a parameter $\mu$, such that $\partial_{\mu} \lambda_{d}^{\prime \prime}(0)>0$. To leading order, we recover the KuramotoSivashinsky equation, which has formally been derived in [59]; see also [65] for examples. Known results on existence of heteroclinic and homoclinic orbits for this equation permit to conclude existence of both interior and exterior corners, and of steps, for $\mu>0$ where the planar front is unstable.

\subsection{Fully developed instabilities}

We keep Hypothesis 2.1 on existence of planar travelling waves and replace Hypothesis 2.2 on zero-stability by the following assumption.

Hypothesis 4.1 (Instability). We assume that the spectrum of $\mathcal{L}_{*}$ on the negative real axis consists of exactly two isolated eigenvalues $\lambda=0$ and $\lambda=-k_{*}^{2}$ with algebraic multiplicity one.

This hypothesis is a consequence of the following assumption on transverse instability of the planar travelling waves.

Hypothesis 4.2 (Transverse instability). Assume that the travelling wave is asymptotically stable in one space dimension, that is, the essential spectrum of $\mathcal{M}_{0}$, as defined in (2.8), is strictly contained in the left half plane and zero is the only eigenvalue in the closed right half plane, with algebraic multiplicity one. Furthermore, assume that there is a $k_{*}>0$ such that the spectra of $\mathcal{M}_{k}$ are strictly contained in the left half plane for $|k|>k_{*}$, and that for $|k| \leqslant k_{*}$ there is exactly one spectral value of $\mathcal{M}_{k}$ in the closed right half plane, $\lambda_{\mathrm{d}}(k)$, which is a simple eigenvalue satisfying $\lambda_{\mathrm{d}}(0)=\lambda_{\mathrm{d}}^{\prime}(0)=\lambda_{\mathrm{d}}\left( \pm k_{*}\right)=0, \lambda_{\mathrm{d}}^{\prime \prime}(0)>0, \lambda_{\mathrm{d}}^{\prime}\left( \pm k_{*}\right) \neq 0$ and $\lambda_{\mathrm{d}}(k)>0$ for $0<|k|<k_{*}$; see Fig. 5 .
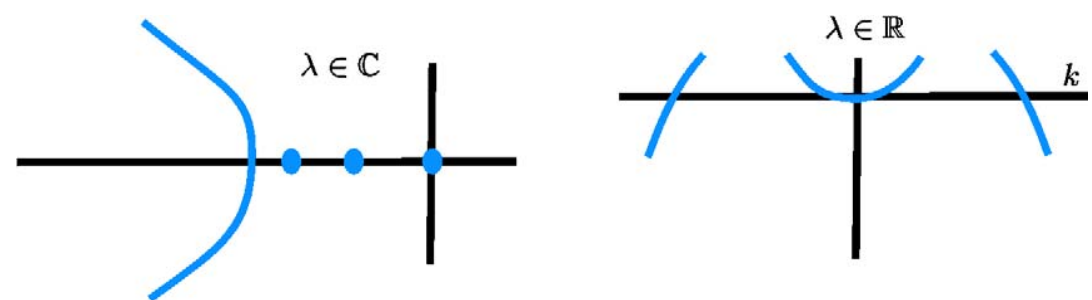

Fig. 5. To the left the spectrum of $\mathcal{M}_{0}$ and to the right the critical spectra of the $\mathcal{M}_{k}$ parameterized by $k$, in case of a fully developed transverse instability. 
Remark 4.3. The quadratic tangency of the dispersion relation $\lambda_{d}^{\prime \prime}(0)>0$ implies instability of the planar travelling wave with respect to transverse perturbations of wavelength $k,|k| \leqslant k_{*}$.

\section{Lemma 4.4. Hypothesis 4.2 implies that Hypothesis 4.1 on instability holds.}

The proof of this lemma is similar to the one of Lemma 2.5 and will be omitted.

We start from the formulation of the travelling-wave equation (2.2) as a dynamical system which was given in the proof of Theorem 1, Eqs. (2.16)-(2.18). From Hypothesis 4.1 we obtain that the spectrum of the linearization $\mathcal{A}_{*}$ about the planar travelling wave $\boldsymbol{q}_{*}^{0}$ satisfies

$$
\operatorname{spec} \mathcal{A}_{*} \cap\{|\operatorname{Re} \lambda| \leqslant \epsilon\}=\left\{0, \pm \mathrm{i} k_{*}\right\},
$$

in which the origin is an eigenvalue with algebraic multiplicity two and $\pm \mathrm{i} k_{*}$ are eigenvalues with algebraic multiplicity one.

The kernel and generalized kernel of $\mathcal{A}_{*}$, and of its adjoint $\mathcal{A}_{*}^{\text {ad }}$, coincide with the ones found in the proof of Theorem 1. In addition, we consider the kernel of $\mathcal{A}_{*} \mp \mathrm{i} k_{*}$,

$$
\operatorname{ker}\left(\mathcal{A}_{*} \mp \mathrm{i} k_{*}\right)=\operatorname{span}\left(\boldsymbol{e}_{ \pm}\right), \quad \boldsymbol{e}_{ \pm}=\left(\begin{array}{c}
r_{*}(\cdot) \\
\pm \mathrm{i} k_{*} r_{*}(\cdot)
\end{array}\right),
$$

where $r_{*}(\cdot)$ is the real-valued eigenvector associated to the eigenvalue $\lambda_{\mathrm{d}}\left(k_{*}\right)=0$ of $\mathcal{M}_{k_{*}}$. In particular, $\mathcal{L}_{*} r_{*}=$ $-k_{*}^{2} r_{*}$ and $\boldsymbol{e}_{-}=\overline{\boldsymbol{e}}_{+}$. For the adjoint $\mathcal{A}_{*}^{\text {ad }} \pm \mathrm{i} k_{*}$ we find

$$
\operatorname{ker}\left(\mathcal{A}_{*}^{\mathrm{ad}} \pm \mathrm{i} k_{*}\right)=\operatorname{span}\left(\boldsymbol{e}_{ \pm}^{\mathrm{ad}}\right), \quad \boldsymbol{e}_{ \pm}^{\mathrm{ad}}=\left(\begin{array}{c}
k_{*} r_{*}^{\mathrm{ad}}(\cdot) \\
\pm \mathrm{i} r_{*}^{\mathrm{ad}}(\cdot)
\end{array}\right),
$$

where $r_{*}^{\text {ad }}(\cdot)$ is the unique real-valued function satisfying $\mathcal{L}_{*}^{\mathrm{ad}} r_{*}^{\mathrm{ad}}=-k_{*}^{2} r_{*}^{\mathrm{ad}}$ and $\left(r_{*}^{\mathrm{ad}}, r_{*}\right)_{L^{2}}=1 / 2 k_{*}$, so that we have

$$
\left\langle\boldsymbol{e}_{ \pm}^{\mathrm{ad}}, \boldsymbol{e}_{ \pm}\right\rangle_{L^{2} \times L^{2}}=1, \quad\left\langle\boldsymbol{e}_{ \pm}^{\mathrm{ad}}, \boldsymbol{e}_{\mp}\right\rangle_{L^{2} \times L^{2}}=0
$$

The spectral projection $P: Y \rightarrow Y$ associated with the central eigenvalues 0 and $\pm \mathrm{i} k_{*}$ of $\mathcal{A}_{*}$ is given by

$$
P \boldsymbol{u}=\left\langle\boldsymbol{e}_{1}^{\mathrm{ad}}, \boldsymbol{u}\right\rangle_{L^{2} \times L^{2}} \boldsymbol{e}_{0}+\left\langle\boldsymbol{e}_{0}^{\mathrm{ad}}, \boldsymbol{u}\right\rangle_{L^{2} \times L^{2}} \boldsymbol{e}_{1}+\left\langle\boldsymbol{e}_{+}^{\mathrm{ad}}, \boldsymbol{u}\right\rangle_{L^{2} \times L^{2}} \boldsymbol{e}_{+}+\left\langle\boldsymbol{e}_{-}^{\mathrm{ad}}, \boldsymbol{u}\right\rangle_{L^{2} \times L^{2}} \boldsymbol{e}_{-} .
$$

Similarly, to the shifted equilibria $\boldsymbol{q}_{*}^{\xi}$ we introduce the shifted eigenvectors $\boldsymbol{e}_{ \pm}^{\xi}=\left(r_{*}^{\xi}, \pm \mathrm{i} k_{*} r_{*}^{\xi}\right)^{T}$, and analogously $\boldsymbol{e}_{ \pm}^{\mathrm{ad}, \xi}$ and $P^{\xi}$.

Following the reduction strategy in Section 2, we write

$$
\boldsymbol{u}=\boldsymbol{q}_{*}^{\xi}+\eta \boldsymbol{e}_{1}^{\xi}+A \boldsymbol{e}_{+}^{\xi}+\bar{A} \boldsymbol{e}_{-}^{\xi}+\boldsymbol{w}^{\xi}, \quad \text { with } P^{\xi} \boldsymbol{w}^{\xi}=P \boldsymbol{w}=0
$$

Here $\xi, \eta$ are real functions and $A$ is complex-valued function depending upon $x$. Substituting (4.1) into (2.17), and then taking successively the scalar product with $\boldsymbol{e}_{1}^{\mathrm{ad}, \xi}, \boldsymbol{e}_{0}^{\mathrm{ad}, \xi}, \boldsymbol{e}_{ \pm}^{\mathrm{ad}, \xi}$, and projecting with id $-P^{\xi}$ we find the equation for $\xi$,

$$
\xi_{x}=\eta+\mathrm{O}\left(|\eta|\left(|A|+|\boldsymbol{w}|_{Y}\right)\right)
$$

and the quasilinear system

$$
\begin{aligned}
& \eta_{x}=\frac{2}{\lambda_{\mathrm{d}}^{\prime \prime}(0)}\left(c-c_{*}\right)-\frac{c_{*}}{\lambda_{\mathrm{d}}^{\prime \prime}(0)} \eta^{2}+\mathrm{O}\left(\left|c-c_{*}\right|\left(|A|+|\boldsymbol{w}|_{Y}\right)+|\eta|\left(|A|+|\boldsymbol{w}|_{Y}\right)+\left(|A|+|\boldsymbol{w}|_{Y}\right)^{2}\right), \\
& A_{x}=\mathrm{i} k_{*} A+\mathrm{O}\left(\left|c-c_{*}\right|+(|\eta|+|A|)^{2}+|\boldsymbol{w}|_{Y}^{2}+|\eta||\boldsymbol{w}|_{Y^{1}}\right), \\
& \boldsymbol{w}_{x}=\mathcal{A}_{*} \boldsymbol{w}+\mathrm{O}\left(\left|c-c_{*}\right|+(|\eta|+|A|)^{2}+|\boldsymbol{w}|_{Y}^{2}+|\eta||\boldsymbol{w}|_{Y^{1}}\right),
\end{aligned}
$$


in which $\xi_{x}$ has been replaced by the expression in (4.2). The system (4.3)-(4.5) is posed on the Hilbert space $\mathbb{R} \times$ $\mathbb{C} \times Y_{h}$, in which $Y_{h}=(\mathrm{id}-P) Y$. Using again a center-manifold reduction for quasilinear systems [45, Theorem 1] we conclude that, for $c$ close to $c_{*}$, all solutions with $\eta, A, \boldsymbol{w}$ sufficiently small are solutions to a reduced system for $\eta$ and $A$, obtained by substituting $\boldsymbol{w}=h\left(\boldsymbol{\eta}, A ; c-c_{*}\right)=\mathrm{O}\left(\left|c-c_{*}\right|+(|\eta|+|A|)^{2}\right)$ into (4.3)-(4.4). If we further exploit that the reversibility acts on this reduced system as $\eta \mapsto-\eta$ and $A \mapsto \bar{A}$, we conclude that it has the form

$$
\begin{aligned}
& \eta_{x}=\frac{2}{\lambda_{\mathrm{d}}^{\prime \prime}(0)}\left(c-c_{*}\right)-\frac{c_{*}}{\lambda_{\mathrm{d}}^{\prime \prime}(0)} \eta^{2}+\mathrm{O}\left(\left|c-c_{*}\right|\left(\left|c-c_{*}\right|+|\eta|^{2}+|A|\right)+|\eta|^{4}+|\eta||A|+|A|^{2}\right), \\
& A_{x}=\mathrm{i} \alpha_{*}\left(c-c_{*}\right)+\mathrm{i} k_{*} A+\mathrm{O}\left(\left(\left|c-c_{*}\right|+|\eta|+|A|\right)^{2}\right),
\end{aligned}
$$

where $\alpha_{*}=\left(r_{*}^{\mathrm{ad}}, D^{-1} q_{*}^{\prime}\right) \in \mathbb{R}$.

The reduced system (4.6)-(4.7) has two equilibria

$$
\eta_{ \pm}=\mp \sqrt{2\left(c-c_{*}\right) / c_{*}}+\mathrm{O}\left(\left|c-c_{*}\right|\right), \quad A_{ \pm}=\mathrm{O}\left(\left|c-c_{*}\right|\right),
$$

corresponding to rotations of the planar front, just as in Section 2. Next, normal form theory (cf. e.g. [31,42]) shows that the reduced system can be transformed into

$$
\begin{aligned}
& \tilde{\eta}_{x}=P_{1}\left(\tilde{\eta}^{2},|\tilde{A}|^{2} ; c-c_{*}\right)+\mathrm{O}\left((|\tilde{\eta}|+|\tilde{A}|)^{2 k+1}\right), \\
& \tilde{A}_{x}=\mathrm{i} k_{*} \tilde{A}+\mathrm{i} \tilde{A} P_{2}\left(\tilde{\eta},|\tilde{A}|^{2} ; c-c_{*}\right)+\mathrm{O}\left((|\tilde{\eta}|+|\tilde{A}|)^{k+2}\right),
\end{aligned}
$$

by a polynomial change of variables

$$
\eta=\tilde{\eta}+Q_{1}\left(\tilde{\eta}, \tilde{A} ; c-c_{*}\right), \quad A=\tilde{A}+Q_{2}\left(\tilde{\eta}, \tilde{A} ; c-c_{*}\right),
$$

which preserves reversibility. Here $Q_{j}(0,0 ; 0)=D_{\eta} Q_{j}(0,0 ; 0)=D_{A} Q_{j}(0,0 ; 0)=0$, and similar equalities hold for $P_{j}$ which are polynomials in their first two arguments of degree $k$. In particular, $\tilde{A}=0$ is an invariant line to any order in the normal form. Along this line there is a heteroclinic orbit connecting the two equilibria $\left(\tilde{\eta}_{ \pm}, 0\right)$, obtained as the codimension-two intersection between the one-dimensional stable and unstable manifold of the equilibria. This heteroclinic connection would correspond to an exterior corner in the reaction-diffusion system. Nonnormal form perturbations typically break this connection; see [42]. However, both equilibria are surrounded by a family of periodic orbits, which persist due to reversibility by Lyapunov's center theorem. In particular, the stable manifold of the family of periodic orbits contains a full neighborhood of $\left(\tilde{\eta}_{+}, 0\right)$, such that the heteroclinic orbit in the normal form persists as a heteroclinic to one of the periodic orbits. Phenomenologically, these heteroclinic orbits correspond to exterior corners with a periodic modulation of the flat interface on either side of the corner. Similarly, symmetric exterior corners with periodic modulations on both sides of the corner exist. In both cases, we expect that the minimal amplitude of the periodic structures is exponentially small in the angle of the corner for analytic kinetics [42].

\subsection{The onset of instability}

Throughout this section, we consider the parameter-dependent system

$$
u_{t}=D \Delta_{x, y} u+c \partial_{y} u+f(u ; \mu),
$$

in which $\mu$ is a real parameter, and the corresponding stationary equation

$$
D \Delta_{x, y} u+c \partial_{y} u+f(u ; \mu)=0 .
$$

We assume the existence of a planar travelling wave connecting two homogeneous equilibria for $\mu=0$, Hypothesis 2.1. We replace Hypothesis 2.2 by the following assumption. 
Hypothesis 4.5 (Zero-stability). We assume that $\mathcal{L}_{*}-\lambda$ id is invertible for all $\lambda<0$ and that $\lambda=0$ is an isolated eigenvalue with geometric multiplicity one and algebraic multiplicity two.

Using Lyapunov-Schmidt reduction on the one-dimensional kernel of the linearization, it is straightforward to conclude that there exists a smooth family of fronts $q_{*}(y ; \mu)$ with speeds $c_{*}(\mu)$ connecting the asymptotic states $q_{ \pm}(\mu)$ for $\mu \sim 0$. Here, smoothness refers to the dependence on $y$ in $C_{\text {loc }}^{k}$. Notice that the $\mu$-dependence of the asymptotic states $q_{ \pm}(\mu)$ can be eliminated by an affine transformation. This will introduce an additional $\mu$-dependence in the coefficients of the reaction-diffusion system, e.g. the diffusion matrix will depend upon $\mu$. However, this does not alter our arguments below, and we therefore assume, for simplicity, that the asymptotic states are independent of $\mu$.

Consider next the parameter-dependent linearized operators defined by

$$
\mathcal{L}_{*}(\mu) u=-\partial_{y y} u-D^{-1}\left(c_{*}(\mu) \partial_{y} u+f^{\prime}\left(q_{*}(\cdot ; \mu) ; \mu\right) u\right),
$$

and

$$
\mathcal{M}_{k}(\mu) u=D\left(\partial_{y y}-k^{2}\right) u+c_{*}(\mu) \partial_{y} u+f^{\prime}\left(q_{*}(\cdot ; \mu) ; \mu\right) u .
$$

(For functions of several variables, we use' to denote the derivative with respect to the first variable.) We replace the hypothesis on stability by the following hypothesis requiring zero-stability (resp. transverse asymptotic stability) for $\mu<0$, and instability (resp. transverse instability) for $\mu>0$.

Hypothesis 4.6 (Onset of transverse instability). Assume that the travelling waves are transversely asymptotically stable if $\mu<0$ and transversely unstable if $\mu>0$. More precisely, assume that the essential spectrum of $\mathcal{M}_{0}$ is strictly contained in the left half plane and zero is the only eigenvalue in the closed right half plane, with algebraic multiplicity one, and that the spectra of $\mathcal{M}_{k}(0)$, for $k \neq 0$ are strictly contained in the left half plane. We assume that the unique eigenvalue $\lambda_{\mathrm{d}}(k ; \mu)$ of $\mathcal{M}_{k}(\mu), k \sim 0$, with $\lambda_{\mathrm{d}}(0 ; \mu)=\lambda_{\mathrm{d}}^{\prime}(0 ; \mu)=0$ satisfies $\lambda_{\mathrm{d}}^{\prime \prime}(0 ; 0)=0$, $\partial_{\mu} \lambda_{d}^{\prime \prime}(0 ; 0)>0$, and $\lambda_{d}^{(4)}(0 ; 0)<0$; see Fig. 6 .

Hypothesis 4.6 implies that the operators $\mathcal{M}_{k}(\mu)$, defined in (2.8), satisfy Hypothesis 2.3 if $\mu<0$ and Hypothesis 4.2 if $\mu>0$.

Theorem 4. Under the above Hypotheses, there exists $\mu_{*}>0$ such that for all $\mu \in\left(0, \mu_{*}\right)$, the system (4.10) possesses a symmetric exterior corner defect with speed $c_{\mathrm{e}}(\mu)=c_{*}+\mathrm{O}\left(\mu^{3}\right)$ and asymptotic angles $\vartheta_{ \pm}(\mu)=$ $\mathrm{O}\left(\mu^{3 / 2}\right)$.

This theorem will be a consequence of the analysis below. In addition, we will see that the exterior corner is accompanied by a plethora of other defects, indeed a countable family of exterior corners, interior corners, and steps. Most of them can be viewed as the spatial (in $x$ ) juxtaposition of two "elementary" defects, an exterior and an interior corner.

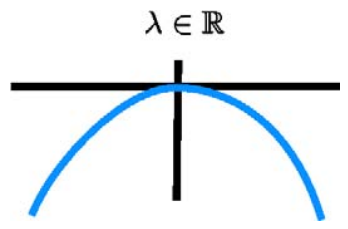

(a)

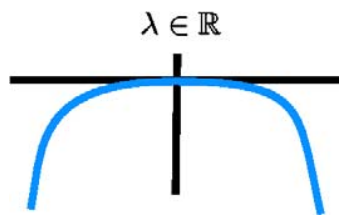

(b)

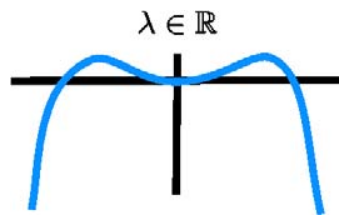

(c)

Fig. 6. The critical spectra of $\mathcal{M}_{k}(\mu)$ parameterized by $k$, before (a), at (b), and beyond threshold (c). 
As in the proof of Theorem 1, we rewrite the travelling-wave equation (4.10) as a first-order system in $x$ on the Hilbert space $Y=\left(H^{1} \times L^{2}\right)\left(\mathbb{R}, \mathbb{R}^{N}\right)$,

$$
\boldsymbol{u}_{x}=\mathcal{A}(c) \boldsymbol{u}+\mathcal{F}(\boldsymbol{u} ; \mu),
$$

where $\boldsymbol{u}=(u, v)^{T}$,

$$
\mathcal{A}(c)=\left(\begin{array}{cc}
0 & \mathrm{id} \\
-\partial_{y y}-D^{-1} c \partial_{y} & 0
\end{array}\right), \quad \mathcal{F}(\boldsymbol{u} ; \mu)=\left(\begin{array}{c}
0 \\
-D^{-1} f(u ; \mu)
\end{array}\right) .
$$

Hypothesis 4.5 on minimal spectrum in the origin shows that (4.12) possesses a smooth family of equilibria

$$
\boldsymbol{q}_{*}^{\xi}(\mu)=\left(\begin{array}{c}
q_{*}^{\xi}(\cdot ; \mu) \\
0
\end{array}\right)=\left(\begin{array}{c}
q_{*}(\cdot+\xi ; \mu) \\
0
\end{array}\right) .
$$

The linearization of (4.12) about $\boldsymbol{q}_{*}^{0}(\mu)$ is given by the operator

$$
\mathcal{A}_{*}(\mu)=\left(\begin{array}{cc}
0 & \mathrm{id} \\
\mathcal{L}_{*}(\mu) & 0
\end{array}\right)
$$

with $\mathcal{L}_{*}(\mu)$ the linear operator defined in (4.11). From Hypothesis 4.5 we conclude that

$$
\operatorname{spec} \mathcal{A}_{*}(0) \cap\{|\operatorname{Re} \lambda| \leqslant \epsilon\}=\{0\},
$$

for some $\epsilon>0$, and that zero is an eigenvalue with algebraic multiplicity four and geometric multiplicity one.

The kernel and generalized kernel of $\mathcal{A}_{*}(0)$ are spanned by

$$
\operatorname{ker} \mathcal{A}_{*}(0)=\operatorname{span}\left(\boldsymbol{e}_{0}\right), \quad \boldsymbol{e}_{0}=\left(\begin{array}{c}
q_{*}^{\prime}(\cdot ; 0) \\
0
\end{array}\right), \quad \operatorname{gker} \mathcal{A}_{*}(0)=\operatorname{span}\left(\boldsymbol{e}_{0}, \boldsymbol{e}_{1}, \boldsymbol{e}_{2}, \boldsymbol{e}_{3}\right),
$$

where

$$
\boldsymbol{e}_{1}=\left(\begin{array}{c}
0 \\
q_{*}^{\prime}(\cdot ; 0)
\end{array}\right), \quad \boldsymbol{e}_{2}=\left(\begin{array}{c}
r_{*}(\cdot) \\
0
\end{array}\right), \quad \boldsymbol{e}_{3}=\left(\begin{array}{c}
0 \\
r_{*}(\cdot)
\end{array}\right),
$$

are such that $\mathcal{A}_{*}(0) \boldsymbol{e}_{j+1}=\boldsymbol{e}_{j}$. Here $r_{*}$ is the principal vector to the zero eigenvalue of $\mathcal{L}_{*}(0), \mathcal{L}_{*}(0) r_{*}=q_{*}^{\prime}(\cdot ; 0)$. The kernel and generalized kernel of the adjoint $\mathcal{A}_{*}^{\text {ad }}(0)$ are given by

$$
\operatorname{ker} \mathcal{A}_{*}^{\mathrm{ad}}(0)=\operatorname{span}\left(\boldsymbol{e}_{0}^{\mathrm{ad}}\right), \quad \boldsymbol{e}_{0}^{\mathrm{ad}}=\left(\begin{array}{c}
0 \\
q_{*}^{\mathrm{ad}}(\cdot)
\end{array}\right), \quad \operatorname{gker} \mathcal{A}_{*}^{\mathrm{ad}}(0)=\operatorname{span}\left(\boldsymbol{e}_{0}^{\mathrm{ad}}, \boldsymbol{e}_{1}^{\mathrm{ad}}, \boldsymbol{e}_{2}^{\mathrm{ad}}, \boldsymbol{e}_{3}^{\mathrm{ad}}\right)
$$

where

$$
\boldsymbol{e}_{1}^{\mathrm{ad}}=\left(\begin{array}{c}
q_{*}^{\mathrm{ad}}(\cdot) \\
0
\end{array}\right), \quad \boldsymbol{e}_{2}^{\mathrm{ad}}=\left(\begin{array}{c}
0 \\
r_{*}^{\mathrm{ad}}(\cdot)
\end{array}\right), \quad \boldsymbol{e}_{3}^{\mathrm{ad}}=\left(\begin{array}{c}
r_{*}^{\mathrm{ad}}(\cdot) \\
0
\end{array}\right),
$$

are such that $\mathcal{A}_{*}^{\mathrm{ad}}(0) \boldsymbol{e}_{j+1}^{\mathrm{ad}}=\boldsymbol{e}_{j}^{\mathrm{ad}}$ and $\left(\boldsymbol{e}_{j}^{\mathrm{ad}}, \boldsymbol{e}_{3-i}\right)_{L^{2} \times L^{2}}=\delta_{i j}$. In particular, we have $\mathcal{L}_{*}^{\mathrm{ad}}(0) q_{*}^{\mathrm{ad}}=0$ and $\mathcal{L}_{*}^{\mathrm{ad}}(0) r_{*}^{\mathrm{ad}}=$ $q_{*}^{\text {ad }}$. The projection $P: Y \rightarrow Y$ on the generalized kernel is then given through

$$
P \boldsymbol{u}=\left\langle\boldsymbol{e}_{3}^{\mathrm{ad}}, \boldsymbol{u}\right\rangle_{L^{2} \times L^{2}} \boldsymbol{e}_{0}+\left\langle\boldsymbol{e}_{2}^{\mathrm{ad}}, \boldsymbol{u}\right\rangle_{L^{2} \times L^{2}} \boldsymbol{e}_{1}+\left\langle\boldsymbol{e}_{1}^{\mathrm{ad}}, \boldsymbol{u}\right\rangle_{L^{2} \times L^{2}} \boldsymbol{e}_{2}+\left\langle\boldsymbol{e}_{0}^{\mathrm{ad}}, \boldsymbol{u}\right\rangle_{L^{2} \times L^{2}} \boldsymbol{e}_{3} .
$$

Similarly, to the shifted equilibria $\boldsymbol{q}_{*}^{\xi}(0)$ we introduce the shifted linear operator $\mathcal{A}_{*}^{\xi}(0)$, the shifted eigenvector $\boldsymbol{e}_{0}^{\xi}=\left(\left(q_{*}^{\prime}\right)^{\xi}(\cdot ; 0), 0\right)^{T}$, and analogously $\boldsymbol{e}_{j}^{\xi}, \boldsymbol{e}_{j}^{\mathrm{ad}, \xi}, P^{\xi}, \mathcal{A}_{*}^{\text {ad, } \xi}(0)$

We seek solutions of (4.12) of the form

$$
\boldsymbol{u}=\boldsymbol{q}_{*}^{\xi}(\mu)+\eta_{1} \boldsymbol{e}_{1}^{\xi}+\eta_{2} \boldsymbol{e}_{2}^{\xi}+\eta_{3} \boldsymbol{e}_{3}^{\xi}+\boldsymbol{w}^{\xi}, \quad \text { with } P^{\xi} \boldsymbol{w}^{\xi}=P \boldsymbol{w}=0
$$

Following the proof of Theorem 1 - substituting (4.15) into (4.12), then taking successively the scalar product with $\boldsymbol{e}_{j}^{\mathrm{ad}, \xi}$, projecting with id $-P^{\xi}$, and finally applying the center manifold reduction - we find the following reduced system for $\eta_{1}, \eta_{2}$, and $\eta_{3}$, 


$$
\begin{aligned}
\eta_{1 x}= & \eta_{2}-\left\langle r_{*}^{\mathrm{ad}}, D^{-1} q_{*}^{\prime}(0)\right| \tilde{c}+\mathrm{O}\left(|\tilde{c}|\left(|\mu|+\left|\eta_{2}\right|\right)+|\mu|\left|\eta_{2}\right|+\left|\eta_{1}\right|\left(\left|\eta_{1}\right|+\left|\eta_{3}\right|\right)+\left|\eta_{2}\right|^{2}\right), \\
\eta_{2 x}= & \eta_{3}-\gamma_{01} \mu \eta_{1}+\mathrm{O}\left(\left|\eta_{1}\right|\left|\eta_{2}\right|+|\mu|\left|\eta_{1}\right|(|\tilde{c}|+|\mu|)+\left|\eta_{1}\right|^{2}\left(\left|\eta_{1}\right|+\left|\eta_{3}\right|\right)\right), \\
\eta_{3 x}= & -\left\langle q_{*}^{\mathrm{ad}}, D^{-1} q_{*}^{\prime}(0)\right| \tilde{c}-\left\langle q_{*}^{\mathrm{ad}}, q_{*}^{\prime \prime}(0)\right| \eta_{1}^{2}-\gamma_{02} \mu \eta_{2}+\mathrm{O}\left(|\tilde{c}|\left(|\mu|+\left|\eta_{2}\right|\right)+\left|\eta_{1}\right|\left|\eta_{3}\right|\right. \\
& \left.+\left|\eta_{2}\right|\left(|\mu|^{2}+\left|\eta_{2}\right|\right)+\left|\eta_{1}\right|^{2}\left(|\tilde{c}|+|\mu|+\left|\eta_{2}\right|+\left|\eta_{1}\right|^{2}\right)\right)
\end{aligned}
$$

in which $\tilde{c}=c-c_{*}(\mu), q_{*}(0)=q_{*}(\cdot, 0)$, and

$$
\begin{aligned}
& \gamma_{01}=\left\langle q_{*}^{\mathrm{ad}}, \partial_{\mu} q_{*}^{\prime}(0)\right\rangle, \\
& \gamma_{02}=\left\langle q_{*}^{\mathrm{ad}}, c_{*}^{\prime}(0) D^{-1} r_{*}^{\prime}+D^{-1}\left(D_{u u} f\left(q_{*}(0) ; 0\right) \partial_{\mu} q_{*}(0)+D_{\mu u} f\left(q_{*}(0) ; 0\right) r_{*}\right)\right\rangle .
\end{aligned}
$$

The equation for $\xi$ decouples,

$$
\xi_{x}=\eta_{1}+\mathrm{O}\left(\left|\eta_{1}\right|\left(|\mu|+\left|\eta_{2}\right|+\left|\eta_{1}\right|^{2}+\left|\eta_{1}\right|\left|\eta_{3}\right|\right)\right) .
$$

We now introduce the following (Kuramoto-Sivashinsky) scaling

$$
x=|\mu|^{-1 / 2} \zeta, \quad \eta_{1}=|\mu|^{3 / 2} \bar{\eta}_{1}, \quad \eta_{2}=|\mu|^{2} \bar{\eta}_{2}, \quad \eta_{3}=|\mu|^{5 / 2} \bar{\eta}_{3}, \quad \tilde{c}=|\mu|^{3} \bar{c} .
$$

Then the reduced system (4.16)-(4.18) becomes

$$
\begin{aligned}
& \bar{\eta}_{1, \zeta}=\bar{\eta}_{2}+\mathrm{O}(|\mu|), \\
& \bar{\eta}_{2, \zeta}=\bar{\eta}_{3}-\gamma_{01} \operatorname{sign}(\mu) \bar{\eta}_{1}+\mathrm{O}(|\mu|), \\
& \bar{\eta}_{3, \zeta}=-\left\langle q_{*}^{\mathrm{ad}}, D^{-1} q_{*}^{\prime}(0)\right\rangle \bar{c}-\left\langle q_{*}^{\mathrm{ad}}, q_{*}^{\prime \prime}(0)\right| \bar{\eta}_{1}^{2}-\gamma_{02} \operatorname{sign}(\mu) \bar{\eta}_{2}+\mathrm{O}(|\mu|) .
\end{aligned}
$$

At $\mu=0$ we find

$$
\bar{\eta}_{1, \zeta \zeta \zeta}=-\left\langle q_{*}^{\mathrm{ad}}, D^{-1} q_{*}^{\prime}(0)\right\rangle \bar{c}-\left(\gamma_{01}+\gamma_{02}\right) \operatorname{sign}(\mu) \bar{\eta}_{1, \zeta}-\left\langle q_{*}^{\mathrm{ad}}, q_{*}^{\prime \prime}(0)\right) \bar{\eta}_{1}^{2} .
$$

Taking the derivative with respect to $\zeta$ gives the steady-state Kuramoto-Sivashinsky equation.

The coefficients appearing in (4.23) are computed from the eigenvalue problem for $\mathcal{M}_{k}(\mu)$,

$$
D\left(\partial_{y y}-k^{2}\right) u(k ; \mu)+c_{*}(\mu) \partial_{y} u(k ; \mu)+f^{\prime}\left(q_{*}(\cdot ; \mu) ; \mu\right) u(k ; \mu)=\lambda_{\mathrm{d}}(k ; \mu) u(k ; \mu),
$$

where $u(k ; \mu)$ represents the normalized eigenvector to the eigenvalue $\lambda_{\mathrm{d}}(k ; \mu)$. First, by taking the second derivative of (4.24) with respect to $k$ at $k=0$ and $\mu=0$ we find

$$
D \partial_{y y} u^{\prime \prime}(0 ; 0)+c_{*}(0) \partial_{y} u^{\prime \prime}(0 ; 0)+f^{\prime}\left(q_{*}(\cdot ; 0) ; 0\right) u^{\prime \prime}(0 ; 0)=2 D q_{*}^{\prime}(\cdot ; 0),
$$

since $\lambda_{\mathrm{d}}^{\prime \prime}(0 ; 0)=0$. Then $\mathcal{L}_{*}(0) u^{\prime \prime}(0 ; 0)=-2 q_{*}^{\prime}(\cdot ; 0)$, so that

$$
r_{*}=-\frac{1}{2} u^{\prime \prime}(0 ; 0) \text {. }
$$

Then, by using successively the derivatives $\partial_{k}^{4}$ and $\partial_{k}^{2} \partial_{\mu}$ of (4.24) at $k=0$ and $\mu=0$ we obtain

$$
\left\langle q_{*}^{\text {ad }}, D^{-1} q_{*}^{\prime}(\cdot ; 0)\right\rangle=\frac{24}{\lambda_{\mathrm{d}}^{(4)}(0 ; 0)}<0, \quad \gamma_{01}+\gamma_{02}=-\frac{12 \partial_{\mu} \lambda_{\mathrm{d}}^{\prime \prime}(0 ; 0)}{\lambda_{\mathrm{d}}^{(4)}(0 ; 0)}>0 .
$$

Finally, we have

$$
\left\langle q_{*}^{\mathrm{ad}}, q_{*}^{\prime \prime}\right\rangle=-\frac{c_{*}(0)}{2}\left\langle q_{*}^{\mathrm{ad}}, D^{-1} q_{*}^{\prime}(\cdot ; 0)\right\rangle=-\frac{12 c_{*}(0)}{\lambda_{\mathrm{d}}^{(4)}(0 ; 0)}>0,
$$

and (4.23) becomes

$$
\bar{\eta}_{1, \zeta \zeta \zeta}=-\frac{12}{\lambda_{\mathrm{d}}^{(4)}(0 ; 0)}\left(2 \bar{c}-\partial_{\mu} \lambda_{\mathrm{d}}^{\prime \prime}(0 ; 0) \operatorname{sign}(\mu) \bar{\eta}_{1, \zeta}-c_{*}(0) \bar{\eta}_{1}^{2}\right) .
$$


Solutions of the Kuramoto-Sivashinsky equation (4.25) have been described in [38]. The equation is written as dynamical system

$$
\begin{aligned}
& u_{1, \zeta}=u_{2}, \\
& u_{2, \zeta}=u_{3}, \\
& u_{3, \zeta}=-\frac{12}{\lambda_{\mathrm{d}}^{(4)}(0 ; 0)}\left(2 \bar{c}-\partial_{\mu} \lambda_{\mathrm{d}}^{\prime \prime}(0 ; 0) \operatorname{sign}(\mu) u_{2}-c_{*}(0) u_{1}^{2}\right),
\end{aligned}
$$

in which $u_{1}=\bar{\eta}_{1}$. This system has two fixed points

$$
P_{ \pm}=\left( \pm \sqrt{2 \bar{c} / c_{*}(0)}, 0,0\right)
$$

if $\bar{c}>0$ (recall that $c_{*}(0)>0$ ), and no fixed points if $\bar{c}<0$. Again these fixed points correspond to a rotated planar travelling wave solution of (4.10). At $\bar{c}=0$, there is one fixed point at the origin. The eigenvalues of the linearization about the origin are

$$
\nu_{0}=0, \quad v_{1,2}= \pm \sqrt{12 \partial_{\mu} \lambda_{d}^{\prime \prime}(0 ; 0) \operatorname{sign}(\mu) / \lambda_{d}^{(4)}(0 ; 0)},
$$

so that the nontrivial eigenvalues $\nu_{1,2}$ are both real if $\mu<0$, and purely imaginary if $\mu>0$.

We assume now that $\bar{c}>0$. Then the eigenvalues $v_{j}^{ \pm}$of the linearization about $P_{ \pm}$satisfy

$$
\left(v_{j}^{ \pm}\right)^{3}-\frac{12 \partial_{\mu} \lambda_{\mathrm{d}}^{\prime \prime}(0 ; 0)}{\lambda_{\mathrm{d}}^{(4)}(0 ; 0)} \operatorname{sign}(\mu) v_{j}^{ \pm} \mp \frac{24 \sqrt{2 \bar{c}}}{\sqrt{c_{*}(0)} \lambda_{\mathrm{d}}^{(4)}(0 ; 0)}=0 .
$$

It is straightforward to check that at $P_{-}$(resp. $P_{+}$) there is one positive (resp. negative) eigenvalue, and a pair of eigenvalues with negative (resp. positive) real part. These eigenvalues are real for $\mu<0$ and bounded values of $\bar{c}$, $\bar{c} \in\left(0, \bar{c}_{*}\right)$, for some $\bar{c}_{*}>0$, and complex conjugated otherwise. Therefore, at $P_{-}$(resp. $\left.P_{+}\right)$we have a 1D-unstable (resp. 1D-stable) manifold, and a 2D-stable (resp. 2D-unstable) manifold. Heteroclinic and homoclinic connections of (4.26) are found as intersections of these manifolds.

A summary of results on existence of heteroclinics and homoclinics in the case $\mu>0$ can be found in [38]. The 2D manifolds of the fixed points intersect transversely along a nontrivial trajectory, at least for large values of $\bar{c}$. The trajectory represents a symmetric heteroclinic connection between $P_{+}$, as $\zeta \rightarrow-\infty$, and $P_{-}$, as $\zeta \rightarrow \infty$, which will persist for the reduced system (4.20)-(4.22). It corresponds to an interior corner to (4.10).

The 1D manifolds of the fixed points may intersect for special values of $\bar{c}$ and form a 1D-1D heteroclinic connection between $P_{+}$, as $\zeta \rightarrow \infty$, and $P_{-}$, as $\zeta \rightarrow-\infty$. There is a countable collection of such orbits. The simplest takes the explicit form

$$
u_{1}(\zeta)=\alpha\left(-9 \tanh (\beta \zeta)+11 \tanh ^{3}(\beta \zeta)\right)
$$

for some suitably chosen constants $\alpha$ and $\beta$, and speed $\bar{c}$; see [38] and Appendix A. Such heteroclinic connections correspond to exterior corners in the reaction-diffusion system. Some of the more complicated exterior corners can be viewed as a zig-zag type, widely spaced conjunction of copies of this simplest exterior corner and interior corners. In Appendix A, we provide a Melnikov-type computation, that shows robustness of this exterior corner with respect to perturbations, induced by the higher order terms in the Taylor expansion on the center-manifold. In particular, this will prove Theorem 4.

In addition to these heteroclinic connections, the system (4.26) possesses homoclinic connections to both fixed points $P_{-}$and $P_{+}$, found as intersections of the 1D and 2D manifolds. These homoclinic connections correspond to steps in the reaction-diffusion system. They can be viewed as the widely-spaced superposition of an interior and an exterior corner of equal height. In fact, for speeds close to the speed of an interior corner, homoclinic orbits can be found in a heteroclinic loop bifurcation between the equilibria $P_{-}$and $P_{+}$[5]. For a robust unfolding, two parameters, given by the speed $c$ in the $y$-direction and the speed $c_{x}$ along the interface are needed; see Remark 2.9, where the drift term $c_{x} \partial_{x} u$ is discussed. 
Remark 4.7 (Stability). Few analytical results seem to be known on stability. However, in our case, since the flat surface is unstable, the spectrum of the linearization at any of the corner defects contains unstable essential spectrum in the right half plane. A more precise analysis of the Kuramoto-Sivashinsky equation shows that the exterior corners are in fact absolutely unstable: the Green's function of the linearization at the asymptotic states possesses a branch point in the unstable right half plane such that there do not exist exponential weights that would push the essential spectrum in the left stable complex half-plane; see [4,50] for background on convective and absolute instabilities.

\section{Corners in oscillatory wave-propagation}

In this section we look for corner defects in pulsating front propagation. Throughout this section, the planar wave is assumed to be a modulated wave solution $u(x, y, t)=q_{*}(y, \omega t)$ of the reaction-diffusion system (2.1) connecting two homogeneous equilibria $q_{ \pm}$as $y \rightarrow \pm \infty$, and with $q_{*}$ being $2 \pi$-periodic in its second argument. The profile $q_{*}(\cdot, \cdot)$ satisfies

$$
D \partial_{y y} u+c \partial_{y} u+f(u)-\omega \partial_{t} u=0,
$$

for some speed $c=c_{*}$ and frequency $\omega=\omega_{*}$, where $\omega t$ is replaced by $t$. Corner defects are found as bounded solutions to

$$
D \Delta_{x, y} u+c \partial_{y} u+f(u)-\omega \partial_{t} u=0,
$$

which are $2 \pi$-periodic in $t$.

The main hypotheses and the reduction procedure are described in Section 5.1. At lowest order, the reduced system is a quadratic differential system in the plane. For $c=c_{*}$ and $\omega=\omega_{*}$, the origin is typically an isolated equilibrium, and, under certain algebraic conditions on the different coefficients, orbits which are homoclinic to the origin exist; see Section 5.2. These orbits decay algebraically as $|x| \rightarrow \infty$ and correspond to holes in the reactiondiffusion system. For slightly different speeds, $c>c_{*}$, interior corners exist, just as in the case of travelling fronts discussed in Section 2.

\subsection{Hypotheses and reduction}

We will assume existence of a planar modulated wave connecting two homogeneous equilibria.

Hypothesis 5.1 (Existence). We assume that there exist positive constants $c_{*}, \omega_{*}$, and homogeneous states $q_{ \pm}$such that there exists an $x$-independent planar modulated-wave solution $q_{*}(y, t)$ of (5.2) which is $2 \pi$-periodic in $t$, with $\partial_{t} q_{*} \not \equiv 0$, and which connects $q_{-}$and $q_{+}$, that is,

$$
q_{*}(y, t) \rightarrow q_{+} \text {for } y \rightarrow+\infty, \quad q_{*}(y, t) \rightarrow q_{-} \text {for } y \rightarrow-\infty,
$$

uniformly in $t$.

The second assumption is again concerned with stability of the above modulated wave. Therefore, consider the closed unbounded linearized operator defined by

$$
\mathcal{L}_{*} u=-\partial_{y y} u-D^{-1}\left(c_{*} \partial_{y} u+f^{\prime}\left(q_{*}\right) u-\omega_{*} \partial_{t} u\right),
$$

on the Hilbert space $L^{2}\left(\mathbb{R} \times S^{1}, \mathbb{R}^{N}\right)$ of functions which are $2 \pi$-periodic in $t$. Notice that $\partial_{y} q_{*}$ and $\partial_{t} q_{*}$ always belong to the kernel of $\mathcal{L}_{*}$ due to translation invariances in $y$ and $t$.

Hypothesis 5.2 (Zero-stability). We assume that $\mathcal{L}_{*}-\lambda$ id is invertible for all $\lambda<0$ and that $\lambda=0$ is an isolated eigenvalue with algebraic and geometric multiplicity two. 
As in the case of travelling waves this hypothesis is related to stability properties of the modulated wave. Consider the linearized operator

$$
\mathcal{M}_{*} u=D \Delta_{x, y} u+c_{*} \partial_{y} u+f^{\prime}\left(q_{*}\right) u-\omega_{*} \partial_{t} u,
$$

and its Fourier conjugates

$$
\mathcal{M}_{k} u=D\left(\partial_{y y}-k^{2}\right) u+c_{*} \partial_{y} u+f^{\prime}\left(q_{*}\right) u-\omega_{*} \partial_{t} u,
$$

on the Hilbert spaces $L^{2}\left(\mathbb{R}^{2} \times S^{1}, \mathbb{R}^{N}\right)$ and $L^{2}\left(\mathbb{R} \times S^{1}, \mathbb{R}^{N}\right)$, respectively. An argument similar to [51, Theorem 2.7] shows that the union of the spectra of $\mathcal{M}_{k}$ gives the Floquet spectrum of the linearized period map at the modulated wave, that is, $\lambda$ is the spectrum of $\mathcal{M}_{k}$ for some $k$ if and only if $\mathrm{e}^{2 \pi \lambda / \omega_{*}}$ is in the spectrum of the time- $2 \pi / \omega_{*}$ map of the linearized equation

$$
\partial_{t} u=D\left(\partial_{y y} u+\partial_{x x} u\right)+c_{*} \partial_{y} u+f^{\prime}\left(q_{*}\right) u .
$$

Hypothesis 5.2 is a consequence of the following, slightly stronger hypothesis on transverse stability of the modulated front.

Hypothesis 5.3 (Transverse asymptotic stability). Assume that the spectrum of $\mathcal{M}_{0}$ is contained in the closed left half plane and zero is an isolated eigenvalue with algebraic and geometric multiplicity two, and that the spectra of $\mathcal{M}_{k}$, for $k \neq 0$ are strictly contained in the left half plane. Furthermore, assume that the $2 \times 2$ matrix $\Lambda_{\mathrm{d}}(k)$ with $\Lambda_{\mathrm{d}}(0)=\Lambda_{\mathrm{d}}^{\prime}(0)=0$, representing the smooth continuation for $k \sim 0$ of the action of $\mathcal{M}_{0}$ on its kernel, satisfies $\operatorname{Re} \operatorname{spec} \Lambda_{\mathrm{d}}^{\prime \prime}(0)<0$.

In order to see that Hypothesis 5.3 implies Hypothesis 5.2, we first note that a kernel of $\mathcal{L}_{*}-\lambda$ id for $\lambda<0$ would induce a kernel of $\mathcal{M}_{k}$ for $\lambda=-k^{2}$. By a similar argument, the geometric multiplicity of $\lambda=0$ as an eigenvalue of $\mathcal{L}_{*}$ is two. To conclude, we observe that generalized eigenvectors can be found by solving $\left.\partial_{k}^{j}\right|_{k=0} \mathcal{M}_{k} u=0$. In particular, algebraic multiplicity higher than two is equivalent to a kernel of $\Lambda_{\mathrm{d}}^{\prime \prime}(0)$; see also [57, Remark 2.12, Lemma 2.30].

Theorem 5. There exists an open class of reaction-diffusion systems which satisfy Hypotheses 5.2 and 5.3, that possess a one-parameter family of holes, close to a stable planar modulated front having the same speed and frequency.

The theorem will be a consequence of the following discussion of the general reduction procedure and of the analysis of the reduced equations in Section 5.2.

We now describe the reduction procedure and derive a reduced system which describes all bounded solutions to (5.2) close to the planar modulated wave $q_{*}$.

We set $\boldsymbol{u}=(u, v)^{T}$ and rewrite Eq. (5.2) as a dynamical system

$$
\boldsymbol{u}_{x}=\mathcal{A}(c, \omega) \boldsymbol{u}+\mathcal{F}(\boldsymbol{u}),
$$

on the Hilbert space $Y=\left(H^{1,1 / 2} \times L^{2}\right)\left(\mathbb{R} \times S^{1}, \mathbb{R}^{N}\right)$, where $H^{1,1 / 2}$ denotes the fractional derivative anisotropic Sobolev space defined via interpolation from the integer anisotropic spaces

$$
H^{k, l}\left(\mathbb{R} \times S^{1}, \mathbb{R}^{N}\right)=\left\{u \in L^{2}\left(\mathbb{R} \times S^{1}, \mathbb{R}^{N}\right) ; \partial_{y}^{(i)} \partial_{t}^{(j)} u \in L^{2}\left(\mathbb{R} \times S^{1}, \mathbb{R}^{N}\right), 0 \leqslant i \leqslant k, 0 \leqslant j \leqslant l\right\},
$$

with $k, l \in \mathbb{N}$. The linear and nonlinear part of (5.6) are given by

$$
\mathcal{A}(c, \omega)=\left(\begin{array}{cc}
0 & \mathrm{id} \\
-\partial_{y y}-D^{-1} c \partial_{y}+D^{-1} \omega \partial_{t} & 0
\end{array}\right), \quad \mathcal{F}(\boldsymbol{u})=\left(\begin{array}{c}
0 \\
-D^{-1} f(u)
\end{array}\right) .
$$


This equation possesses two continuous translation symmetries, induced by the $y$ - and the $t$-shifts, $\xi: \boldsymbol{u}(\cdot, \cdot) \mapsto$ $\boldsymbol{u}(\cdot+\xi, \cdot)$ and $\tau: \boldsymbol{u}(\cdot, \cdot) \mapsto \boldsymbol{u}(\cdot, \cdot+\tau)$, respectively. It also has a reversibility symmetry $(u, v)^{T} \mapsto R(u, v)^{T}:=$ $(u,-v)^{T}, x \mapsto-x$.

Hypothesis 5.1 shows that (5.6) has a two-parameter family of equilibria

$$
\boldsymbol{q}_{*}^{\xi, \tau}=\left(\begin{array}{c}
q_{*}^{\xi, \tau}(\cdot, \cdot) \\
0
\end{array}\right)=\left(\begin{array}{c}
q_{*}(\cdot+\xi, \cdot+\tau) \\
0
\end{array}\right) .
$$

The linearization of (5.6) about $\boldsymbol{q}_{*}^{0,0}$ is given by the operator

$$
\mathcal{A}_{*}=\left(\begin{array}{cc}
0 & \text { id } \\
\mathcal{L}_{*} & 0
\end{array}\right)
$$

with $\mathcal{L}_{*}$ the linear operator defined in (5.3). The domain of definition of $\mathcal{A}_{*}$ is easily seen to be $Y^{1}:=\left(H^{2,1} \times\right.$ $\left.H^{1,1 / 2}\right)\left(\mathbb{R} \times S^{1}, \mathbb{R}^{N}\right)$. Hypothesis 5.2 implies that the spectrum of $\mathcal{A}_{*}$ satisfies

$$
\operatorname{spec} \mathcal{A}_{*} \cap\{|\operatorname{Re} \lambda| \leqslant \epsilon\}=\{0\},
$$

in which the origin is an eigenvalue with geometric multiplicity two and algebraic multiplicity four.

The kernel of $\mathcal{A}_{*}$ is spanned by the partial derivatives of $\boldsymbol{q}_{*}^{0,0}$ with respect to $y$ and $t$,

$$
\operatorname{ker} \mathcal{A}_{*}=\operatorname{span}\left(\boldsymbol{e}_{0 y}, \boldsymbol{e}_{0 t}\right), \quad \boldsymbol{e}_{0 y}=\left(\begin{array}{c}
\partial_{y} q_{*} \\
0
\end{array}\right), \quad \boldsymbol{e}_{0 t}=\left(\begin{array}{c}
\partial_{t} q_{*} \\
0
\end{array}\right),
$$

and the four-dimensional generalized kernel is spanned by

$$
\operatorname{gker} \mathcal{A}_{*}=\operatorname{span}\left(\boldsymbol{e}_{0 y}, \boldsymbol{e}_{0 t}, \boldsymbol{e}_{1 y}, \boldsymbol{e}_{1 t}\right), \quad \boldsymbol{e}_{1 y}=\left(\begin{array}{c}
0 \\
\partial_{y} q_{*}
\end{array}\right), \quad \boldsymbol{e}_{1 t}=\left(\begin{array}{c}
0 \\
\partial_{t} q_{*}
\end{array}\right) .
$$

We construct a dual basis

$$
\boldsymbol{e}_{0 y}^{\mathrm{ad}}=\left(\begin{array}{c}
0 \\
q_{* y}^{\mathrm{ad}}
\end{array}\right), \quad \boldsymbol{e}_{0 t}^{\mathrm{ad}}=\left(\begin{array}{c}
0 \\
q_{* t}^{\mathrm{ad}}
\end{array}\right), \quad \boldsymbol{e}_{1 y}^{\mathrm{ad}}=\left(\begin{array}{c}
q_{* y}^{\mathrm{ad}} \\
0
\end{array}\right), \quad \boldsymbol{e}_{1 t}^{\mathrm{ad}}=\left(\begin{array}{c}
q_{* t}^{\mathrm{ad}} \\
0
\end{array}\right),
$$

in which $q_{* y}^{\text {ad }}$ and $q_{* t}^{\text {ad }}$ span the kernel of the adjoint operator $\mathcal{L}_{*}^{\text {ad }}$, such that

$$
\left\langle\boldsymbol{e}_{j, y}^{\mathrm{ad}}, \boldsymbol{e}_{1-j, y}\right\rangle_{L^{2} \times L^{2}}=1, \quad\left\langle\boldsymbol{e}_{j, t}^{\mathrm{ad}}, \boldsymbol{e}_{1-j, t}\right\rangle_{L^{2} \times L^{2}}=1, \quad j=0,1,
$$

and all other scalar products vanish. The spectral projection $P: Y \rightarrow Y$ associated with the central eigenvalue 0 is given by

$$
P \boldsymbol{u}=\left\langle\boldsymbol{e}_{1 y}^{\mathrm{ad}}, \boldsymbol{u}\right\rangle_{L^{2} \times L^{2}} \boldsymbol{e}_{0 y}+\left\langle\boldsymbol{e}_{1 t}^{\mathrm{ad}}, \boldsymbol{u}\right\rangle_{L^{2} \times L^{2}} \boldsymbol{e}_{0 t}+\left\langle\boldsymbol{e}_{0 y}^{\mathrm{ad}}, \boldsymbol{u}\right\rangle_{L^{2} \times L^{2}} \boldsymbol{e}_{1 y}+\left\langle\boldsymbol{e}_{0 t}^{\mathrm{ad}}, \boldsymbol{u}\right\rangle_{L^{2} \times L^{2}} \boldsymbol{e}_{1 t} .
$$

Similarly, to the shifted equilibria $\boldsymbol{q}_{*}^{\xi, \tau}$ we introduce the shifted eigenvectors $\boldsymbol{e}_{j, y}^{\xi, \tau}, \boldsymbol{e}_{j, t}^{\xi, \tau}, \boldsymbol{e}_{j, y}^{\mathrm{ad}, \xi, \tau}, \boldsymbol{e}_{j, t}^{\mathrm{ad}, \xi, \tau}$, and the shifted projection $P^{\xi, \tau}$.

We set

$$
\boldsymbol{u}=\boldsymbol{q}_{*}^{\xi, \tau}+\eta \boldsymbol{e}_{1 y}^{\xi, \tau}+\rho \boldsymbol{e}_{1 t}^{\xi, \tau}+\boldsymbol{w}^{\xi, 0}, \quad \text { with } P^{\xi, \tau} \boldsymbol{w}^{\xi, 0}=P^{0, \tau} \boldsymbol{w}^{0,0}=0,
$$

where $\xi, \tau, \eta$, and $\rho$ are functions depending upon $x$. For technical reasons, we only shift the hyperbolic component $\boldsymbol{w}$ in the $y$-but not in the $t$-direction. A shift in $t$ would introduce a term $\tau_{x} \partial_{t} \boldsymbol{w}$ in the projected equation for $\boldsymbol{w}$. This nonlinear term would not be relatively bounded with respect to the linear part of the system and center-manifold theorems for this type of equations do not seem to be available.

Substituting (5.8) into (5.3) we find the system 


$$
\begin{aligned}
\xi_{x} \boldsymbol{e}_{0 y}^{\xi, \tau}+\tau_{x} \boldsymbol{e}_{0 t}^{\xi, \tau}+\eta_{x} \boldsymbol{e}_{1 y}^{\xi, \tau}+\rho_{x} \boldsymbol{e}_{1 t}^{\xi, \tau}+\eta \xi_{x} \partial_{y} \boldsymbol{e}_{1 y}^{\xi, \tau}+\eta \tau_{x} \partial_{t} \boldsymbol{e}_{1 y}^{\xi, \tau}+\rho \xi_{x} \partial_{y} \boldsymbol{e}_{1 t}^{\xi, \tau}+\rho \tau_{x} \partial_{t} \boldsymbol{e}_{1 t}^{\xi, \tau}+\partial_{x}\left(\boldsymbol{w}^{\xi, 0}\right) \\
=\mathcal{A}_{*}\left(\eta \boldsymbol{e}_{1 y}^{\xi, \tau}+\rho \boldsymbol{e}_{1 t}^{\xi, \tau}+\boldsymbol{w}^{\xi, 0}\right)+\mathcal{A}_{1}\left(c-c_{*}\right)\left(\boldsymbol{q}_{*}^{\xi, \tau}+\boldsymbol{w}^{\xi, 0}\right) \\
\quad+\mathcal{A}_{2}\left(\omega-\omega_{*}\right)\left(\boldsymbol{q}_{*}^{\xi, \tau}+\boldsymbol{w}^{\xi, 0}\right)+\mathcal{G}^{\xi, \tau}\left(\boldsymbol{w}^{\xi, 0}\right),
\end{aligned}
$$

in which $\mathcal{A}_{1}$ and $\mathcal{A}_{2}$ are obtained from

$$
\mathcal{A}(c, \omega)=\mathcal{A}\left(c_{*}, \omega_{*}\right)+\mathcal{A}_{1}\left(c-c_{*}\right)+\mathcal{A}_{2}\left(\omega-\omega_{*}\right),
$$

and

$$
\mathcal{G}^{\xi, \tau}\left(\boldsymbol{w}^{\xi, 0}\right)=\mathcal{F}\left(\boldsymbol{q}_{*}^{\xi, \tau}+\boldsymbol{w}^{\xi, 0}\right)-\mathcal{F}\left(\boldsymbol{q}_{*}^{\xi, \tau}\right)-D \mathcal{F}\left(\boldsymbol{q}_{*}^{\xi, \tau}\right) \boldsymbol{w}^{\xi, 0}
$$

Taking successively the scalar product of (5.9) with $\boldsymbol{e}_{1 y}^{\mathrm{ad}, \xi, \tau}, \boldsymbol{e}_{1 t}^{\mathrm{ad}, \xi, \tau}, \boldsymbol{e}_{0 y}^{\mathrm{ad}, \xi, \tau}, \boldsymbol{e}_{0 t}^{\mathrm{ad}, \xi, \tau}$, and projecting with id $-P^{\xi, \tau}$ we obtain the decomposed system

$$
\begin{aligned}
& \xi_{x}\left(1-\left\langle\partial_{y} \boldsymbol{e}_{1 y}^{\mathrm{ad}, 0, \tau}, \boldsymbol{w}^{0,0}\right\rangle\right)-\tau_{x}\left\langle\partial_{t} \boldsymbol{e}_{1 y}^{\mathrm{ad}, 0, \tau}, \boldsymbol{w}^{0,0}\right\rangle=\eta+\left\langle\boldsymbol{e}_{1 y}^{\mathrm{ad}, 0, \tau}, G^{0, \tau}\left(\boldsymbol{w}^{0,0}\right)\right\rangle, \\
& \tau_{x}\left(1-\left\langle\partial_{t} \boldsymbol{e}_{1 t}^{\mathrm{ad}, 0, \tau}, \boldsymbol{w}^{0,0}\right\rangle\right)-\xi_{x}\left\langle\partial_{x} \boldsymbol{e}_{1 t}^{\mathrm{ad}, 0, \tau}, \boldsymbol{w}^{0,0}\right\rangle=\rho+\left\langle\boldsymbol{e}_{1 t}^{\mathrm{ad}, 0, \tau}, G^{0, \tau}\left(\boldsymbol{w}^{0,0}\right)\right\rangle \text {, } \\
& \eta_{x}=-\eta \xi_{x}\left\langle\boldsymbol{e}_{0 y}^{\mathrm{ad}}, \partial_{y} \boldsymbol{e}_{1 y}\right\rangle-\eta \tau_{x}\left\langle\boldsymbol{e}_{0 y}^{\mathrm{ad}}, \partial_{t} \boldsymbol{e}_{1 y}\right\rangle-\rho \xi_{x}\left\langle\boldsymbol{e}_{0 y}^{\mathrm{ad}}, \partial_{y} \boldsymbol{e}_{1 t}\right\rangle-\rho \tau_{x}\left\langle\boldsymbol{e}_{0 y}^{\mathrm{ad}}, \partial_{t} \boldsymbol{e}_{1 t}\right\rangle \\
& +\xi_{x}\left\langle\partial_{y} \boldsymbol{e}_{0 y}^{\mathrm{ad}, 0, \tau}, \boldsymbol{w}^{0,0}\right\rangle+\tau_{x}\left\langle\partial_{t} \boldsymbol{e}_{0 y}^{\mathrm{ad}, 0, \tau}, \boldsymbol{w}^{0,0}\right\rangle+\left(c-c_{*}\right)\left\langle\boldsymbol{e}_{0 y}^{\mathrm{ad}, 0, \tau}, \mathcal{A}_{1}\left(\boldsymbol{q}_{*}^{0, \tau}+\boldsymbol{w}^{0,0}\right)\right\rangle \\
& +\left(\omega-\omega_{*}\right)\left\langle\boldsymbol{e}_{0 y}^{\mathrm{ad}, 0, \tau}, \mathcal{A}_{2}\left(\boldsymbol{q}_{*}^{0, \tau}+\boldsymbol{w}^{0,0}\right)\right\rangle+\left\langle\boldsymbol{e}_{0 y}^{\mathrm{ad}, 0, \tau}, G^{0, \tau}\left(\boldsymbol{w}^{0,0}\right)\right\rangle, \\
& \rho_{x}=-\eta \xi_{x}\left\langle\boldsymbol{e}_{0 t}^{\mathrm{ad}}, \partial_{y} \boldsymbol{e}_{1 y}\right\rangle-\eta \tau_{x}\left\langle\boldsymbol{e}_{0 t}^{\mathrm{ad}}, \partial_{t} \boldsymbol{e}_{1 y}\right\rangle-\rho \xi_{x}\left\langle\boldsymbol{e}_{0 t}^{\mathrm{ad}}, \partial_{y} \boldsymbol{e}_{1 t}\right\rangle-\rho \tau_{x}\left\langle\boldsymbol{e}_{0 t}^{\mathrm{ad}}, \partial_{t} \boldsymbol{e}_{1 t}\right\rangle \\
& +\xi_{x}\left\langle\partial_{y} e_{0 t}^{\mathrm{ad}, 0, \tau}, \boldsymbol{w}^{0,0}\right\rangle+\tau_{x}\left\langle\partial_{t} \boldsymbol{e}_{0 t}^{\mathrm{ad}, 0, \tau}, \boldsymbol{w}^{0,0}\right\rangle+\left(c-c_{*}\right)\left\langle\boldsymbol{e}_{0 t}^{\mathrm{ad}, 0, \tau}, \mathcal{A}_{1}\left(\boldsymbol{q}_{*}^{0, \tau}+\boldsymbol{w}^{0,0}\right)\right\rangle \\
& +\left(\omega-\omega_{*}\right)\left\langle\boldsymbol{e}_{0 t}^{\mathrm{ad}, 0, \tau}, \mathcal{A}_{2}\left(\boldsymbol{q}_{*}^{0, \tau}+\boldsymbol{w}^{0,0}\right)\right\rangle+\left\langle\boldsymbol{e}_{0 t}^{\mathrm{ad}, 0, \tau}, G^{0, \tau}\left(\boldsymbol{w}^{0,0}\right)\right\rangle, \\
& \boldsymbol{w}_{x}^{0,0}=\mathcal{A}_{*}^{0, \tau} \boldsymbol{w}^{0,0}-\xi_{x} \partial_{y} \boldsymbol{w}^{0,0}-\xi_{x}\left(\partial_{y} P^{0, \tau}\right) \boldsymbol{w}^{0,0} \\
& +\left(\mathrm{id}-P^{0, \tau}\right)\left(-\eta \xi_{x}\left(\partial_{y} \boldsymbol{e}_{1 y}^{0, \tau}\right)-\eta \tau_{x}\left(\partial_{t} \boldsymbol{e}_{1 y}^{0, \tau}\right)-\rho \xi_{x}\left(\partial_{y} \boldsymbol{e}_{1 t}^{0, \tau}\right)-\rho \tau_{x}\left(\partial_{t} \boldsymbol{e}_{1 t}^{0, \tau}\right)\right) \\
& +\left(\mathrm{id}-P^{0, \tau}\right)\left(\left(c-c_{*}\right) \mathcal{A}_{1}\left(\boldsymbol{q}_{*}^{0, \tau}+\boldsymbol{w}^{0,0}\right)+\left(\omega-\omega_{*}\right) \mathcal{A}_{2}\left(\boldsymbol{q}_{*}^{0, \tau}+\boldsymbol{w}^{0,0}\right)+G^{0, \tau}\left(\boldsymbol{w}^{0,0}\right)\right),
\end{aligned}
$$

in which we have used the invariance of the scalar products under the $y$-shift. Notice that we can invert the equations (5.10), (5.11) when $\boldsymbol{w}^{0,0}$ is small and obtain $\xi_{x}$ and $\tau_{x}$ in terms of $\eta, \rho$ and $\boldsymbol{w}^{0,0}$. Also, the right-hand sides of the equations (5.10)-(5.13) do not depend upon $\xi$ and they are bounded in $\tau$, with small bounds when $\eta, \rho$ and $\boldsymbol{w}^{0,0}$ are small.

In order to apply a center manifold reduction, we first have to modify the nonlinear terms. Let $\chi_{1}$ be an odd, smooth cut-off function defined on $[0, \infty)$ such that $\chi_{1}^{\prime} \geqslant 0, \chi_{1}(r)=r$ for $r \leqslant \delta$, and $\chi_{1}(r) \equiv 2 \delta$ for $r \geqslant 3 \delta$. We replace the right sides of the equations for $\xi_{x}$ and $\tau_{x}$ by $\chi_{1}(\cdot)$, with argument given by the original vector field. The resulting vector field coincides with the original vector field in a neighborhood of the modulated wave, is globally bounded with small bound, and has a small Lipschitz constant, for small $\delta, \eta, \rho$, and $\boldsymbol{w}$. In the equations for $\eta, \rho$, and $\boldsymbol{w}$, we multiply all nonlinear expressions in $\eta, \rho$, and $\boldsymbol{w}$ with a smooth cut-off function $\chi_{0}(|\eta|+|\rho|+\|\boldsymbol{w}\|)$, where $\chi_{0}(s)=1$ for $0 \leqslant s \leqslant \delta$ and $\chi_{0}(s)=0$ for $s \geqslant 2 \delta$ with bounds $0 \geqslant \chi_{0}^{\prime} \geqslant-2 / \delta$. Note that the cut-off in $\eta, \rho, \boldsymbol{w}$ preserves the action of the translations in space $\xi$ and time $\tau$

$$
\xi \mapsto \xi+\xi_{0}, \quad \tau \mapsto \tau+\tau_{0} .
$$

As a result, we find a system of equations of the form 


$$
\begin{aligned}
\xi_{x} & =g_{\xi}(\tau, \eta, \rho, \boldsymbol{w}), \\
\tau_{x} & =g_{\tau}(\tau, \eta, \rho, \boldsymbol{w}), \\
\eta_{x} & =g_{\eta}(\tau, \eta, \rho, \boldsymbol{w}), \\
\rho_{x} & =g_{\rho}(\tau, \eta, \rho, \boldsymbol{w}), \\
\boldsymbol{w}_{x} & =\mathcal{A}_{*}^{0, \tau} \boldsymbol{w}+g_{\boldsymbol{w}}(\tau, \eta, \rho, \boldsymbol{w}),
\end{aligned}
$$

where the nonlinearities $g_{j}, j=\xi, \tau, \eta, \rho, \boldsymbol{w}$ are small, bounded, with small Lipschitz constant. Since $\boldsymbol{w}(0)$ belongs to the smooth fiber bundle (id $\left.-P^{0, \tau}\right) Y$ and this space depends upon $\tau$, we cannot apply a center manifold reduction directly to the system (5.10)-(5.13). Therefore, we artificially augment the equation and allow for values $\boldsymbol{w}(0) \in Y$. Note that the linearized $\boldsymbol{w}$-equation possesses a four-dimensional center-eigenspace in the artificial directions (stemming from the generalized kernel of $\mathcal{A}_{*}$ ), such that we expect to find an eight-dimensional centermanifold.

The proof for the existence of an invariant manifold is now very similar to the construction of a slow manifold in singular perturbation problems and we omit the lengthy details; see [49] for a very detailed proof. Main ingredient is the existence of uniform exponential dichotomies for the linearized equation

$$
\boldsymbol{w}_{x}=\mathcal{A}_{*}^{0, \tau} \boldsymbol{w}
$$

for functions $\tau(x)$ with sup $\left|\tau_{x}\right|<\delta$, small; see [54, Theorem 7].

A fixed point argument provides us with an eight-dimensional center manifold for the artificially augmented system (5.16)-(5.20) with $w \in Y$. Since the cut-off preserved the symmetry action (5.15), the resulting centermanifold is invariant under this action. Since the action of the group on the tangent vectors is smooth, it is smooth on the smooth center-manifold. Note that the tangent space of the center-manifold contains at each individual point $\boldsymbol{q}_{*}^{\xi, \tau}$ the complementary artificial subspace of the $\boldsymbol{w}$-equation, given by the generalized kernel of $\mathcal{A}_{*}^{0, \tau}$. In particular, the smooth center-manifold and the smooth (invariant) fiber-bundle given by $P^{0, \tau} \boldsymbol{w}=0$ intersect transversely as smooth manifolds, such that their intersection is again a smooth manifold, invariant by the group action (5.15). We claim that the intersection is actually invariant under the flow on the reduced, "true" manifold. To see this, note that (5.10)-(5.13) defines a smooth vector field on the center-manifold which is, by definition of a center manifold tangent to the center eigenspace, and, by construction, tangent to the fiber bundle $P^{0, \tau} \boldsymbol{w}=0$. It is therefore tangent to the "true" center manifold and the flow on the artificially augmented center manifold must leave the true center manifold invariant. The "true" center manifold is tangent to the subspace parameterized by $\xi, \tau, \eta$, and $\rho$. The invariance under translations in space $\xi$ and time $\tau$ implies that the reduced vector field on the center-manifold is independent of $\xi$ and $\tau$. Moreover, the reversibility of the equation is preserved and acts via $\eta \mapsto-\eta$ and $\rho \mapsto-\rho$.

We compute the coefficients of the lowest order terms in this reduced system and find

$$
\begin{aligned}
\eta_{x}= & -\left\langle q_{* y}^{\mathrm{ad}}, \partial_{y y} q_{*}\right) \eta^{2}-2\left\langle q_{* y}^{\mathrm{ad}}, \partial_{y t} q_{*}\right\rangle \eta \rho-\left\langle q_{* y}^{\mathrm{ad}}, \partial_{t t} q_{*}\right\rangle \rho^{2} \\
& -\left\langle q_{* y}^{\mathrm{ad}}, D^{-1} \partial_{y} q_{*}\right\rangle\left(c-c_{*}\right)+\left\langle q_{* y}^{\mathrm{ad}}, D^{-1} \partial_{t} q_{*}\right\rangle\left(\omega-\omega_{*}\right), \\
\rho_{x}= & -\left\langle q_{* t}^{\mathrm{ad}}, \partial_{y y} q_{*}\right\rangle \eta^{2}-2\left\langle q_{* t}^{\mathrm{ad}}, \partial_{y t} q_{*}\right\rangle \eta \rho-\left\langle q_{* t}^{\mathrm{ad}}, \partial_{t t} q_{*}\right\rangle \rho^{2} \\
& -\left\langle q_{* t}^{\mathrm{ad}}, D^{-1} \partial_{y} q_{*}\right\rangle\left(c-c_{*}\right)+\left\langle q_{* t}^{\mathrm{ad}}, D^{-1} \partial_{t} q_{*}\right\rangle\left(\omega-\omega_{*}\right) .
\end{aligned}
$$

As in the case of travelling waves, Section 2, we have

$$
\left\langle q_{* i}^{\mathrm{ad}}, \partial_{y} q_{* j}\right\rangle=-\frac{c_{*}}{2}\left\langle q_{* i}^{\mathrm{ad}}, D^{-1} q_{* j}\right\rangle, \quad i, j \in\{y, t\},
$$

and the scalar products in right-hand side are calculated from the two-dimensional, linear dispersion relation involving $\Lambda_{\mathrm{d}}(k)$,

$$
\left(\begin{array}{ll}
d_{11} & d_{12} \\
d_{21} & d_{22}
\end{array}\right)=\left(\begin{array}{cc}
\left\langle q_{* y}^{\mathrm{ad}}, D^{-1} q_{* y}\right\rangle & \left\langle q_{* t}^{\mathrm{ad}}, D^{-1} q_{* y}\right\rangle \\
\left\langle q_{* y}^{\mathrm{ad}}, D^{-1} q_{* t}\right\rangle & \left\langle q_{* t}^{\mathrm{ad}}, D^{-1} q_{* t}\right\rangle
\end{array}\right)=-2\left(\Lambda_{\mathrm{d}}^{\prime \prime}(0)\right)^{-1} .
$$


We rewrite the system as

$$
\frac{\mathrm{d}}{\mathrm{d} x}\left(\begin{array}{c}
\eta \\
\rho
\end{array}\right)=\left(\begin{array}{ll}
d_{11} & d_{21} \\
d_{12} & d_{22}
\end{array}\right)\left(\begin{array}{c}
\frac{c_{*}}{2} \eta^{2}-c_{*} \alpha_{1} \rho^{2}-\left(c-c_{*}\right) \\
c_{*} \eta \rho-c_{*} \alpha_{2} \rho^{2}+\left(\omega-\omega_{*}\right)
\end{array}\right)
$$

in which

$$
\alpha_{1}=\frac{\left\langle q_{* y}^{\mathrm{ad}}, \partial_{t t} q_{*}\right\rangle d_{22}-\left\langle q_{* t}^{\mathrm{ad}}, \partial_{t t} q_{*}\right\rangle d_{21}}{c_{*}\left(d_{11} d_{22}-d_{12} d_{21}\right)}, \quad \alpha_{2}=\frac{\left\langle q_{* t}^{\mathrm{ad}}, \partial_{t t} q_{*}\right\rangle d_{11}-\left\langle q_{* y}^{\mathrm{ad}}, \partial_{t t} q_{*}\right\rangle d_{12}}{c_{*}\left(d_{11} d_{22}-d_{12} d_{21}\right)} .
$$

At $c=c_{*}$ and $\omega=\omega_{*}$ we find to leading order a quadratic system in which the origin is typically an isolated equilibrium. Quadratic systems in the plane have been classified by Markus [43]. For certain values of the coefficients such systems possess homoclinic solutions which decay algebraically as $|x| \rightarrow \infty$. These homoclinics correspond to holes in the reaction-diffusion system. In Section 5.2 we show that holes exist precisely when $\alpha_{2}^{2}<2 \alpha_{1}$. In particular, this will prove Theorem 5 .

At $\omega=\omega_{*}$ the reduced system possess two nontrivial equilibria for $c>c_{*}$, which correspond to rotations of the modulated front, as in the case discussed in Section 2,

$$
\eta_{ \pm}=\mp\left(\frac{c^{2}-c_{*}^{2}}{c^{2}}\right)^{1 / 2}, \quad \rho_{ \pm}=0 .
$$

In addition to this pair of equilibria we find another pair of equilibria $\left(\tilde{\eta}_{ \pm}, \tilde{\rho}_{ \pm}\right)$given at lowest order by

$$
\tilde{\eta}_{ \pm}=\alpha_{2} \tilde{\rho}_{ \pm}, \quad \tilde{\rho}_{ \pm}= \pm\left(\frac{2\left(c-c_{*}\right)}{c_{*}\left(\alpha_{2}^{2}-2 \alpha_{1}\right)}\right)^{1 / 2} .
$$

Therefore, if $\alpha_{2}^{2}<2 \alpha_{1}$ the reduced system has two equilibria for any $c \neq c_{*}$, and, if $\alpha_{2}^{2}>2 \alpha_{1}$ it has four equilibria for $c>c_{*}$ and no equilibria for $c<c_{*}$. Notice that at $c=c_{*}$ holes exist in the first case, and do not exist in the latter case. In both cases, the equilibrium $\left(\eta_{-}, 0\right)$ is a source and $\left(\eta_{+}, 0\right)$ a sink. Therefore, we find a one parameter family of heteroclinic orbits connecting $\left(\eta_{-}, 0\right)$ as $x \rightarrow-\infty$ with $\left(\eta_{+}, 0\right)$ as $x \rightarrow \infty$. These heteroclinics correspond to interior corners in the reaction-diffusion system, just as in the case of travelling fronts in Section 2.

The additional equilibria $\left(\tilde{\eta}_{ \pm}, \tilde{\rho}_{ \pm}\right)$correspond to rotated modulated fronts with a periodic modulation in $x$ direction. In fact, for any "average angle" $\eta$ and any transverse modulational wavenumber $\rho$, we can find a speed $c$ and a frequency $\omega$ such that there exists a modulated front with the prescribed values of $\eta$ and $\rho$. Fixing $\eta=0$ factors the rotational invariance due to isotropy of diffusion, which gives a family of rotated waves together with a given modulated wave. As to leading order, the nonlinear dispersion relation of these waves is given by $(c, \omega)(\rho)=$ $\left(c_{*}, \omega_{*}\right)+c_{*}\left(-\alpha_{1}, \alpha_{2}\right) \rho^{2}$. We expect these waves to be linearly unstable in case $\alpha_{2}^{2}<2 \alpha_{1}$, where the characteristics of an associated transport equation are complex; see Appendix B. For our problem, these two additional equilibria allow for constructing further corner defects. However, we do not investigate this possibility in the present paper.

\subsection{Existence of holes}

We show that holes exist under certain algebraic conditions on the coefficients of the reduced system (5.21), (5.22).

We set $c=c_{*}, \omega=\omega_{*}$, and the reduced system becomes

$$
\frac{\mathrm{d}}{\mathrm{d} x}\left(\begin{array}{c}
\eta \\
\rho
\end{array}\right)=c_{*}\left(\begin{array}{ll}
d_{11} & d_{21} \\
d_{12} & d_{22}
\end{array}\right)\left(\begin{array}{c}
\frac{1}{2} \eta^{2}-\alpha_{1} \rho^{2} \\
\eta \rho-\alpha_{2} \rho^{2}
\end{array}\right)+\mathrm{O}\left((|\eta|+|\rho|)^{3}\right) .
$$

The stability Hypothesis 5.3 implies that the matrix $\left(d_{i j}\right)$ is positive definite in the sense that the spectrum consists of eigenvalues with strictly positive real part. For $\alpha_{2}^{2}=2 \alpha_{1}$ the leading-order, quadratic part possesses a line of equilibria and no other bounded solutions. In order to detect small bounded solutions, cubic terms have to be taken into account. We therefore assume from now on that $\alpha_{2}^{2} \neq 2 \alpha_{1}$. 
We are interested in homoclinic orbits to the origin. At lowest order, we have a homogeneous, quadratic differential equation in the plane. Markus [43] classified all planar quadratic differential equations. He associated a commutative, two-dimensional, real linear algebra with the vector field such that vector fields are affine equivalent if, and only if, the associated algebras are isomorphic. Since we have to take higher-order terms into account, as well, we pursue a slightly different strategy. We introduce polar coordinates which automatically factor the leadingorder scaling symmetry. As a result, we recover Markus' phase portraits for the quadratic system and show at the same time that the homoclinic orbits persist in the full reduced system (5.23).

Proposition 5.4. Assume that the matrix $\left(d_{i j}\right)$ is positive definite and that the coefficients $\alpha_{1}$ and $\alpha_{2}$ of the reduced system (5.23) satisfy $\alpha_{2}^{2}<2 \alpha_{1}$. There is $\varepsilon_{0}>0$ such that (5.23) possess two families of homoclinic orbits,

$$
\left(\eta_{\varepsilon}^{ \pm}(x), \rho_{\varepsilon}^{ \pm}(x)\right)=\left( \pm \varepsilon \eta_{o}( \pm \varepsilon x), \pm \varepsilon \rho_{o}( \pm \varepsilon x)\right)+\mathrm{O}\left(\varepsilon^{2}\right)
$$

for $\varepsilon \in\left(0, \varepsilon_{0}\right)$, in which $\left(\eta_{o}, \rho_{o}\right)$ decay like $\mathrm{O}(1 /|x|)$ as $|x| \rightarrow \infty$. For $\alpha_{2}^{2}>2 \alpha_{1}$ there are no nontrivial, small, bounded solutions to (5.23).

The homoclinic orbits found in this proposition correspond to holes in the reaction-diffusion system (2.1). The algebraic decay of $\eta$ and $\rho$ generates a logarithmic divergence of the position $\xi$ and the temporal phase $\tau$ of the front. Both speed and frequency are given by the primary planar modulated front.

We will show in Appendix B that the dynamics of modulated fronts with temporal phase and $y$-position slowly varying in the transverse direction $x$ and time $t$, can be formally described by a system of viscous conservation laws for $\eta$ and $\rho$. The dynamical behavior of such a system on large spatial scales depends crucially on the eigenvalues of the flux function: real, distinct eigenvalues correspond to the strictly hyperbolic case, where global existence in time can be expected. Complex eigenvalues yield an ill-posed Cauchy-problem in the zero-viscosity limit, with an immediate Hadamard instability causing blow-up. Interestingly, the algebraic condition on the coefficients of the reduced system in Proposition 5.4 which ensures the existence of homoclinic orbits turns out to be the condition for complex characteristics in the zero-viscosity regime. More precisely, we show in the appendix that the remaining system of conservation laws has real characteristics if $\alpha_{2}^{2}>2 \alpha_{1}$ and complex characteristics for the opposite inequality. A similar phenomenon has been observed in [8], where wave propagation in the focusing NLS-equation is studied. In the semiclassical limit, phase and amplitude evolution is governed by transport equations with complex characteristic.

Proof of Proposition 5.4. Without loss of generality we set $c_{*}=1$ and assume that $d_{12} \geqslant 0$ (otherwise, we can rescale $x$ to have $c_{*}=1$, and change $\rho \rightarrow-\rho$ to find the same system with coefficients $-d_{12},-d_{21},-\alpha_{2}$ ).

Recall that $\alpha_{2}^{2} \neq 2 \alpha_{1}$, so that the origin is an isolated fixed point. Then the quadratic system possess at least one ray solution,

$$
\eta(x)=-\frac{\eta_{*}}{x}, \quad \rho(x)=-\frac{\rho_{*}}{x},
$$

with constants $\eta_{*}$ and $\rho_{*}$ satisfying

$$
\left(\begin{array}{c}
\eta_{*} \\
\rho_{*}
\end{array}\right)=\left(\begin{array}{ll}
d_{11} & d_{21} \\
d_{12} & d_{22}
\end{array}\right)\left(\begin{array}{c}
\frac{1}{2} \eta_{*}^{2}-\alpha_{1} \rho_{*}^{2} \\
\eta_{*} \rho_{*}-\alpha_{2} \rho_{*}^{2}
\end{array}\right) .
$$

Homoclinic orbits of the quadratic system approach the origin along ray solutions; see Fig. 7. In particular, they decay algebraically, $(\eta, \rho)(x)=\mathrm{O}(1 /|x|)$ as $|x| \rightarrow \infty$.

First notice that a necessary condition for the existence of homoclinic orbits is that both quadratic functions in the right-hand side of (5.23),

$$
d_{11}\left(\frac{1}{2} \eta^{2}-\alpha_{1} \rho^{2}\right)+d_{21}\left(\eta \rho-\alpha_{2} \rho^{2}\right)
$$



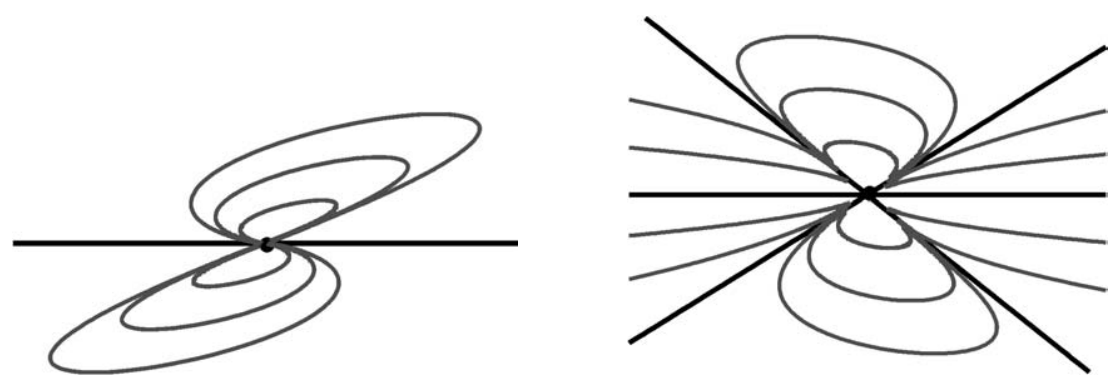

Fig. 7. Typical homoclinic orbits of the quadratic system in the case of one ray solution (left) and three ray solutions (right).

and

$$
d_{12}\left(\frac{1}{2} \eta^{2}-\alpha_{1} \rho^{2}\right)+d_{22}\left(\eta \rho-\alpha_{2} \rho^{2}\right),
$$

change sign. Indeed, if, for example, the first function has constant sign, then the derivative of $\eta$ has constant sign such that the $\eta$-component of solutions is monotone excluding homoclinic orbits in this case. Similarly, the sign of the second function encodes monotonicity of the $\rho$-component. A straightforward calculation shows that the necessary and sufficient conditions for these two functions to change sign are

$$
\left(d_{2 j}+\alpha_{2} d_{1 j}\right)^{2}+d_{1 j}^{2}\left(2 \alpha_{1}-\alpha_{2}^{2}\right)>0, \quad j=1,2 .
$$

Notice that both inequalities hold if $\alpha_{2}^{2}<2 \alpha_{1}$.

We therefore assume from now on that the coefficients of the reduced system satisfy (5.25). Moreover, we assume that $d_{12}>0$, the case $d_{12}=0$ can be treated in a similar way. We introduce blow-up coordinates and replace $(\eta, \rho) \in \mathbb{R}^{2}$ by polar coordinates on $\mathbb{R}_{+} \times S^{1}$, thus blowing up the origin to a circle $\{0\} \times S^{1}$. We parameterize the circle by two directional blow-up charts $\left(\eta_{j}, \rho_{j}\right), j=1,2$, which we refer to as the 1-chart and the 2-chart. They correspond to stereographic projections of the angular coordinate, and are explicitly given by scaling invariants and equivariants

$$
\left(\eta_{1}, \rho_{1}\right)=\left(\frac{\eta}{\rho}, \rho\right), \quad\left(\eta_{2}, \rho_{2}\right)=\left(\eta, \frac{\rho}{\eta}\right) ;
$$

see Fig. 8, for an illustration of the various charts in an example. It is a simple exercise to see that these charts smoothly parameterize $\mathbb{R}_{+} \times S^{1}$.

For $\eta_{1} \neq 0$ and $\rho_{2} \neq 0$, the coordinate change $\eta_{1}=1 / \rho_{2}, \rho_{1}=\eta_{2} \rho_{2}$, defines a diffeomorphic change of coordinates between the two charts. Note that strictly speaking, the above set of coordinates defines four charts in $\mathbb{R}_{+} \times S^{1}$, depending on whether $\rho_{1} \geqslant 0$ or $\rho_{1} \leqslant 0$ in the 1 -chart and whether $\eta_{2} \geqslant 0$ or $\eta_{2} \leqslant 0$ in the 2 -chart. Since the two variants of the 1- and 2-charts give algebraically equivalent vector fields, we do not formally distinguish between them. In these new blow-up coordinates the system becomes

$$
\begin{aligned}
& \eta_{1}^{\prime}=\rho_{1}\left(\left(d_{11}-d_{12} \eta_{1}\right)\left(\frac{1}{2} \eta_{1}^{2}-\alpha_{1}\right)+\left(d_{21}-d_{22} \eta_{1}\right)\left(\eta_{1}-\alpha_{2}\right)\right)+\mathrm{O}\left(\left|\rho_{1}\right|^{2}\right), \\
& \rho_{1}^{\prime}=\rho_{1}^{2}\left(d_{12}\left(\frac{1}{2} \eta_{1}^{2}-\alpha_{1}\right)+d_{22}\left(\eta_{1}-\alpha_{2}\right)\right)+\mathrm{O}\left(\left|\rho_{1}\right|^{3}\right),
\end{aligned}
$$

and

$$
\begin{aligned}
& \eta_{2}^{\prime}=\eta_{2}^{2}\left(d_{11}\left(\frac{1}{2}-\alpha_{1} \rho_{2}^{2}\right)+d_{21}\left(1-\alpha_{2} \rho_{2}\right) \rho_{2}\right)+\mathrm{O}\left(\left|\eta_{2}\right|^{3}\right) \\
& \rho_{2}^{\prime}=\eta_{2}\left(\left(d_{12}-d_{11} \rho_{2}\right)\left(\frac{1}{2}-\alpha_{1} \rho_{2}^{2}\right)+\left(d_{22}-d_{21} \rho_{2}\right)\left(1-\alpha_{2} \rho_{2}\right) \rho_{2}\right)+\mathrm{O}\left(\left|\eta_{2}\right|^{2}\right)
\end{aligned}
$$




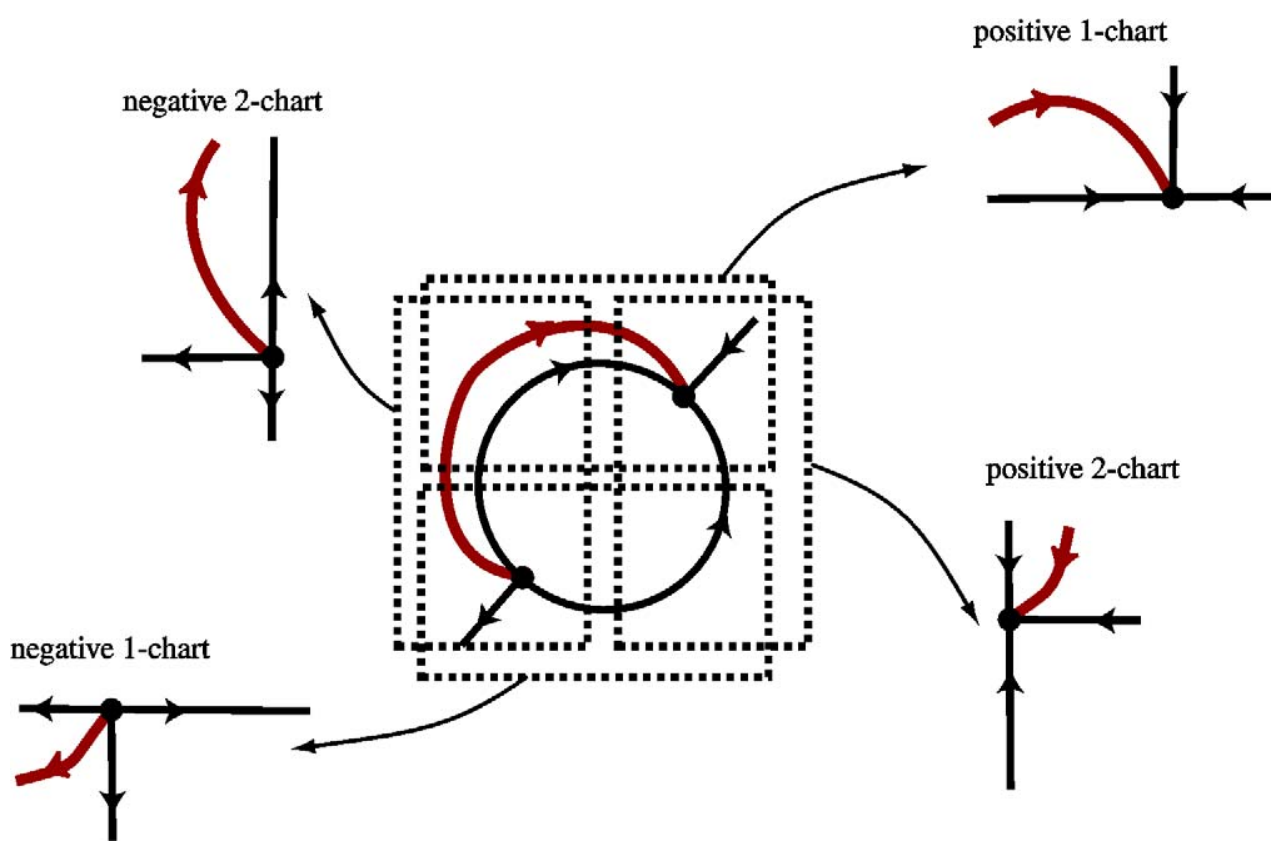

Fig. 8. Picture of the blow-up phase portrait with the positive and negative 1- and 2-charts. The coordinate axes are $\eta, \eta_{j}$ horizontal and $\rho, \rho_{j}$ vertical. The inner circle represents the equilibrium $\{0\} \times S^{1}$. The specific dynamics exemplify the existence of a hole solution in the case where $f_{1}$, defined in (5.26) possesses only one real root.

respectively, in which ${ }^{\prime}=\mathrm{d} / \mathrm{d} x$. Both systems have an Euler multiplier given by $\rho_{1}$ and $\eta_{2}$, respectively. We therefore reparameterize spatial time $x$ by introducing the new independent variables $z_{j}$ implicitly through $\mathrm{d} z_{1}=$ $\rho_{1} \mathrm{~d} x$ in the first chart, and by $\mathrm{d} z_{2}=\eta_{2} \mathrm{~d} x$ in the second chart. At lowest order we obtain the systems

$$
\dot{\eta}_{1}=f_{1}\left(\eta_{1}\right), \quad \dot{\rho}_{1}=\rho_{1} g_{1}\left(\eta_{1}\right),
$$

and

$$
\dot{\eta}_{2}=\eta_{2} g_{2}\left(\rho_{2}\right), \quad \dot{\rho}_{2}=f_{2}\left(\rho_{2}\right),
$$

where

$$
\begin{aligned}
& f_{1}\left(\eta_{1}\right)=\left(d_{11}-d_{12} \eta_{1}\right)\left(\frac{1}{2} \eta_{1}^{2}-\alpha_{1}\right)+\left(d_{21}-d_{22} \eta_{1}\right)\left(\eta_{1}-\alpha_{2}\right), \\
& g_{1}\left(\eta_{1}\right)=d_{12}\left(\frac{1}{2} \eta_{1}^{2}-\alpha_{1}\right)+d_{22}\left(\eta_{1}-\alpha_{2}\right),
\end{aligned}
$$

and

$$
\begin{aligned}
& f_{2}\left(\rho_{2}\right)=\left(d_{12}-d_{11} \rho_{2}\right)\left(\frac{1}{2}-\alpha_{1} \rho_{2}^{2}\right)+\left(d_{22}-d_{21} \rho_{2}\right)\left(1-\alpha_{2} \rho_{2}\right) \rho_{2}, \\
& g_{2}\left(\rho_{2}\right)=d_{11}\left(\frac{1}{2}-\alpha_{1} \rho_{2}^{2}\right)+d_{21}\left(1-\alpha_{2} \rho_{2}\right) \rho_{2} .
\end{aligned}
$$

Here, the dots stand for $\mathrm{d} / \mathrm{d} z_{1}$ in the first system and for $\mathrm{d} / \mathrm{d} z_{2}$ in the second system. Note that when time $x$ increases the scaled time $z_{1}$ (resp. $z_{2}$ ) increases if $\rho_{1}>0$ (resp. $\left.\eta_{2}>0\right)$ and decreases if $\rho_{1}<0$ (resp. $\eta_{2}<0$ ). The lines $\rho_{1}=0$ and $\eta_{2}=0$, respectively, correspond to the singular circle $\{0\} \times S^{1}$ and are therefore invariant under 
the flows of (5.26) and (5.27), respectively. If $\left(\eta_{* 1}, 0\right)$ is a fixed point of (5.26), then the line $\eta_{1}=\eta_{* 1}$ is invariant for the flow to this leading-order part of the reduced equation. A similar statement holds for the 2-chart (5.27).

In our directional blow-up coordinates, the ray solutions $\left(-\eta_{*} / x,-\rho_{*} / x\right)$ of the quadratic system (5.23) are perpendicular to the $\eta_{1}$ - and the $\rho_{2}$-axis, respectively. Ray solutions therefore correspond to invariant lines $\eta_{1}=$ $\eta_{*} / \rho_{*}$ or $\rho_{2}=\rho_{*} / \eta_{*}$, or, equivalently, the equilibria $\left(\eta_{*} / \rho_{*}, 0\right)$ in the 1 -chart and the equilibria $\left(0, \rho_{*} / \eta_{*}\right)$ in the 2-chart. Homoclinic orbits of (5.23) are in one-to-one correspondence with heteroclinic connections between the singular equilibria of (5.26) and (5.27) on the singular circle $\{0\} \times S^{1}$. The number of equilibria of (5.26) and (5.27) is given by the number of zeros of $f_{1}$ and $f_{2}$, and they are in one-to-one correspondence with the ray solutions of the quadratic system.

The dynamics of the systems (5.26) and (5.27) are qualitatively determined by the equilibria on the circle, that is, by the roots of $f_{1}$ and $f_{2}$, and the signs of $g_{1}$ and $g_{2}$ at these roots, which in turn can be inferred from the position of the roots of $g_{j}$ relative to the roots of $f_{j}$. Since we assumed $d_{12}>0$ and concluded (5.25), $g_{1}$ possesses two real roots $\eta_{01}<\eta_{02}$, where

$$
f_{1}\left(\eta_{0 j}\right)=\frac{d_{11} d_{22}-d_{12} d_{21}}{d_{12}}\left(\alpha_{2}-\eta_{0 j}\right) .
$$

Since $g_{1}\left(\alpha_{2}\right)=d_{12}\left(\alpha_{2}^{2}-2 \alpha_{1}\right) / 2$, we have $\eta_{01}<\alpha_{2}<\eta_{02}$ if $\alpha_{2}^{2}<2 \alpha_{1}$, and $\alpha_{2}<\eta_{01}<\eta_{02}$ or $\eta_{01}<\eta_{02}<\alpha_{2}$ if $\alpha_{2}^{2}>2 \alpha_{1}$. We conclude that $f_{1}\left(\eta_{01}\right)>0$ and $f_{1}\left(\eta_{02}\right)<0$ if $\alpha_{2}^{2}<2 \alpha_{1}$, and that $f_{1}\left(\eta_{01}\right)$ and $f_{1}\left(\eta_{02}\right)$ have the same sign if $\alpha_{2}^{2}>2 \alpha_{1}$.

We now distinguish several cases depending upon the number of real roots of $f_{1}$, that is, the number of equilibria of (5.26). Note that, since $d_{12}>0$, the number of equilibria on the singular circle is precisely twice the number of roots of $f_{1}$ (counting each root for the negative and positive 1-chart). The shape of the polynomial $f_{1}$ and the relative position of its roots and of $\eta_{01}$ and $\eta_{02}$ are plotted in the case of three real roots in Fig. 9 , if $\alpha_{2}^{2}<2 \alpha_{1}$, and Fig. 11, if $\alpha_{2}^{2}>2 \alpha_{1}$, and in the case of one real root in Fig. 13. The corresponding phase portraits of (5.26) are shown in Figs. 10, 12 and 14, respectively. These phase portraits indicate that heteroclinic connections exist only in the case $\alpha_{2}^{2}<2 \alpha_{1}$.

In order to conclude the robust existence or nonexistence of hole solutions, first note that all solutions asymptotic to the singular circle in forward or backward spatial time converge to precisely one equilibrium on the circle. Next note that the singular equilibria are connected by singular heteroclinic orbits in the singular circle $\{0\} \times S^{1}$. If
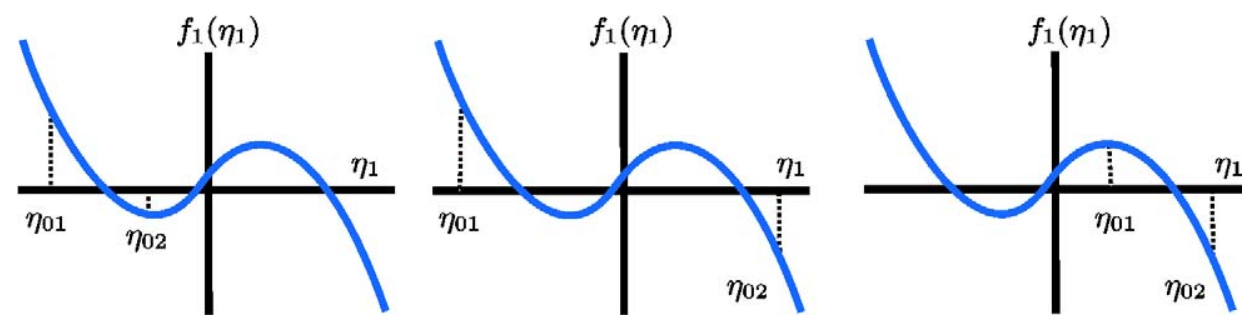

Fig. 9. The nullclines of $f_{1}$ with the zeroes $\eta_{01}$ and $\eta_{02}$ of $g_{1}$ in the case of three real roots of $f_{1}$ and $\alpha_{2}^{2}<2 \alpha_{1}$.
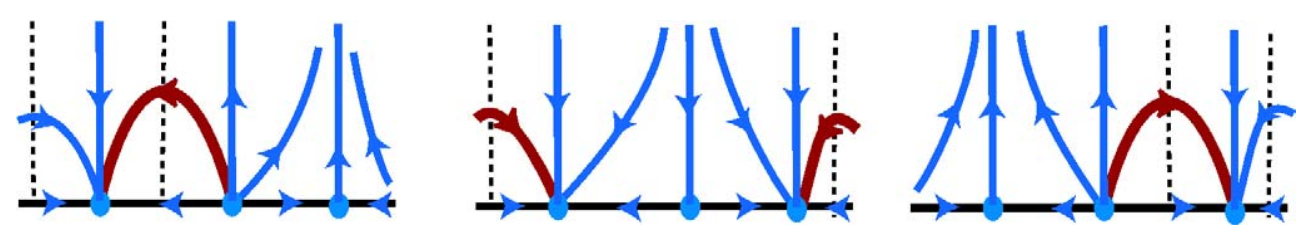

Fig. 10. Phase portraits in the $\left(\eta_{1}, \rho_{1}\right)$-plane in case $\alpha_{2}^{2}<2 \alpha_{1}$ and three real roots of $f_{1}$. The dashed lines indicate the zeroes $\eta_{01}$ and $\eta_{02}$ of $g_{1}$. The bold orbits are heteroclinic orbits in the blow-up coordinates and therefore yield homoclinic, hole solutions. 

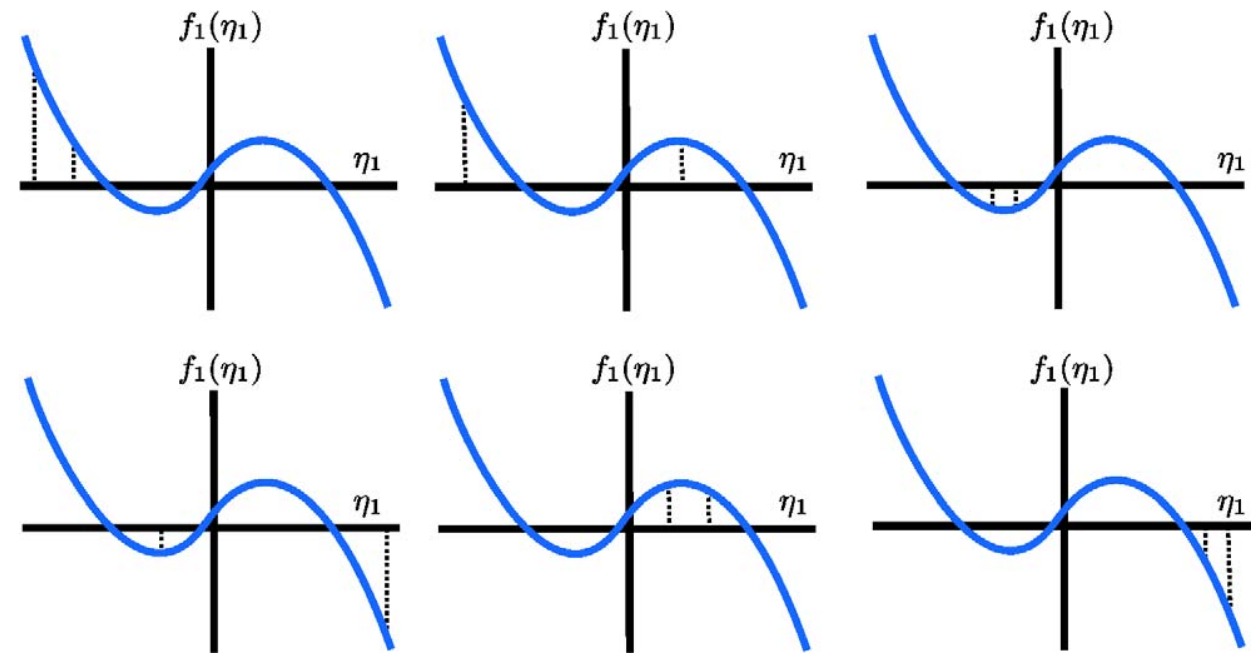

Fig. 11. The nullclines of $f_{1}$ with the zeroes $\eta_{01}$ and $\eta_{02}$ of $g_{1}$ in the case of three real roots of $f_{1}$ and $\alpha_{2}^{2}>2 \alpha_{1}$. Like in Fig. 9, the dashed lines indicate the location of the zeroes of $g_{1}$.
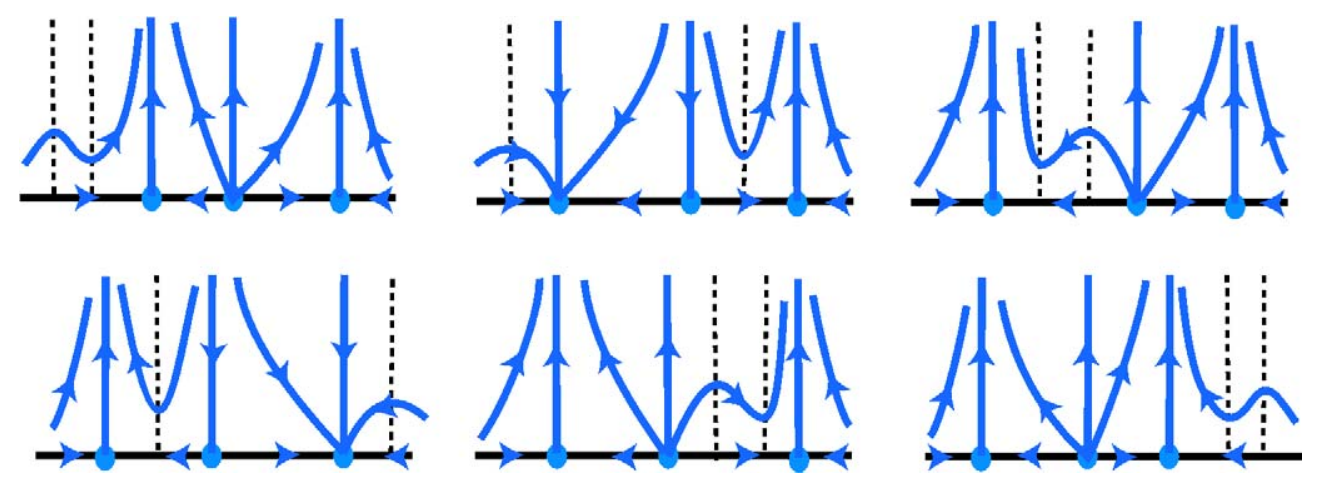

Fig. 12. Phase portraits in the $\left(\eta_{1}, \rho_{1}\right)$-plane in case $\alpha_{2}^{2}>2 \alpha_{1}$ and three real roots of $f_{1}$. The dashed lines indicate the zeroes $\eta_{01}$ and $\eta_{02}$ of $g_{1}$. There do not exist nontrivial bounded solutions.
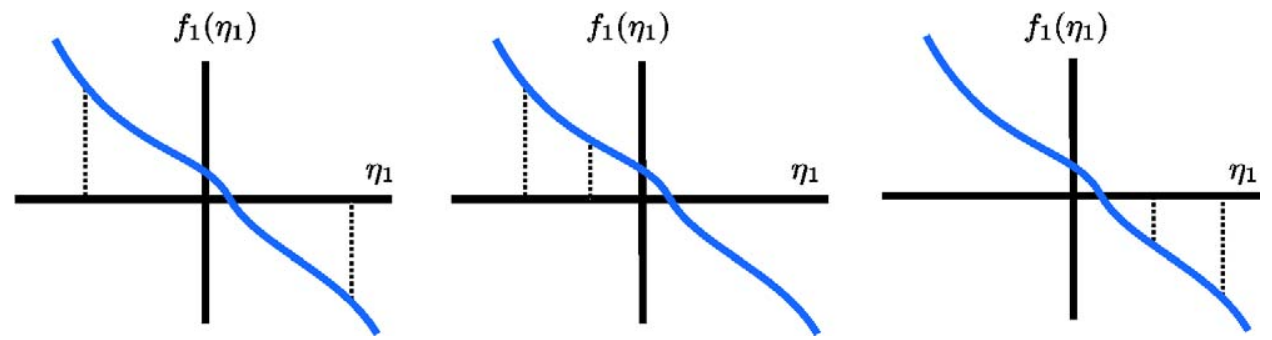

Fig. 13. The nullclines of $f_{1}$ with the zeroes $\eta_{01}$ and $\eta_{02}$ of $g_{1}$ in the case of one real root of $f_{1}$. In the left picture, we have $\alpha_{2}^{2}<2 \alpha_{1}$, in the middle and in the right picture, we have $\alpha_{2}^{2}>2 \alpha_{1}$. Like in Fig. 9, the dashed lines indicate the location of the zeroes of $g_{1}$. 

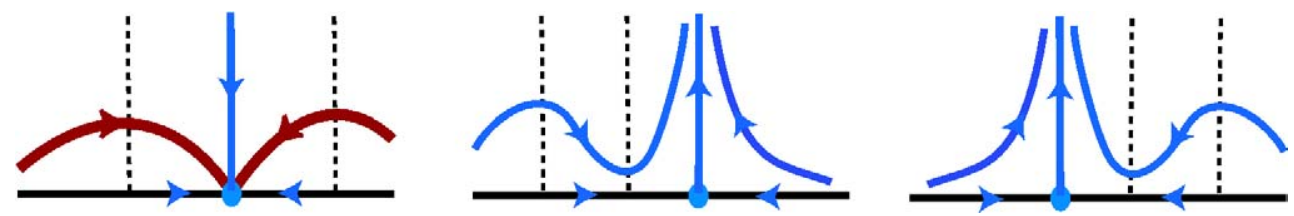

Fig. 14. Phase portraits in the $\left(\eta_{1}, \rho_{1}\right)$-plane in case of one real root of $f_{1}$. Again, $\alpha_{2}^{2}<2 \alpha_{1}$ in the left picture and $\alpha_{2}^{2}>2 \alpha_{1}$ in the middle and right picture. The dashed lines indicate the zeroes $\eta_{01}$ and $\eta_{02}$ of $g_{1}$. The bold orbits in the left picture are heteroclinic orbits in the blow-up coordinates and therefore yield homoclinic, hole solutions. There are no nontrivial bounded solutions in the middle and in the right picture.

one of the equilibria is of saddle-type, the singular heteroclinic orbit is the locally unique trajectory approaching this equilibrium, and there are no solutions outside the singular circle approaching this given equilibrium nearby. In particular, small bounded solutions can only exist as heteroclinic orbits between singular equilibria which are sources or sinks, respectively. Since the set of heteroclinic orbits between a source and a sink in the plane forms an open subset of the plane, we can infer the existence of a family of small heteroclinic orbits close to the singular heteroclinic, which shows existence of holes precisely in the case when two neighboring equilibria on the circle of source or sink type, respectively. Note, however, that we have to be careful when passing from the positive 1-chart to the negative 1-chart since time is reversed by the negative Euler multiplier $\rho_{1}$ in the negative 1-chart, and similarly for the 2-chart.

As an example, consider the case of one equilibrium $\left(\eta_{* 1}, 0\right)$ in the positive one-chart, illustrated in Fig. 8. In the positive 1-chart, the equilibrium is a source precisely when $\alpha_{2}^{2}<2 \alpha_{1}$, and a saddle, otherwise. The second equilibrium on the singular circle is located on the opposite side, with reversed stability properties due to the negative Euler multiplier. The singular heteroclinic, given by the arc of the singular circle joining the two equilibria, is accompanied by a family of heteroclinics which form the (necessarily transverse) intersection of stable and unstable manifolds of the two singular equilibria.

To conclude the proof of the proposition, we remark that the representation of holes (5.24) is due to the invariance of the quadratic part of (5.23) under the scaling

$$
x=\frac{\tilde{x}}{a}, \quad \eta=a \tilde{\eta}, \quad \rho=a \tilde{\rho},
$$

for $a \in \mathbb{R}$, and due to reversibility acting through

$$
x \mapsto-x, \quad \eta \mapsto-\eta, \quad \rho \mapsto-\rho .
$$

Remark 5.5. We emphasize that hole solutions are not symmetric in $x$ (that is, invariant under the reversibility operation $(\eta, \rho) \mapsto-(\eta, \rho))$. In particular, there typically exists a robust family of hole solutions, parameterized by their amplitude, which are not symmetric in $x$, but their velocity is still perpendicular to the $x$-axis. Note that the direction of propagation is chosen in a natural way to be the $y$-axis which is normal to the asymptotic tangent space of the interface. Indeed, since $\eta \rightarrow 0$ for $|x| \rightarrow \infty$, the tangent space to the interface at $\pm \infty$ is the $x$-axis. In particular, the 1-parameter family of holes that we found cannot be viewed as a 1-parameter family of rotated interfaces.

Remark 5.6 (Nonexistence of holes for weakly pulsating fronts). If we assume that the modulated front $q_{*}(y, t)$ is a small perturbation of a planar travelling front (weakly pulsating front), holes generally do not exist. More precisely, assume that the reaction-diffusion system (2.1) possesses a planar travelling front $q_{* 0}(y)$, as in Section 2. Suppose that close to this travelling front there is a family of modulated fronts $q_{*}\left(y, \omega_{*}(\mu) t ; \mu\right)$ with speeds $c_{*}(\mu)=c_{*}+\mathrm{O}(\mu)$ and frequencies $\omega_{*}(\mu)=\omega_{*}+\mathrm{O}(\mu)$ in $t$, such that

$$
q_{*}\left(y, \omega_{*}(\mu) t ; \mu\right)=q_{* 0}(y)+\mu q_{1}\left(y, \omega_{*}(\mu) t ; \mu\right),
$$


for small $\mu$. Weakly pulsating fronts of this type are found, for example, near a planar travelling front $q_{* 0}$ which undergoes a Hopf bifurcation; see [44] for an example. For such a modulated front, the coefficients of the reduced system satisfy

$$
\left\langle q_{* y}^{\mathrm{ad}}, \partial_{y y} q_{*}\right\rangle=\mathrm{O}(1), \quad\left\langle q_{* y}^{\mathrm{ad}}, \partial_{y t} q_{*}\right\rangle=\mathrm{O}(\mu), \quad\left\langle q_{* y}^{\mathrm{ad}}, \partial_{t t} q_{*}\right\rangle=\mathrm{O}(\mu),
$$

and

$$
\left\langle q_{* t}^{\mathrm{ad}}, \partial_{y y} q_{*}\right\rangle=\mathrm{O}(1 / \mu), \quad\left\langle q_{* t}^{\mathrm{ad}}, \partial_{y t} q_{*}\right\rangle=\mathrm{O}(1), \quad\left\langle q_{* t}^{\mathrm{ad}}, \partial_{t t} q_{*}\right\rangle=\mathrm{O}(1)
$$

Therefore, the inequality $\alpha_{2}^{2}<2 \alpha_{1}$ is not verified for small $\mu$, and we can conclude that holes do not exist for weakly pulsating fronts.

\section{Discussion}

We have presented a framework for the study of weak localized corners in almost planar interface propagation. The results are formulated for general reaction-diffusion systems. Assumptions are only on existence of primary planar fronts, and on spectral stability or instability properties of these fronts. In the most simple case of the rigid propagation of a stable planar interface, we have found asymptotically stable interior corners, where two planar fronts collide at an angle $\varphi<\pi$; Sections 2 and 3. Long wavelength instabilities generate more complicated structures such as exterior corners and steps; Section 4. In oscillatory wave propagation, we showed that weakly localized holes can be embedded in a planar interface, such that the angles of the hole interface relative to the planar interface tend to zero on both sides of the hole; Section 5. Most of our results can be formally obtained by first deriving a transport equation and then looking for travelling-wave solutions of this transport equation. Our approach avoids the subtle questions involved with the validity of the approximation. On the other hand, the existence of certain corner defects, such as holes, seems to be intrinsically related to well-posedness properties of transport equations in the inviscid limit; Section 5.2. We conclude this paper mentioning a number of straightforward extensions to our approach, and pointing out some open problems.

We first comment on planar interfaces between not necessarily spatially homogeneous patterns. In [52], modulated fronts are constructed that invade a spectrally stable spatially periodic pattern, leaving a stable homogeneous state behind. More precisely, there exists a spatio-temporally periodic solution $q_{+}\left(\omega_{+} t-k_{+} y\right)$, with $q_{+}(\phi)=q_{+}(\phi+2 \pi)$, a spatially homogeneous state $q_{-}$, and a modulated front $q_{*}\left(y, \omega_{+} t\right)=q_{*}\left(y, \omega_{+} t+2 \pi\right)$ connecting these two states, that is,

$$
q_{*}(y, \tau) \rightarrow q_{+}\left(k_{+} y-\omega_{+} \tau\right) \quad \text { for } y \rightarrow+\infty, \quad q_{*}(y, \tau) \rightarrow q_{-} \quad \text { for } y \rightarrow-\infty,
$$

uniformly in $\tau$. If the group velocity $c_{\mathrm{g}}$ of the periodic pattern is negative, directed towards the interface, then the linearized operator $\mathcal{M}_{0}$ appearing in (5.5), typically possesses a one-dimensional kernel, only, in spaces with exponential weights with rates $\eta>0$, small, as defined in (2.32); see [54] for the notion of group velocities and spectra of interfaces between spatially periodic patterns. Going through the reduction steps, we therefore expect the existence of a two-dimensional center-manifold containing stable interior corners.

A similar situation arises for fronts invading symmetric, $x$-periodic patterns $q_{+}\left(k_{+} x\right)=q_{+}\left(k_{+} x+2 \pi\right)=$ $q_{+}\left(-k_{+} x\right)$,

$$
q_{*}(y, x) \rightarrow q_{+}\left(k_{+} x\right) \quad \text { for } y \rightarrow+\infty, \quad q_{*}(y, x) \rightarrow q_{-} \text {for } y \rightarrow-\infty,
$$

where $q_{*}(y, x)=q_{*}\left(y, x+2 \pi / k_{+}\right)$. Again, the spectrum of the linearization contains only a simple eigenvalue $\lambda=0$, when considered in spaces of exponentially localized functions as described above. We can therefore reduce to a two-dimensional center-manifold. Since the patterns depend explicitly on the spatial time-variable $x$ in a periodic fashion, the resulting flow on the center-manifold will be periodically forced. Still, the unfolding of the 
saddle-node bifurcation for the period map should yield the very same interior corners that we have found in the case of rigid front propagation.

For intermediate situations, where the propagation of the front takes an angle $\vartheta \notin\{0, \pi / 2\}$ relative to the orientation of the stripe pattern ahead of the front, the reduced equations would not be reversible anymore. In particular, the periodic patterns typically possess a nonzero group velocity in the direction tangential to the interface such that the generalized kernel in our spatial dynamics formulation would only be one-dimensional, with the planar front as the unique bounded solution in the one-dimensional center-manifold. Suppose for example that the angle between the contour lines of a roll pattern and the interface is $\vartheta$, such that the roll pattern is given by $q_{+}((\cos \vartheta) x-(\sin \vartheta) y)$, $q_{+}(\phi)=q_{+}(-\phi)$. The shear transformation $\xi=(\cos \vartheta) x-(\sin \vartheta) y$ allows us to look for rigidly propagating front solutions $q_{*}(y, \xi)=q_{*}\left(y, \xi+2 \pi / k_{+}\right)$invading the $y$-independent roll solution $q_{+}(\xi)$. However, although the roll pattern $q_{+}$is symmetric, the travelling front will typically not be symmetric since the defining equation for the interface

$$
D\left[\cos ^{2} \vartheta \partial_{\xi \xi}+\left(\partial_{y}-\sin \vartheta \partial_{\xi}\right)^{2}\right] u+c_{*}\left(\partial_{y}-\sin \vartheta \partial_{\xi}\right) u+f(u)=0,
$$

posed on functions with period $2 \pi / k_{+}$in $\xi$, is not symmetric in $\xi$. We can actually compute a transverse dispersion relation $\lambda(\nu)$, substituting the Ansatz $\mathrm{e}^{\nu \xi} v(y, \xi), v(y, \xi)=v\left(y, \xi+2 \pi / k_{+}\right)$, into the linearization about the front

$$
D\left[\cos ^{2} \vartheta\left(\partial_{\xi}+v\right)^{2}+\left(\partial_{y}-\sin \vartheta\left(\partial_{\xi}+v\right)\right)^{2}\right] v+c_{*}\left(\partial_{y}-\sin \vartheta\left(\partial_{\xi}+v\right)\right) v+f^{\prime}\left(q_{*}\right) v=\lambda v .
$$

Near $\lambda=v=0$, we typically find a tangential group velocity along the interface $c_{\mathrm{g}}^{\|}=\frac{\mathrm{d} \lambda}{\mathrm{d} v} \neq 0$ in $v=0$. If we denote by $\partial_{y} q_{*}$ and $q_{*}^{\text {ad }}$ the normalized eigenvectors in the kernel of the linearization and its adjoint, as constructed in Section 2, we find

$$
\frac{\mathrm{d} \lambda}{\mathrm{d} \nu}(0)=\left\langle\left(2 D\left(\partial_{\xi}-\beta \partial_{y}\right)-c_{*} \beta\right) \partial_{y} q_{*}, q_{*}^{\mathrm{ad}}\right\rangle,
$$

where we set $\beta=\sin \vartheta$ and scalar products are in $L^{2}(\mathbb{R} \times[0,2 \pi])$. The second term gives a contribution $c_{*} \sin \vartheta$ which is the purely geometrically transport induced from the normal speed of propagation under the shear transformation. The first term reflects the tangential dispersion relation; see also (2.25).

In order to find weak interior corners, we can now change to a $\xi$-comoving frame, introducing $\zeta=\xi-c_{\mathrm{g}}^{\|} t$ as a new variable. In the new variables, the front is a time-periodic, modulated wave, but, as a straightforward but tedious calculation shows, the dispersion relation in the tangential direction vanishes to first order such that we find the typical Jordan block and interior corners.

In passing, we note that this tangential group velocity is the main information needed in order to determine the speed of interior corners in anisotropic systems: the tip of the corner propagates with the speed given by the geometric Rankine-Hugoniot condition $c=c_{*} / \cos \vartheta$ in the normal direction of the primary flat interface, but drifts with approximately the tangential group velocity in the direction tangential to the flat interface; see Fig. 4.

Invasion of hexagons by a trivial homogeneous state, as described in [10], generates interior corners which are symmetric to an axis of symmetry of the hexagons in a similar fashion. Again, we expect corners in interfaces which are not axes of symmetry to drift in the tangential direction.

A more interesting and challenging problem arises when the group velocity of the periodic pattern is directed away from the interface. The linearization is Fredholm near the origin in exponentially weighted spaces with $\eta<0$, such that exponential growth of perturbations is allowed at $y \rightarrow+\infty$. The kernel of the linearization is two-dimensional, with space- and time- (or $x$-) derivative of the interface contributing to the kernel. We would therefore expect a four-dimensional center manifold with possibly rich dynamics as presented in Section 5. However, a rigorous reduction to a center-manifold along the lines of Section 5 fails, since nonlinearities are badly behaved in spaces of exponentially growing functions. Of course, periodic dependence of the asymptotic pattern on $x$ introduces an additional complication since the dynamics of the quadratic equations that we investigated in Section 5.2 might depend sensitively on a periodic forcing. 
The problem of corner formation in interfaces leaving a spatially periodic pattern behind is closely related to problems in crystal growth. The speed of propagation depends on the angle relative to the periodic pattern left behind. Unfortunately, a rigorous description of corners in this context seems to be out of reach for the methods we employed here.

Yet another possible direction of generalization would be the existence of defects in higher space dimension. For example, we expect to find radially symmetric interior corners in stable, planar interface propagation, adapting the methods from [57] to the present context.

Of substantial interest, in view of the mentioned applications, would be a description of corners with not necessarily small angle. The linear stability analysis of Section 3 can be generalized to large angle interior corners, when existence and spectral properties are granted. Following small amplitude interior corners, it would also be of interest to investigate possible bifurcations while following the corner to larger angles.

\section{Acknowledgements}

M. Haragus wishes to thank the School of Mathematics, University of Minnesota, for hospitality provided during the preparation of part of this paper. A. Scheel was partially supported by the NSF through grant DMS-0203301.

\section{Appendix A}

We show that the explicit solution $u_{1}$ to the steady-state Kuramoto-Sivashinsky equation is robust with respect to perturbations of the 3-dimensional differential equation (4.25). After a suitable scaling for $\zeta, \bar{\eta}_{1}$, and $\bar{c}$, Eq. (4.25) reads

$$
u^{\prime \prime \prime}=c-u^{\prime}-\frac{1}{2} u^{2}
$$

where $c>0$. The heteroclinic connection $u_{1}$ in (4.29) is found for

$$
c=2 \alpha^{2}, \quad \alpha=15 \sqrt{11 / 19^{3}}, \quad \beta=\frac{1}{2} \sqrt{11 / 19} .
$$

The linearization of (A.1) about $u_{1}$ is

$$
\mathcal{L}_{1} v=v^{\prime \prime \prime}+v^{\prime}+u_{1} v .
$$

We claim that $\mathcal{L}_{1}$ considered as a closed operator on $B C^{0}(\mathbb{R})$ is Fredholm of index -1 . The Fredholm index is readily calculated from the Morse indices of the asymptotic equilibria in the associated first-order differential equation [47,51]. Note that the unstable manifold of the equilibrium at $-\infty$ and the stable manifold of the equilibrium at $+\infty$ are both one-dimensional such that the Morse indices are 1 and 2 at $-\infty$ and $+\infty$, respectively; the Fredholm index is given by the difference of these two Morse indices, $i=-1$, as claimed above.

Next, note that the kernel of $\mathcal{L}_{1}$ is at most one-dimensional since elements provide solutions in the intersection of the tangent spaces of one-dimensional stable and unstable manifolds. The kernel is therefore spanned by $u_{1}^{\prime}$, which is an even function. We next consider the (formal) adjoint

$$
\mathcal{L}_{1}^{\mathrm{ad}} v=-v^{\prime \prime \prime}-v^{\prime}+u_{1} v
$$

The kernel of the adjoint is two-dimensional and can be decomposed into odd and even eigenfunctions. If we define the reversibility operator $R$ through $R\left(v, v^{\prime}, v^{\prime \prime}\right)=\left(-v, v^{\prime},-v^{\prime \prime}\right)$, we see that odd eigenfunctions $v$ have $\left(v, v^{\prime}, v^{\prime \prime}\right)(0) \in$ Fix $R$ and even eigenfunctions have $\left(v, v^{\prime}, v^{\prime \prime}\right)(0) \in \operatorname{Fix}(-R)$. We may restrict the eigenvalue problems for even and odd eigenfunctions to $\mathbb{R}_{+}$, say, imposing the above conditions as boundary conditions in $x=0$. The same counting arguments as above show that the restriction of $\mathcal{L}_{1}$ to the space of even functions is Fredholm 
of index 0 , and its restriction to the space of odd functions is Fredholm of index -1 . Since the kernel of $\mathcal{L}_{1}$ is one-dimensional and spanned by an even function, we conclude that the two-dimensional kernel of the adjoint is spanned by an even and an odd function.

We are now ready to show persistence of the exterior corners. First note that the reduced system (4.20)-(4.22) leads to an equation of the form

$$
u^{\prime \prime \prime}-c+u^{\prime}+\frac{1}{2} u^{2}+\varepsilon g(u)=0
$$

with an even function $g$, due to reversibility, and some small parameter $\varepsilon$. We can solve (A.2) near $\varepsilon=0, u=u_{1}$ by means of Lyapunov-Schmidt reduction on the set of odd functions, and find $u$ and $c$ as functions of $\varepsilon$ as follows.

We consider the nonlinear equation as a map from the set of odd functions in $B C^{3}(\mathbb{R}) \subset B C^{0}(\mathbb{R})$ into the set of even functions in $B C^{0}(\mathbb{R})$. The arguments above show that the linearization about $u_{1}$ is a Fredholm operator with trivial kernel and one-dimensional cokernel spanned by the even function in the kernel of $\mathcal{L}_{1}^{\text {ad }}$. However, the derivative of the left-hand side of (A.2) with respect to $c$ is given by the constant $u \equiv 1$, which is not perpendicular to cokernel with respect to our fixed $L^{2}$-scalar product. We may therefore solve the one-dimensional LyapunovSchmidt reduced equation with respect to $c$, and then find odd solutions $u=u(\varepsilon)$ of (A.2) with $c=c(\varepsilon)$ for $\varepsilon$ small.

\section{Appendix B}

We derive a model system describing transverse perturbations of the modulated planar front in the long-wave regime and determine the conditions under which the corresponding system of conservation laws (in which viscosity is neglected) has real characteristics. Recall that the modulated planar front $q_{*}\left(y, \omega_{*} t\right)$ is a solution to the reaction-diffusion system (2.1) which is $2 \pi$-periodic in the second argument. We look for perturbations of $q_{*}$ of the form

$$
u(x, y, t)=q_{*}\left(y+\xi\left(\varepsilon x, \varepsilon^{2} t\right), \omega_{*} t+\tau\left(\varepsilon x, \varepsilon^{2} t\right)\right)+\varepsilon^{2} v\left(\varepsilon x, y, \omega_{*} t, \varepsilon^{2} t\right) .
$$

Substituting (B.1) into (2.1), we find at order 0 in $\varepsilon$ Eq. (5.1) for the profile of the modulated front $q_{*}$, and at order $\varepsilon^{2}$ the equation

$$
\xi_{t} \partial_{y} q_{*}+\tau_{t} \partial_{t} q_{*}=\xi_{x x} D \partial_{y} q_{*}+\tau_{x x} D \partial_{t} q_{*}+\xi_{x}^{2} D \partial_{y y} q_{*}+2 \xi_{x} \tau_{x} D \partial_{y t} q_{*}+\tau_{x}^{2} D \partial_{t t} q_{*}-D \mathcal{L}_{*} v,
$$

in which $\mathcal{L}_{*}$ is the linear operator defined by (5.3). In order to solve this equation for $v$ in terms of $\xi$ and $\tau$ the following solvability conditions must hold

$$
\begin{aligned}
& \left\langle q_{* y}^{\mathrm{ad}}, D^{-1} \partial_{y} q_{*}\right| \xi_{t}+\left\langle q_{* y}^{\mathrm{ad}}, D^{-1} \partial_{t} q_{*}\right\rangle \tau_{t}=\xi_{x x}+\left\langle q_{* y}^{\mathrm{ad}}, \partial_{y y} q_{*}\right| \xi_{x}^{2}+\left\langle q_{* y}^{\mathrm{ad}}, \partial_{t t} q_{*}\right\rangle \tau_{x}^{2}+2\left\langle q_{* y}^{\mathrm{ad}}, \partial_{y t} q_{*}\right| \xi_{x} \tau_{x}, \\
& \left\langle q_{* t}^{\mathrm{ad}}, D^{-1} \partial_{y} q_{*}\right| \xi_{t}+\left\langle q_{* t}^{\mathrm{ad}}, D^{-1} \partial_{t} q_{*}\right\rangle \tau_{t}=\tau_{x x}+\left\langle q_{* t}^{\mathrm{ad}}, \partial_{y y} q_{*}\right| \xi_{x}^{2}+\left\langle q_{* t}^{\mathrm{ad}}, \partial_{t t} q_{*}\right\rangle \tau_{x}^{2}+2\left\langle q_{* t}^{\mathrm{ad}}, \partial_{y t} q_{*}\right) \xi_{x} \tau_{x} .
\end{aligned}
$$

Notice that the steady model system obtained in this way coincides with the reduced system (5.21)-(5.22) at $c=c_{*}$ and $\omega=\omega_{*}$ for $\eta=\xi_{x}$ and $\rho=\tau_{x}$.

Neglecting the viscosity in (B.3)-(B.4) we find

$$
\begin{aligned}
\xi_{t} & =-c_{*}\left(\frac{1}{2} \xi_{x}^{2}-\alpha_{1} \tau_{x}^{2}\right), \\
\tau_{t} & =-c_{*}\left(\xi_{x} \tau_{x}-\alpha_{2} \tau_{x}^{2}\right)
\end{aligned}
$$

in which $\alpha_{j}$ are defined as in Section 5.2,

$$
\alpha_{1}=\frac{\left\langle q_{* y}^{\mathrm{ad}}, \partial_{t t} q_{*}\right\rangle d_{22}-\left\langle q_{* t}^{\mathrm{ad}}, \partial_{t t} q_{*}\right\rangle d_{21}}{c_{*}\left(d_{11} d_{22}-d_{12} d_{21}\right)}, \quad \alpha_{2}=\frac{\left\langle q_{* t}^{\mathrm{ad}}, \partial_{t t} q_{*}\right\rangle d_{11}-\left\langle q_{* y}^{\mathrm{ad}}, \partial_{t t} q_{*}\right\rangle d_{12}}{c_{*}\left(d_{11} d_{22}-d_{12} d_{21}\right)},
$$


with

$$
\left(\begin{array}{ll}
d_{11} & d_{12} \\
d_{21} & d_{22}
\end{array}\right)=\left(\begin{array}{cc}
\left\langle q_{* y}^{\mathrm{ad}}, D^{-1} q_{* y}\right\rangle & \left\langle q_{* t}^{\mathrm{ad}}, D^{-1} q_{* y}\right\rangle \\
\left\langle q_{* y}^{\mathrm{ad}}, D^{-1} q_{* t}\right\rangle & \left\langle q_{* t}^{\mathrm{ad}}, D^{-1} q_{* t}\right\rangle
\end{array}\right) .
$$

From (B.5)-(B.6) we obtain a system of conservation laws for $\eta=\xi_{x}$ and $\rho=\tau_{x}$,

$$
\begin{aligned}
\eta_{t} & =-c_{*}\left(\eta \eta_{x}-2 \alpha_{1} \rho \rho_{x}\right), \\
\rho_{t} & =-c_{*}\left(\rho \eta_{x}+\eta \rho_{x}-2 \alpha_{2} \rho \rho_{x}\right) .
\end{aligned}
$$

A direct calculation shows that this system has real characteristics when $\alpha_{2}^{2}-2 \alpha_{1}>0$, and complex characteristics when $\alpha_{2}^{2}-2 \alpha_{1}<0$.

\section{References}

[1] J. Alexander, R. Gardner, C.K.R.T. Jones, A topological invariant arising in the stability analysis of travelling waves, J. Reine Angew. Math. 410 (1990) 167-212.

[2] M. Bardi, M.G. Crandall, L.C. Evans, H.M. Soner, P.E. Souganidis, Viscosity Solutions and Applications, Lecture Notes in Math., vol. 1660, Springer-Verlag, Berlin, 1997.

[3] A. Bonnet, F. Hamel, Existence of nonplanar solutions of a simple model of premixed Bunsen flames, SIAM J. Math. Anal. 31 (1999) 80-118.

[4] R.J. Briggs, Electron-Steam Interaction with Plasmas, MIT Press, Cambridge, 1964.

[5] V.V. Bykov, The bifurcations of separatrix contours and chaos, Physica D 62 (1993) 290-299.

[6] G. Caginalp, X. Chen, Convergence of the phase field model to its sharp interface limits, Eur. J. Appl. Math. 9 (1998) 417-445.

[7] J.W. Cahn, J. Mallet-Paret, E.S. Van Vleck, Traveling wave solutions for systems of ODEs on a two-dimensional spatial lattice, SIAM J. Appl. Math. 59 (1999) 455-493.

[8] S.R. Clarke, P.D. Miller, On the semi-classical limit for the focusing nonlinear Schrödinger equation: sensitivity to analytic properties of the initial data, Proc. Roy. Soc. London Ser. A (2002) 135-156.

[9] D.W. Davies, P. Blanchedeau, E. Dulos, P. De Kepper, Dividing blobs, chemical flowers and patterned islands in a reaction-diffusion system, J. Chem. Phys. 102 (1998) 8236-8244.

[10] A. Doelman, B. Sandstede, A. Scheel, G. Schneider, Propagation of hexagonal patterns near onset, Eur. J. Appl. Math. 14 (2003) 85-110.

[11] A. Doelman, B. Sandstede, A. Scheel, G. Schneider, The dynamics of modulated wave trains, Preprint, 2004.

[12] H. Engler, Asymptotic stability of travelling wave solutions for perturbations with algebraic decay, J. Differential Equations 185 (2002) 348-369.

[13] E. Eszter, Evans function analysis of the stability of periodic travelling wave solutions of the FitzHugh-Nagumo system, PhD Thesis, University of Massachusetts at Amherst, 1999.

[14] B. Fiedler, B. Sandstede, A. Scheel, C. Wulff, Bifurcation from relative equilibria of noncompact group actions: skew products, meanders, and drifts, Documenta Math. 1 (1996) 479-505.

[15] P.C. Fife, Dynamics of internal layers and diffusive interfaces, CBMS-NSF Regional Conf. Ser. in Appl. Math., vol. 53, SIAM, Philadelphia, PA, 1988.

[16] R.A. Gardner, K. Zumbrun, The gap lemma and geometric criteria for instability of viscous shock profiles, Comm. Pure Appl. Math. 51 (1998) 797-855.

[17] A.A. Golovin, A.A. Nepomnyashchy, B.J. Matkowsky, Traveling and spiral waves for sequential flames with translation symmetry: coupled CGL-Burgers equations, Physica D 160 (2001) 1-28.

[18] J.K. Hale, L.A. Peletier, W.C. Troy, Stability and instability in the Gray-Scott model: the case of equal diffusivities, Appl. Math. Lett. 12 (1999) 59-65.

[19] J.K. Hale, L.A. Peletier, W.C. Troy, Exact homoclinic and heteroclinic solutions of the Gray-Scott model for autocatalysis, SIAM J. Appl. Math. 61 (2000) 102-130.

[20] F. Hamel, R. Monneau, Solutions of semilinear elliptic equations in $\mathbf{R}^{N}$ with conical-shaped level sets, Comm. Partial Differential Equations 25 (2000) 769-819.

[21] F. Hamel, R. Monneau, J.-M. Roquejoffre, Stability of travelling waves in a model for conical flames in two space dimensions, Ann. Sci. École Norm. Sup. (4) 37 (2004) 469-506.

[22] C.T. Hamik, O. Steinbock, Shock structures and bunching fronts in excitable reaction-diffusion systems, Phys. Rev. E 65 (2002) 046224.

[23] M. Haragus, K. Kirchgässner, Breaking the dimension of a steady wave: some examples, in: Nonlinear Dynamics and Pattern Formation in the Natural Environment, in: Pitman Res. Notes Math. Ser., vol. 335, Longman, Harlow, 1995, pp. 119-129. 
[24] M. Haragus, K. Kirchgässner, Breaking the dimension of solitary waves, in: Progress in Partial Differential Equations: The Metz Surveys, 4, in: Pitman Res. Notes Math. Ser., vol. 345, Longman, Harlow, 1996, pp. 216-228.

[25] N. Hartmann, Y. Kevrekidis, R. Imbihl, Pattern formation in restricted geometries: the NO + CO reaction on Pt(100), J. Chem. Phys. 112 (2000) 6795-6803.

[26] S. Hastings, On the existence of homoclinic and periodic orbits for the FitzHugh-Nagumo equations, Quart. J. Math. Oxford Ser. 27 (1976) 123-134.

[27] D. Henry, Geometric Theory of Semilinear Parabolic Equations, Lecture Notes in Math., vol. 804, Springer-Verlag, New York, 1981.

[28] D. Hoff, K. Zumbrun, Asymptotic behavior of multidimensional scalar viscous shock fronts, Indiana Univ. Math. J. 49 (2000) $427-474$.

[29] T. Iibun, K. Sakamoto, Internal layers intersecting the boundary of domain in the Allen-Cahn equation, Japan J. Indust. Appl. Math. 18 (2001) 697-738.

[30] R. Imbihl, H. Engel, M. Eiswirth, Dynamics of patterns of chemical reactions on surfaces, in: Evolution of Spontaneous Structures in Dissipative Continuous Systems, in: Lecture Notes in Phys., vol. 55, Springer, Berlin, 1998, pp. 384-410.

[31] G. Iooss, M. Adelmeyer, Topics in Bifurcation Theory and Applications, second ed., Adv. Ser. Nonlinear Dynam., vol. 3, World Scientific, River Edge, NJ, 1998.

[32] C.K.R.T. Jones, Stability of the travelling wave solution of the FitzHugh-Nagumo system, Trans. Amer. Math. Soc. 286 (1984) $431-469$.

[33] C.K.R.T. Jones, R. Gardner, T. Kapitula, Stability of travelling waves for nonconvex scalar viscous conservation laws, Comm. Pure Appl. Math. 46 (1993) 505-526.

[34] M. Kærn, M. Menzinger, Pulsating wave propagation in reactive flows: flow-distributed oscillations, Phys. Rev. E 61 (2000) $3334-3338$.

[35] T. Kapitula, On the stability of travelling waves in weighted $L^{\infty}$ spaces, J. Differential Equations 112 (1994) 179-215.

[36] T. Kapitula, Multidimensional stability of planar travelling waves, Trans. Amer. Math. Soc. 349 (1997) 257-269.

[37] T. Kapitula, B. Sandstede, Stability of bright solitary wave solutions to perturbed nonlinear Schrödinger equations, Physica D 124 (1998) 58-103.

[38] P. Kent, J. Elgin, Travelling-waves of the Kuramoto-Sivashinsky equation: period-multiplying bifurcations, Nonlinearity 5 (1992) 899919.

[39] K. Kirchgässner, Wave-solutions of reversible systems and applications, J. Differential Equations 45 (1982) 113-127.

[40] J. Lega, J.V. Moloney, A.C. Newell, Swift-Hohenberg equation for lasers, Phys. Rev. Lett. 73 (1994) 2978-2981.

[41] T.-P. Liu, G. Métivier, J. Smoller, B. Temple, W.-A. Yong, K. Zumbrun, Advances in the Theory of Shock Waves, Progr. Nonlinear Differential Equations Appl., vol. 47, Birkhäuser, Boston, MA, 2001.

[42] E. Lombardi, Oscillatory Integrals and Phenomena beyond all Algebraic Orders. With Applications to Homoclinic Orbits in Reversible Systems, Lecture Notes in Math., vol. 1741, Springer-Verlag, Berlin, 2000.

[43] L. Markus, Quadratic differential equations and non-associative algebras, in: Contributions to the Theory of Nonlinear Oscillations, vol. V, Princeton University Press, Princeton, NJ, 1960, pp. 185-213.

[44] B.J. Matkowsky, D.O. Olagunju, Pulsations in a burner-stabilized premixed plane flame, SIAM J. Appl. Math. 40 (1981) $551-562$.

[45] A. Mielke, Reduction of quasilinear elliptic equations in cylindrical domains with applications, Math. Methods Appl. Sci. 10 (1988) 51-66.

[46] Y. Nishiura, D. Ueyama, Spatio-temporal chaos for the Gray-Scott model, Physica D 150 (2001) 137-162.

[47] K.J. Palmer, Exponential dichotomies and Fredholm operators, Proc. Amer. Math. Soc. 104 (1988) 149-156.

[48] L.M. Pismen, A.A. Nepomnyashchy, Propagation of the hexagonal pattern, Europhys. Lett. 27 (1994) 433-436.

[49] K. Sakamoto, Invariant manifolds in singular perturbation problems for ordinary differential equations, Proc. Roy. Soc. Edinburgh Sect. A 116 (1990) 45-78.

[50] B. Sandstede, A. Scheel, Absolute and convective instabilities of waves on unbounded and large bounded domains, Physica D 145 (2000) 233-277.

[51] B. Sandstede, A. Scheel, On the structure of spectra of modulated travelling waves, Math. Nachr. 232 (2001) 39-93.

[52] B. Sandstede, A. Scheel, Essential instabilities of fronts: bifurcation and bifurcation failure, Dynamical Systems 16 (2001) 1-28.

[53] B. Sandstede, A. Scheel, On the stability of periodic travelling waves with large spatial period, J. Differential Equations 172 (2001) 134-188.

[54] B. Sandstede, A. Scheel, Defects in oscillatory media: toward a classification, SIAM J. Appl. Dyn. Syst. 3 (2004) 1-68.

[55] B. Sandstede, A. Scheel, C. Wulff, Dynamics of spiral waves on unbounded domains using center-manifold reduction, J. Differential Equations 141 (1997) 122-149.

[56] D.H. Sattinger, On the stability of waves of nonlinear parabolic systems, Adv. in Math. 22 (1976) 312-355.

[57] A. Scheel, Radially symmetric patterns of reaction-diffusion systems, Mem. Amer. Math. Soc. 165 (2003).

[58] E. Schöll, Nonlinear Spatio-Temporal Dynamics and Chaos in Semiconductors, Cambridge Nonlinear Science Series, vol. 10, Cambridge University Press, 2001.

[59] G.I. Sivashinsky, Nonlinear analysis of hydrodynamic instability in laminar flames I. Derivation of basic equations, Acta Astronautica 4 (1977) 1177-1206.

[60] T. Tsujikawa, T. Nagai, M. Mimura, R. Kobayashi, H. Ikeda, Stability properties of traveling pulse solutions of the higher-dimensional FitzHugh-Nagumo equations, Japan J. Appl. Math. 6 (1989) 341-366.

[61] J.J. Tyson, J.P. Keener, Singular perturbation theory of traveling waves in excitable media (a review), Physica D 32 (1988) $327-361$. 
[62] W. van Saarloos, Front propagation into unstable states. II. Linear versus nonlinear marginal stability and rate of convergence, Phys. Rev. A 39 (1989) 6367-6390.

[63] W. van Saarloos, Front propagation into unstable states, Phys. Rep. 386 (2003) 29-222.

[64] A.I. Volpert, V.A. Volpert, V.A. Volpert, Traveling Wave Solutions of Parabolic Systems, Transl. Math. Monographs, vol. 140, American Mathematical Society, Providence, RI, 1994.

[65] F.A. Williams, Combustion Theory, Benjamin Cummings, Menlo Park, 1985.

[66] A.T. Winfree, The Geometry of Biological Time, second ed., Interdiscip. Appl. Math., vol. 12, Springer-Verlag, New York, 2001.

[67] E. Yanagida, Stability of fast travelling pulse solutions of the FitzHugh-Nagumo equations, J. Math. Biology 22 (1985) 81-104. 